\title{
ON PROJECTION ALGORITHMS FOR SOLVING CONVEX FEASIBILITY PROBLEMS*
}

\author{
HEINZ H. BAUSCHKE ${ }^{\dagger}$ AND JONATHAN M. BORWEIN ${ }^{\dagger}$
}

\begin{abstract}
Due to their extraordinary utility and broad applicability in many areas of classical mathematics and modern physical sciences (most notably, computerized tomography), algorithms for solving convex feasibility problems continue to receive great attention. To unify, generalize, and review some of these algorithms, a very broad and flexible framework is investigated. Several crucial new concepts which allow a systematic discussion of questions on behaviour in general Hilbert spaces and on the quality of convergence are brought out. Numerous examples are given.

Key words. angle between two subspaces, averaged mapping, Cimmino's method, computerized tomography, convex feasibility problem, convex function, convex inequalities, convex programming, convex set, Fejér monotone sequence, firmly nonexpansive mapping, Hilbert space, image recovery, iterative method, Kaczmarz's method, linear convergence, linear feasibility problem, linear inequalities, nonexpansive mapping, orthogonal projection, projection algorithm, projection method, Slater point, subdifferential, subgradient, subgradient algorithm, successive projections
\end{abstract}

AMS subject classifications. 47H09, 49M45, 65-02, 65J05, 90C25

1. Introduction, preliminaries, and notation. A very common problem in diverse areas of mathematics and physical sciences consists of trying to find a point in the intersection of convex sets. This problem is referred to as the convex feasibility problem; its precise mathematical formulation is as follows.

Suppose $X$ is a Hilbert space and $C_{1}, \ldots, C_{N}$ are closed convex subsets with nonempty intersection $C$ :

$$
C=C_{1} \cap \cdots \cap C_{N} \neq \varnothing .
$$

Convex feasibility problem: Find some point $x$ in $C$.

We distinguish two major types.

1. The set $C_{i}$ is "simple" in the sense that the projection (i.e., the nearest point mapping) onto $C_{i}$ can be calculated explicitly; $C_{i}$ might be a hyperplane or a halfspace.

2. It is not possible to obtain the projection onto $C_{i}$; however, it is at least possible to describe the projection onto some approximating superset of $C_{i}$. (There is always a trivial approximating superset of $C_{i}$, namely, $X$.) Typically, $C_{i}$ is a lower level set of some convex function.

One frequently employed approach in solving the convex feasibility problem is algorithmic. The idea is to involve the projections onto each set $C_{i}$ (resp., onto a superset of $C_{i}$ ) to generate a sequence of points that is supposed to converge to a solution of the convex feasibility problem. This is the approach we will investigate. We are aware of four distinct (although intertwining) branches, which we classify by their applications.

\section{Best approximation theory.}

Properties: Each set $C_{i}$ is a closed subspace. The algorithmic scheme is simple ("cyclic" control).

Basic results: von Neumann [103, Thm. 13.7], Halperin [61].

Comments: The generated sequence converges in norm to the point in $C$ that is closest to the starting point. Quality of convergence is well understood.

References: Deutsch [44].

${ }^{*}$ Received by the editors July 7, 1993; accepted for publication (in revised form) June 19, 1995. This research was supported by NSERC and by the Shrum Endowment.

${ }^{\dagger}$ Department of Mathematics and Statistics, Simon Fraser University, Burnaby, British Columbia, Canada V5A 1S6 (bauschke@cecm.sfu.ca and jborwein@cecm.sfu.ca). 
Areas of application: Diverse. Statistics (linear prediction theory), partial differential equations (Dirichlet problem), and complex analysis (Bergman kernels, conformal mappings), to name only a few.

II. Image reconstruction: Discrete models.

Properties: Each set $C_{i}$ is a halfspace or a hyperplane. $X$ is a Euclidean space (i.e., a finite-dimensional Hilbert space). Very flexible algorithmic schemes.

Basic results: Kaczmarz [71], Cimmino [29], Agmon [1], Motzkin and Schoenberg [83].

Comments: Behaviour in general Hilbert space and quality of convergence only partially understood.

References: Censor [21, 23, 24], Censor and Herman [27], Viergever [102], Sezan [91].

Areas of application: Medical imaging and radiation therapy treatment planning (computerized tomography), electron microscopy.

\section{Image reconstruction: Continuous models.}

Properties: $X$ is usually an infinite-dimensional Hilbert space. Fairly simple algorithmic schemes.

Basic results: Gubin, Polyak, and Raik [60].

Comments: Quality of convergence is fairly well understood.

References: Herman [63], Youla and Webb [108], Stark [95].

Areas of application: Computerized tomography, signal processing.

\section{Subgradient algorithms.}

Properties: Some sets $C_{i}$ are of type 2. Fairly simple algorithmic schemes ("cyclic" or "weighted" control).

Basic results: Eremin [52], Polyak [86], Censor and Lent [28].

Comments: Quality of convergence is fairly well understood.

References: Censor [22], Shor [92].

Areas of application: Solution of convex inequalities, minimization of convex nonsmooth functions.

To improve, unify, and review algorithms for these branches, we must study a flexible algorithmic scheme in general Hilbert space and be able to draw conclusions on the quality of convergence. This is our objective in this paper.

We will analyze algorithms in a very broad and adaptive framework that is essentially due to Flåm and Zowe [53]. (Related frameworks with somewhat different ambitions were investigated by Browder [17] and Schott [89].) The algorithmic scheme is as follows.

Given the current iterate $x^{(n)}$, the next iterate $x^{(n+1)}$ is obtained by

$$
x^{(n+1)}:=A^{(n)} x^{(n)}:=\left(\sum_{i=1}^{N} \lambda_{i}^{(n)}\left[\left(1-\alpha_{i}^{(n)}\right) \mathrm{Id}+\alpha_{i}^{(n)} P_{i}^{(n)}\right]\right) x^{(n)},
$$

where every $P_{i}^{(n)}$ is the projection onto some approximating superset $C_{i}^{(n)}$ of $C_{i}$, every $\alpha_{i}^{(n)}$ is a relaxation parameter between 0 and 2 , and the $\lambda_{i}^{(n)}$, s are nonnegative weights summing up to 1 . In short, $x^{(n+1)}$ is a weighted average of relaxed projections of $x^{(n)}$.

Censor and Herman [27] expressly suggested the study of a (slightly) restricted version of (*) in the context of computerized tomography. It is worthwhile to point out that the scheme (*) can be thought of as a combination of the schemes investigated by Aharoni, Berman, and Censor [2] and Aharoni and Censor [3]. In Euclidean spaces, norm convergence results were obtained by Flåm and Zowe for $(*)$ and by Aharoni and Censor [3] for the restricted version. However, neither behaviour in general Hilbert spaces nor quality of convergence has been much discussed so far. To do this comprehensively and clearly, it is important to bring out 
some underlying recurring concepts. We feel these concepts lie at the heart of many algorithms and will be useful for other researchers as well.

The paper is organized as follows.

In $\S 2$, the two important concepts of attracting mappings and Fejér monotone sequences are investigated. The former concept captures essential properties of the operator $A^{(n)}$, whereas the latter deals with inherent qualities of the sequence $\left(x^{(n)}\right)$.

The idea of a focusing algorithm is introduced in $\S 3$. The very broad class of focusing algorithms admits results on convergence. In addition, the well-known ideas of cyclic and weighted control are subsumed under the notion of intermittent control. Weak topology results on intermittent focusing algorithms are given. We actually study a more general form of the iteration $(*)$ without extra work; as a by-product, we obtain a recent result by Tseng [100] and make connections with work by Browder [17] and Baillon [7].

At the start of $\S 4$, we exclusively consider algorithms such as $(*)$, which we name projection algorithms. Prototypes of focusing and linearly focusing (a stronger, more quantitative version) projection algorithms are presented. When specialized to Euclidean spaces, our analysis yields basic results by Flåm and Zowe [53] and Aharoni and Censor [3].

The fifth section discusses norm and particularly linear convergence. Many known sufficient sometimes ostensibly different looking conditions for linear convergence can be thought of as special instances of a single new geometric concept-regularity. Here the $N$-tuple $\left(C_{1}, \ldots, C_{N}\right)$ is called regular if "closeness to all sets $C_{i}$ implies closeness to their intersection $C$." Four quantitative versions of (bounded) (linear) regularity are described. Having gotten all the crucial concepts together, we deduce our main results, one of which states in short that

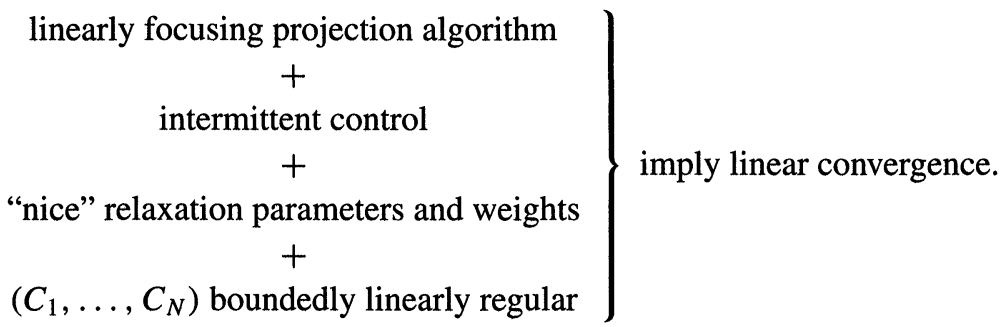

This section ends with results on (bounded) (linear) regularity, including a characterization of regular $N$-tuples of closed subspaces.

Section 6 contains a multitude of examples of algorithms from branches I, II, and III.

The final section examines the subgradient algorithms of branch IV, to which our previous results also apply. Thus, a well-known Slater point condition emerges as a sufficient condition for a subgradient algorithm to be linearly focusing, thus yielding a conceptionally simple proof of an important result by De Pierro and Iusem [40]. It is very satisfactory that analogous results are obtained for algorithms suggested by Dos Santos [47] and Yang and Murty [105].

For the reader's convenience, an index is included.

We conclude this section with a collection of frequently-used facts, definitions, and notation.

The "stage" throughout this paper is a real Hilbert space $X$; its unit ball $\{x \in X:\|x\| \leq 1\}$ is denoted $B_{X}$.

FACTS 1.1.

(i) (parallelogram law) If $x, y \in X$, then

$$
\|x+y\|^{2}+\|x-y\|^{2}=2\left(\|x\|^{2}+\|y\|^{2}\right) .
$$

(ii) (strict convexity) If $x, y \in X$, then

$$
\|x+y\|=\|x\|+\|y\| \text { implies }\|y\| \cdot x=\|x\| \cdot y .
$$

(iii) Every bounded sequence in $X$ possesses a weakly convergent subsequence. 
Proof. (i) is easy to verify and implies (ii). (iii) follows from the Eberlein-Šmulian theorem (see, for example, Holmes $[67, \S 18]$ ).

All "actors" turn out to be members of the distinguished class of nonexpansive mappings. A mapping $T: D \longrightarrow X$, where the domain $D$ is a closed convex nonempty subset of $X$, is called nonexpansive if

$$
\|T x-T y\| \leq\|x-y\| \quad \text { for all } x, y \in D \text {. }
$$

If $\|T x-T y\|=\|x-y\|$, for all $x, y \in D$, then we say $T$ is an isometry. In contrast, if $\|T x-T y\|<\|x-y\|$, for all distinct $x, y \in D$, then we speak of a strictly nonexpansive mapping. If $T$ is a nonexpansive mapping, then the set of all fixed points Fix $T$, which is defined by

Fix $T=\{x \in D: x=T x\}$,

is always closed and convex [58, Lem. 3.4].

FACT 1.2 (demiclosedness principle). If $D$ is a closed convex subset of $X, T: D \longrightarrow X$ is nonexpansive, $\left(x_{n}\right)$ is a sequence in $D$, and $x \in D$, then

$$
\left.\begin{array}{c}
x_{n} \rightarrow x \\
x_{n}-T x_{n} \rightarrow 0
\end{array}\right\} \text { implies } x \in \text { Fix } T,
$$

where, by convention, “ $\rightarrow$ " (resp., " $\rightarrow$ ") stands for norm (resp., weak) convergence.

Proof. This is a special case of Opial's [84, Lem. 2].

It is obvious that the identity Id is nonexpansive and easy to see that convex combinations of nonexpansive mappings are also nonexpansive. In particular, if $N$ is a nonexpansive mapping, then so is

$$
(1-\alpha) \operatorname{Id}+\alpha N \quad \text { for all } \alpha \in[0,1[
$$

These mappings are called averaged mappings. A firmly nonexpansive mapping is a nonexpansive mapping that can be written as

$$
\frac{1}{2} \mathrm{Id}+\frac{1}{2} N \quad \text { for some nonexpansive mapping } N .
$$

FACT 1.3. If $D$ is a closed convex subset of $X$ and $T: D \longrightarrow X$ is a mapping, then the following conditions are equivalent.

(i) $T$ is firmly nonexpansive.

(ii) $\|T x-T y\|^{2} \leq\langle T x-T y, x-y\rangle$ for all $x, y \in D$.

(iii) $2 T-$ Id is nonexpansive.

Proof. See, for example, Zarantonello's [109, §1] or Goebel and Kirk's [56, Thm. 12.1].

A mapping is called relaxed firmly nonexpansive if it can be expressed as

$$
(1-\alpha) \operatorname{Id}+\alpha F \quad \text { for some firmly nonexpansive mapping } F \text {. }
$$

COROLlaRY 1.4. Suppose $D$ is a closed convex subset of $X$ and $T: D \rightarrow X$ is a mapping. Then $T$ is averaged if and only if it is relaxed firmly nonexpansive.

The "principal actor" is the projection operator. Given a closed convex nonempty subset $C$ of $X$, the mapping that sends every point to its nearest point in $C$ (in the norm induced by the inner product of $X$ ) is called the projection onto $C$ and denoted $P_{C}$. 
FACTS 1.5. Suppose $C$ is a closed convex nonempty subset of $X$ with projection $P_{C}$. Then

(i) $P_{C}$ is firmly nonexpansive.

(ii) If $x \in X$, then $P_{C} x$ is characterized by

$$
P_{C} x \in C \quad \text { and } \quad\left\langle C-P_{C} x, x-P_{C} x\right\rangle \leq 0 .
$$

Proof. See, for example, [109, Lem. 1.2] for (i) and [109, Lem. 1.1] for (ii).

Therefore,

\author{
projection \\ $\Downarrow$ \\ firmly nonexpansive \\ $\Downarrow$ \\ relaxed firmly nonexpansive $=$ averaged \\ $\Downarrow$ \\ isometry $\Rightarrow$ nonexpansive $\Leftarrow$ strictly nonexpansive.
}

The associated function $d(\cdot, C): X \longrightarrow \mathbb{R}: x \longmapsto \inf _{c \in C}\|x-c\|=\left\|x-P_{C} x\right\|$ is called the distance function to $C$; it is easy to see that $d(\cdot, C)$ is convex and continuous (hence weakly lower semicontinuous).

A good reference on nonexpansive mappings is Goebel and Kirk's recent book [58]. Many results on projections are in Zarantonello's [109].

The algorithms' quality of convergence will be discussed in terms of linear convergence: a sequence $\left(x_{n}\right)$ in $X$ is said to converge linearly to its limit $x$ (with rate $\beta$ ) if $\beta \in[0,1[$ and there is some $\alpha \geq 0$ such that (s.t.)

$$
\left\|x_{n}-x\right\| \leq \alpha \beta^{n} \quad \text { for all } n .
$$

PROPOSITION 1.6. Suppose $\left(x_{n}\right)$ is a sequence in $X, p$ is some positive integer, and $x$ is a point in $X$. If $\left(x_{p n}\right)_{n}$ converges linearly to $x$ and $\left(\left\|x_{n}-x\right\|\right)_{n}$ is decreasing, then the entire sequence $\left(x_{n}\right)_{n}$ converges linearly to $x$.

Proof. There is some $\alpha>0$ and $\beta \in[0,1[$ s.t.

$$
\left\|x_{p n}-x\right\| \leq \alpha \beta^{n} \quad \text { for all } n .
$$

Now fix an arbitrary positive integer $m$ and divide by $p$ with remainder; i.e., write

$$
m=p \cdot n+r, \quad \text { where } r \in\{0,1, \ldots, p-1\} .
$$

We estimate

$$
\begin{aligned}
\left\|x_{m}-x\right\| & \leq\left\|x_{p n}-x\right\| \leq \alpha\left(\beta^{\frac{1}{p}}\right)^{n p} \\
& =\frac{\alpha\left(\beta^{\frac{1}{p}}\right)^{n p+r}}{\left(\beta^{\frac{1}{p}}\right)^{r}} \leq\left(\frac{\alpha}{\left(\beta^{\frac{1}{p}}\right)^{p-1}}\right)\left(\beta^{\frac{1}{p}}\right)^{m},
\end{aligned}
$$

and the result follows.

Finally, we recall the meaning of the following.

If $S$ and $Y$ are any subsets of $X$, then span $S, \overline{\operatorname{conv}} S, \bar{S}$, int $Y$, icr $S$, and int $S$ denote, respectively, the span of $S$, the closed convex hull of $S$, the closure of $S$, the interior of $S$ with 
respect to (w.r.t.) $Y$, the intrinsic core of $S\left(=\operatorname{int}_{\overline{\text { aff }} S} S\right.$, where aff $S$ is the closed affine span of $S)$, and the interior of $S\left(=\operatorname{int}_{X} S\right)$.

$S$ is called a cone if it is nonempty, convex, and closed under nonnegative scalar multiplication. If $S$ is the intersection of finitely many halfspaces, then $S$ is a polyhedron.

If $r$ is a real number, then $r^{+}:=\max \{r, 0\}$ is called the positive part of $r$. In the context of sequences of real numbers, $\varlimsup$ lim (resp., $\underline{\lim }$ ) stands for limes superior (resp., limes inferior). Occasionally, we will use the quantifiers $\forall$ (for all) and $\exists$ (there exists) to avoid monstrous sentences.

2. Two useful tools: Attracting mappings and Fejér monotone sequences. We focus on two important concepts. The first generalizes the idea of averaged (resp., strictly) nonexpansive mappings.

DEFINITION 2.1. Suppose $D$ is a closed convex nonempty set, $T: D \longrightarrow D$ is nonexpansive, and $F$ is a closed convex nonempty subset of $D$. We say that $T$ is attracting w.r.t. $F$ if for every $x \in D \backslash F, f \in F$,

$$
\|T x-f\|<\|x-f\| .
$$

In other words, every point in $F$ attracts every point outside $F$. A more quantitative and stronger version is the following.

We say that $T$ is strongly attracting w.r.t. $F$ if there is some $\kappa>0$ s.t. for every $x \in$ $D, f \in F$,

$$
\kappa\|x-T x\|^{2} \leq\|x-f\|^{2}-\|T x-f\|^{2} .
$$

Alternatively, if we want to emphasize $\kappa$ explicitly, we say that $T$ is $\kappa$-attracting w.r.t. $F$. In several instances, $F$ is Fix $T$; in this case, we simply speak of attracting, strongly attracting, or $\kappa$-attracting mappings.

REMARKS 2.2. Some authors do not require nonexpansivity in the definition of attracting mappings; see, for example, Bruck's "strictly quasi-nonexpansive mappings" [18], Elsner, Koltracht, and Neumann's "paracontracting mappings" [51], Eremin's " $F$-weakly Fejér maps" [52], and Istratescu's " $T$-mappings" [69, Chap. 6]. For our purposes, however, the above definitions are already general enough. As we will see, the class of strongly attracting mappings contains properly all averaged nonexpansive mappings and thus all relaxed projections-the mappings we are primarily interested in.

We would like to mention (but will not use) the fact that the class of attracting mappings properly contains all three of the following classes: the class of strictly nonexpansive mappings, Bruck and Reich's strongly nonexpansive mappings [19], and a very nice class of nonexpansive mappings introduced by De Pierro and Iusem [41, Def. 1]. The mapping $x \longmapsto 1-\ln \left(1+e^{x}\right)$ is a first example of a mapping that is strictly nonexpansive but not averaged; hence the class of attracting mappings is genuinely bigger than the class of strongly attracting mappings. Finally, neither class contains isometries with fixed points.

The asserted proper containment statements are demonstrated by the following example.

EXAMPLE 2.3. Suppose $D$ is a closed convex symmetric interval in $\mathbb{R}$ that contains the interval $[-1,+1]$ strictly. Let

$$
T: D \longrightarrow D: x \longmapsto\left\{\begin{array}{cc}
\frac{1}{2}|x|^{2} & \text { if }|x| \leq 1, \\
|x|-\frac{1}{2} & \text { otherwise. }
\end{array}\right.
$$

Then

- $T$ is nonexpansive and Fix $T=\{0\}$.

- $T$ is not strictly nonexpansive. 
- $T$ is not strongly nonexpansive (in the sense of Bruck and Reich [19]); in particular, $T$ is not averaged [19, Prop. 1.3].

- $T$ is not nonexpansive in the sense of De Pierro and Iusem [41, Def. 1].

- $T$ is attracting.

- $T$ is strongly attracting if and only if $D$ is compact.

LEMMA 2.4 (prototype of a strongly attracting mapping). Suppose $D$ is a closed convex nonempty set, $T: D \longrightarrow D$ is firmly nonexpansive with fixed points, and $\alpha \in] 0,2[$. Let $R:=(1-\alpha) \operatorname{Id}+\alpha T$ and $f i x x \in D, f \in$ Fix $T$. Then

(i) Fix $R=$ Fix $T$.

(ii) $\langle x-f, x-T x\rangle \geq\|x-T x\|^{2}$ and $\langle x-T x, T x-f\rangle \geq 0$.

(iii) $\|x-f\|^{2}-\|R x-f\|^{2}=2 \alpha\langle x-f, x-T x\rangle-\alpha^{2}\|x-T x\|^{2}$.

(iv) $R$ is $(2-\alpha) / \alpha$-attracting:

$$
\|x-f\|^{2}-\|R x-f\|^{2} \geq(2-\alpha) / \alpha\|x-R x\|^{2}=(2-\alpha) \alpha\|x-T x\|^{2} .
$$

Proof. (i) is immediate.

(ii): Since $T$ is firmly nonexpansive, we obtain

$$
\begin{aligned}
& \|T x-f\|^{2} \leq\langle T x-f, x-f\rangle \\
\Longleftrightarrow & \|T x-x\|^{2}+\|x-f\|^{2}+2\langle T x-x, x-f\rangle \leq\langle T x-f, x-f\rangle \\
\Longleftrightarrow & \|T x-x\|^{2} \leq\langle x-T x, x-f\rangle=\langle x-T x,(x-T x)+(T x-f)\rangle \\
\Longleftrightarrow & 0 \leq\langle x-T x, T x-f\rangle .
\end{aligned}
$$

(iii) is a direct calculation:

$$
\begin{aligned}
& \|x-f\|^{2}-\|R x-f\|^{2} \\
= & \|x-f\|^{2}-\|(1-\alpha)(x-f)+\alpha(T x-f)\|^{2} \\
= & \|x-f\|^{2}-\left[(1-\alpha)^{2}\|x-f\|^{2}+\alpha^{2}\|T x-f\|^{2}+2 \alpha(1-\alpha)\langle x-f, T x-f\rangle\right] \\
= & 2 \alpha\|x-f\|^{2}-\alpha^{2}\|x-f\|^{2}-\alpha^{2}\|T x-f\|^{2} \\
& +2 \alpha^{2}\langle x-f, T x-f\rangle-2 \alpha\langle x-f, T x-f\rangle \\
= & 2 \alpha\langle x-f,(x-f)-(T x-f)\rangle \\
& -\alpha^{2}\left[\|x-f\|^{2}+\|T x-f\|^{2}-2\langle x-f, T x-f\rangle\right] \\
= & 2 \alpha\langle x-f, x-T x\rangle-\alpha^{2}\|x-T x\|^{2} .
\end{aligned}
$$

(iv): By (ii), (iii), and the definition of $R$, we get

$$
\begin{aligned}
\|x-f\|^{2}-\|R x-f\|^{2} & =2 \alpha\langle x-f, x-T x\rangle-\alpha^{2}\|x-T x\|^{2} \\
& \geq 2 \alpha\|x-T x\|^{2}-\alpha^{2}\|x-T x\|^{2} \\
& =\alpha(2-\alpha)\|x-T x\|^{2} \\
& =(2-\alpha) / \alpha\|x-R x\|^{2} .
\end{aligned}
$$

Note that (i) and (iii) are actually true for an arbitrary nonexpansive mapping $T$; this will, however, not be needed in what follows. Since projections are firmly nonexpansive (Facts 1.5.(i)), we immediately obtain the following result which slightly improves Flåm and Zowe's [53, Lem. 1].

COROLLARY 2.5. If $P$ is the projection onto some closed convex nonempty set $S$ and $\alpha \in] 0,2[$, then $R:=(1-\alpha) \operatorname{Id}+\alpha P$ is $(2-\alpha) / \alpha$-attracting w.r.t. $S$ and for $x \in X, s \in S$,

$$
\|x-s\|^{2}-\|R x-s\|^{2} \geq(2-\alpha) \alpha d^{2}(x, S) .
$$


DEFINITION 2.6. Suppose $\left(x_{n}\right)$ is a sequence in $X$. We say that $\left(x_{n}\right)$ is asymptotically regular if

$$
x_{n}-x_{n+1} \longrightarrow 0
$$

EXAMPLE 2.7. Suppose $D$ is a closed convex nonempty set, $F$ is a closed convex nonempty subset of $D$, and $\left(T_{n}\right)_{n \geq 0}$ is a sequence of nonexpansive self mappings of $D$, where each $T_{n}$ is $\kappa_{n}$-attracting w.r.t. $F$ and $\underline{\lim }_{n} \kappa_{n}>0$. Suppose further the sequence $\left(x_{n}\right)$ is defined by

$$
x_{0} \in D \text { arbitrary, } \quad x_{n+1}:=T_{n} x_{n} \quad \text { for all } n \geq 0 .
$$

Then $\left(x_{n}\right)$ is asymptotically regular.

Proof. Fix $f \in F$ and choose $0<\kappa<\underline{\lim }_{n} \kappa_{n}$. Then for all large $n$,

$$
\kappa\left\|x_{n-1}-x_{n}\right\|^{2} \leq\left\|x_{n-1}-f\right\|^{2}-\left\|x_{n}-f\right\|^{2} .
$$

Summing these inequalities shows that $\sum_{n}\left\|x_{n-1}-x_{n}\right\|^{2}$ is finite; the result follows.

COROLLARY 2.8. Suppose $D$ is a closed convex nonempty set and $T: D \longrightarrow D$ is strongly attracting with fixed points. Then the sequence of iterates $\left(T^{n} x_{0}\right)_{n \geq 0}$ is asymptotically regular for every $x_{0} \in D$.

REMARK 2.9. The corollary is well known for firmly nonexpansive and, more generally, strongly nonexpansive mappings (see [19, Prop. 1.3 and Cor. 1.1]). In the literature, the conclusion of the corollary is often " $T$ is asymptotically regular at $x_{0}$." We hope the reader accepts this as an a posteriori justification for introducing the notion of an asymptotically regular sequence.

The next propositions show that (strongly) attracting mappings respect compositions and convex combinations.

PROPOSITION 2.10. Suppose $D$ is a closed convex nonempty set, $T_{1}, \ldots, T_{N}: D \longrightarrow D$ are attracting, and $\bigcap_{i=1}^{N}$ Fix $T_{i}$ is nonempty. Then

(i) Fix $\left(T_{N} T_{N-1} \cdots T_{1}\right)=\bigcap_{i=1}^{N}$ Fix $T_{i}$ and $T_{N} T_{N-1} \cdots T_{1}$ is attracting.

(ii) If every $T_{i}$ is $\kappa_{i}$-attracting, then $T_{N} T_{N-1} \cdots T_{1}$ is $\min \left\{\kappa_{1}, \ldots, \kappa_{N}\right\} / 2^{N-1}$-attracting.

Proof. It is enough to prove the proposition for $N=2$; the general case follows inductively.

(i): Clearly, Fix $T_{1} \cap$ Fix $T_{2} \subseteq$ Fix $\left(T_{2} T_{1}\right)$. To prove the other inclusion, pick $f \in$ Fix $\left(T_{2} T_{1}\right)$. It is enough to show that $f \in$ Fix $T_{1}$. If this were false, then $T_{1} f \notin$ Fix $T_{2}$. Now fix $\bar{f} \in$ Fix $T_{1} \cap$ Fix $T_{2}$. Then, since $T_{2}$ is attracting,

$$
\|f-\bar{f}\|=\left\|T_{2}\left(T_{1} f\right)-\bar{f}\right\|<\left\|T_{1} f-\bar{f}\right\| \leq\|f-\bar{f}\|,
$$

which is absurd. Thus Fix $T_{1} \cap$ Fix $T_{2}=$ Fix $\left(T_{2} T_{1}\right)$. It remains to show that $T_{2} T_{1}$ is attracting. Fix $x \in D \backslash$ Fix $\left(T_{2} T_{1}\right), f \in$ Fix $\left(T_{2} T_{1}\right)$. If $x=T_{1} x$, then $T_{2} x \neq x$ and hence $\left\|T_{2} T_{1} x-f\right\|=$ $\left\|T_{2} x-f\right\|<\|x-f\|$. Otherwise $x \neq T_{1} x$; then $\left\|T_{2} T_{1} x-f\right\| \leq\left\|T_{1} x-f\right\|<\|x-f\|$. In either case, $T_{2} T_{1}$ is attracting.

(ii): Given $x \in D, f \in$ Fix $\left(T_{2} T_{1}\right)$, we estimate

$$
\begin{aligned}
\left\|x-T_{2} T_{1} x\right\|^{2} \leq & \left(\left\|x-T_{1} x\right\|+\left\|T_{1} x-T_{2} T_{1} x\right\|\right)^{2} \\
\leq & 2\left(\left\|x-T_{1} x\right\|^{2}+\left\|T_{1} x-T_{2} T_{1} x\right\|^{2}\right) \\
\leq & \frac{2}{\kappa_{1}}\left(\|x-f\|^{2}-\left\|T_{1} x-f\right\|^{2}\right) \\
& \quad+\frac{2}{\kappa_{2}}\left(\left\|T_{1} x-f\right\|^{2}-\left\|T_{2} T_{1} x-f\right\|^{2}\right) \\
\leq & \frac{2}{\min \left\{\kappa_{1}, \kappa_{2}\right\}}\left(\|x-f\|^{2}-\left\|T_{2} T_{1} x-f\right\|^{2}\right) .
\end{aligned}
$$


REMARK 2.11. Let $X \supsetneqq\{0\}, D:=X, N:=2$, and $T_{1}:=T_{2}:=-$ Id. Then

Fix $T_{1} \cap$ Fix $T_{2}=\{0\} \varsubsetneqq X=$ Fix $\left(T_{2} T_{1}\right) ;$

hence the formula on the fixed point sets given in (i) of the last proposition does not hold in general for nonexpansive mappings.

PROPOSITION 2.12. Suppose $D$ is a closed convex nonempty set, $T_{1}, \ldots, T_{N}: D \longrightarrow D$ are attracting, and $\bigcap_{i=1}^{N}$ Fix $T_{i}$ is nonempty. Suppose further $\lambda_{1}, \ldots, \lambda_{N}>0$ with $\sum_{=1}^{N} \lambda_{i}=$ 1. Then

(i) Fix $\left(\sum_{i=1}^{N} \lambda_{i} T_{i}\right)=\bigcap_{i=1}^{N}$ Fix $T_{i}$ and $\sum_{i=1}^{N} \lambda_{i} T_{i}$ is attracting.

(ii) If every $T_{i}$ is $\kappa_{i}$-attracting, then $\sum_{i=1}^{N} \lambda_{i} T_{i}$ is $\min \left\{\kappa_{1}, \ldots, \kappa_{N}\right\}$-attracting.

Proof. Again we have only to consider the case when $N=2$.

(i): Once more, Fix $T_{1} \cap$ Fix $T_{2} \subseteq$ Fix $\left(\lambda_{1} T_{1}+\lambda_{2} T_{2}\right)$. Conversely, pick $f \in$ Fix $\left(\lambda_{1} T_{1}+\right.$ $\left.\lambda_{2} T_{2}\right), \bar{f} \in$ Fix $T_{1} \cap$ Fix $T_{2}$. Then

$$
\begin{aligned}
\|f-\bar{f}\| & =\left\|\lambda_{1} T_{1} f+\lambda_{2} T_{2} f-\lambda_{1} \bar{f}-\lambda_{2} \bar{f}\right\| \\
& \leq \lambda_{1}\left\|T_{1} f-\bar{f}\right\|+\lambda_{2}\left\|T_{2} f-\bar{f}\right\| \\
& \leq \lambda_{1}\|f-\bar{f}\|+\lambda_{2}\|f-\bar{f}\|=\|f-\bar{f}\| .
\end{aligned}
$$

Hence the above chain of inequalities is actually one of equalities. This, together with the strict convexity of $X$, implies $f=T_{1} f=T_{2} f$. Next, we show that $\lambda_{1} T_{1}+\lambda_{2} T_{2}$ is attracting. Suppose $x \neq \lambda_{1} T_{1} x+\lambda_{2} T_{2} x$ and $f \in$ Fix $\left(\lambda_{1} T_{1}+\lambda_{2} T_{2}\right)$. Then $x \notin$ Fix $T_{1} \cap$ Fix $T_{2}$ and thus

$$
\begin{aligned}
\left\|\lambda_{1} T_{1} x+\lambda_{2} T_{2} x-f\right\| & \leq \lambda_{1}\left\|T_{1} x-f\right\|+\lambda_{2}\left\|T_{2} x-f\right\| \\
& <\lambda_{1}\|x-f\|+\lambda_{2}\|x-f\|=\|x-f\| .
\end{aligned}
$$

(ii): If $\kappa:=\min \left\{\kappa_{1}, \kappa_{2}\right\}, x \in D$, and $f \in$ Fix $\left(\lambda_{1} T_{1}+\lambda_{2} T_{2}\right)$, then

$$
\begin{aligned}
\kappa\left\|x-\left(\lambda_{1} T_{1} x+\lambda_{2} T_{2} x\right)\right\|^{2} \leq & \kappa\left(\lambda_{1}\left\|x-T_{1} x\right\|+\lambda_{2} \| x-T_{2} x\right)^{2} \\
\leq & \kappa\left(\lambda_{1}\left\|x-T_{1} x\right\|^{2}+\lambda_{2}\left\|x-T_{2} x\right\|^{2}\right) \\
\leq & \lambda_{1} \kappa_{1}\left\|x-T_{1} x\right\|^{2}+\lambda_{2} \kappa_{2}\left\|x-T_{2} x\right\|^{2} \\
\leq & \lambda_{1}\left(\|x-f\|^{2}-\left\|T_{1} x-f\right\|^{2}\right) \\
& +\lambda_{2}\left(\|x-f\|^{2}-\left\|T_{2} x-f\right\|^{2}\right) \\
\leq & \|x-f\|^{2}-\left\|\left(\lambda_{1} T_{1} x+\lambda_{2} T_{2} x\right)-f\right\|^{2} .
\end{aligned}
$$

REMARK 2.13. In contrast to the last remark, the above proof shows that the formula Fix $\left(\sum_{i=1}^{N} \lambda_{i} T_{i}\right)=\bigcap_{i=1}^{N}$ Fix $T_{i}$ holds even if the $T_{i}$ 's are not attracting.

EXAMPLE 2.14. Suppose $S_{1}, \ldots, S_{N}$ are closed convex nonempty sets with projections $P_{1}, \ldots, P_{N}$ and with nonempty intersection. Then

$$
T:=\frac{P_{1}+P_{2} P_{1}+\cdots+P_{N} P_{N-1} \cdots P_{1}}{N}
$$

is strongly attracting, Fix $T=\bigcap_{i=1}^{N} S_{i}$, and the sequence of iterates $\left(T^{n} x_{0}\right)$ is asymptotically regular for every $x_{0}$.

The second concept captures essential properties of iterates of nonexpansive mappings.

DEFINITION 2.15. Suppose $C$ is a closed convex nonempty set and $\left(x_{n}\right)$ is a sequence in $X$. We say that $\left(x_{n}\right)_{n \geq 0}$ is Fejér monotone w.r.t. $C$ if

$$
\left\|x_{n+1}-c\right\| \leq\left\|x_{n}-c\right\| \quad \text { for all } c \in C \text { and every } n \geq 0 .
$$


THEOREM 2.16 (basic properties of Fejér monotone sequences). Suppose the sequence $\left(x_{n}\right)_{n \geq 0}$ is Fejér monotone w.r.t. C. Then

(i) $\left(x_{n}\right)$ is bounded and $d\left(x_{n+1}, C\right) \leq d\left(x_{n}, C\right)$.

(ii) $\left(x_{n}\right)$ has at most one weak cluster point in C. Consequently, $\left(x_{n}\right)$ converges weakly to some point in $C$ if and only if all weak cluster points of $\left(x_{n}\right)$ lie in $C$.

(iii) If the interior of $C$ is nonempty, then $\left(x_{n}\right)$ converges in norm.

(iv) The sequence $\left(P_{C} x_{n}\right)$ converges in norm.

(v) The following are equivalent:

1. $\left(x_{n}\right)$ converges in norm to some point in $C$.

2. $\left(x_{n}\right)$ has norm cluster points, all lying in $C$.

3. $\left(x_{n}\right)$ has norm cluster points, one lying in $C$.

4. $d\left(x_{n}, C\right) \longrightarrow 0$.

5. $x_{n}-P_{C} x_{n} \longrightarrow 0$.

Moreover, if $\left(x_{n}\right)$ converges to some $x \in C$, then $\left\|x_{n}-x\right\| \leq 2 d\left(x_{n}, C\right)$ for all $n \geq 0$.

(vi) If there is some constant $\alpha>0$ s.t. $\alpha d^{2}\left(x_{n}, C\right) \leq d^{2}\left(x_{n}, C\right)-d^{2}\left(x_{n+1}, \bar{C}\right)$ for every $n$, then $\left(x_{n}\right)$ converges linearly to some point $x$ in $C$. More precisely,

$$
\left\|x_{n}-x\right\| \leq 2(1-\alpha)^{n / 2} d\left(x_{0}, C\right) \text { for every } n \geq 0 .
$$

Proof. (i) is obvious.

(ii): For any $c \in C$, the sequence $\left(\left\|x_{n}\right\|^{2}-2\left\langle x_{n}, c\right\rangle\right)$ converges. Hence if we suppose $c_{1}, c_{2}$ are two weak cluster points of $\left(x_{n}\right)$ in $C$, then we conclude that the sequence $\left(\left\langle x_{n}, c_{1}-c_{2}\right\rangle\right)$ converges and that $\left\langle c_{1}, c_{1}-c_{2}\right\rangle=\left\langle c_{2}, c_{1}-c_{2}\right\rangle$. Thus $c_{1}=c_{2}$.

(iii): Fix $c_{0} \in$ int $C$ and get $\epsilon>0$ s.t. $c_{0}+\epsilon B_{X} \subseteq C$.

Claim: $\quad 2 \epsilon\left\|x_{n}-x_{n+1}\right\| \leq\left\|x_{n}-c_{0}\right\|^{2}-\left\|x_{n+1}-c_{0}\right\|^{2} \quad$ for all $n \geq 0$.

We can assume $x_{n} \neq x_{n+1}$. Then $c_{0}+\epsilon\left(x_{n}-x_{n+1}\right) /\left\|x_{n}-x_{n+1}\right\| \in C$ and hence, by Fejér monotonicity,

$$
\left\|\left(c_{0}+\epsilon \frac{x_{n}-x_{n+1}}{\left\|x_{n}-x_{n+1}\right\|}\right)-x_{n+1}\right\| \leq\left\|\left(c_{0}+\epsilon \frac{x_{n}-x_{n+1}}{\left\|x_{n}-x_{n+1}\right\|}\right)-x_{n}\right\| .
$$

Squaring and expanding yields the claim.

The claim implies $2 \epsilon\left\|x_{n}-x_{n+k}\right\| \leq\left\|x_{n}-c_{0}\right\|^{2}-\left\|x_{n+k}-c_{0}\right\|^{2}$ for all $n, k \geq 0$. Because the sequence $\left(\left\|x_{n}-c_{0}\right\|^{2}\right)$ converges, we recognize $\left(x_{n}\right)$ as a Cauchy sequence.

(iv): Applying the parallelogram law $\|a-b\|^{2}=2\|a\|^{2}+2\|b\|^{2}-\|a+b\|^{2}$ to $a:=$ $P_{C} x_{n+k}-x_{n+k}$ and $b:=P_{C} x_{n}-x_{n+k}$, we obtain for all $n, k \geq 0$,

$$
\begin{aligned}
\left\|P_{C} x_{n+k}-P_{C} x_{n}\right\|^{2}= & 2\left\|P_{C} x_{n+k}-x_{n+k}\right\|^{2}+2\left\|P_{C} x_{n}-x_{n+k}\right\|^{2} \\
& -4\left\|\left(P_{C} x_{n+k}+P_{C} x_{n}\right) / 2-x_{n+k}\right\|^{2} \\
\leq & 2\left\|P_{C} x_{n+k}-x_{n+k}\right\|^{2}+2\left\|P_{C} x_{n}-x_{n+k}\right\|^{2} \\
& \quad-4\left\|P_{C} x_{n+k}-x_{n+k}\right\|^{2} \\
\leq & 2\left\|P_{C} x_{n}-x_{n+k}\right\|^{2}-2\left\|P_{C} x_{n+k}-x_{n+k}\right\|^{2} \\
\leq & 2\left(\left\|P_{C} x_{n}-x_{n}\right\|^{2}-\left\|P_{C} x_{n+k}-x_{n+k}\right\|^{2}\right) .
\end{aligned}
$$

We identify $\left(P_{C} x_{n}\right)$ as a Cauchy sequence because $\left(\left\|x_{n}-P_{C} x_{n}\right\|\right)$ converges by (i). 
(v): The equivalences follow easily from (i), (iv), and the definition of Fejér monotonicity. The estimate follows from letting $k$ tend to infinity in

$$
\begin{aligned}
\left\|x_{n+k}-x_{n}\right\| & \leq\left\|x_{n+k}-P_{C} x_{n}\right\|+\left\|P_{C} x_{n}-x_{n}\right\| \\
& \leq\left\|x_{n}-P_{C} x_{n}\right\|+\left\|P_{C} x_{n}-x_{n}\right\|=2 d\left(x_{n}, C\right) .
\end{aligned}
$$

(vi): Summing the given inequalities shows that $d^{2}\left(x_{n}, C\right)$ tends to 0 ; therefore, $\left(x_{n}\right)$ converges to some point $x$ in $C$ by (v). The estimate on the rate of convergence of $\left(x_{n}\right)$ follows easily from the estimate given in (v).

REMARKS 2.17. As far as we know, the notion of Fejér monotonicity was coined by Motzkin and Schoenberg [83] in 1954. Moreau [81] inspired (iii); see also [69, Thm. 6.5.3]. (iv) rests on an idea by Baillon and Brezis [8, Lemme 3] and partially extends [46, Thm. 3.4.(c)]. Finally, (v) and (vi) appeared implicitly in Gubin, Polyak, and Raik's [60, Proof of Lem. 6].

EXAMPLE 2.18 (Krasnoselski/Mann iteration). Suppose $C$ is a closed convex nonempty set, $T: C \longrightarrow C$ is nonexpansive with fixed points, and the sequence $\left(x_{n}\right)$ is given by

$$
x_{0} \in C, \quad x_{n+1}:=\left(1-t_{n}\right) x_{n}+t_{n} T x_{n}
$$

for all $n \geq 0$ and some sequence $\left(t_{n}\right)_{n \geq 0}$ in $[0,1]$. Then $\left(x_{n}\right)$ is Fejér monotone w.r.t. Fix $T$.

REMARKS 2.19. In the early days, the Krasnoselski/Mann iteration was studied in Hilbert space. Some authors then implicitly used properties of Fejér monotone sequences; see [79, Proof of Thm. 1] and [90, Proof of Thm. 2]. However, tremendous progress has been made and today the iteration is studied in normed or even more general spaces (see $[15,57]$ for further information).

EXAMPLE 2.20 (Example 2.14 continued). The sequence $\left(T^{n} x_{0}\right)$ converges weakly to some fixed point of $T$ for every $x_{0}$.

Proof. $\left(T^{n} x_{0}\right)$ is asymptotically regular (Example 2.14) and Fejér monotone w.r.t. Fix $T$ (Example 2.18). By the demiclosedness principle, every weak limit point of $\left(T^{n} x_{0}\right)$ lies in Fix $T$. The result now follows from Theorem 2.16.(ii).

REMARK 2.21. Alternatively, one can use Baillon, Bruck, and Reich's results on averaged mappings [9, Thms. 1.2 and 2.1] to understand the last example. In fact, using a suitable modification of [9, Thm. 1.1], one can show that $\left(T^{n} x_{0}\right)$ converges in norm whenever $S_{1}, \ldots, S_{N}$ are closed affine subspaces.

REMARK 2.22. We conclude this section by mentioning a method due to Halpern [62] which generates a sequence that converges in norm to the fixed point of $T$ that is closest to the starting point. For extensions of Halpern's result, the interested reader is referred to Lions's [77], Wittmann's [104], and the first author's [11].

\section{The algorithm: Basic properties and convergence results.}

Setting. Suppose $D$ is a closed convex nonempty set and $C_{1}, \ldots, C_{N}$ are finitely many closed convex subsets of $D$ with nonempty intersection:

$$
C:=\bigcap_{i=1}^{N} C_{i} \neq \emptyset \text {. }
$$

For every $i \in\{1, \ldots, N\}$ (we will often refer to $i$ as an index) and all $n \geq 0$, suppose that $T_{i}^{(n)}: D \longrightarrow D$ is firmly nonexpansive with

$$
\text { Fix } T_{i}^{(n)} \supseteq C_{i},
$$

that $\alpha_{i}^{(n)} \in[0,2]$ is a relaxation parameter and

$$
R_{i}^{(n)}:=\left(1-\alpha_{i}^{(n)}\right) \mathrm{Id}+\alpha_{i}^{(n)} T_{i}^{(n)}
$$


is the corresponding relaxation of $T_{i}^{(n)}$ (underrelaxed if $\alpha_{i}^{(n)} \in[0,1]$, overrelaxed if $\alpha_{i}^{(n)} \in$ $[1,2])$, that $\left(\lambda_{i}^{(n)}\right)_{i=1}^{N}$ in $[0,1]$ is a weight, i.e., $\sum_{i=1}^{N} \lambda_{i}^{(n)}=1$, and finally that

$$
A^{(n)}:=\sum_{i=1}^{N} \lambda_{i}^{(n)} R_{i}^{(n)}
$$

is the corresponding weighted average of the relaxations.

With these abbreviations, we define the algorithm by the sequence

$$
x^{(0)} \in D \text { arbitrary, } \quad x^{(n+1)}:=A^{(n)} x^{(n)} \text { for all } n \geq 0,
$$

with the implicit assumption that the sequence $\left(x^{(n)}\right)$ lies in $D$. We also define the set of active indices

$$
I^{(n)}:=\left\{i \in\{1, \ldots, N\}: \lambda_{i}^{(n)}>0\right\}
$$

and we say $i$ is active at $n$ or $n$ is active for $i$ if $\lambda_{i}^{(n)}>0$; i.e., $i \in I^{(n)}$. We always assume that every index is picked infinitely often; i.e., $i$ is active at infinitely many $n$ (this is sometimes referred to as repetitive control; see [22]). To facilitate the presentation, we abbreviate

$$
\mu_{i}^{(n)}:=\lambda_{i}^{(n)} \alpha_{i}^{(n)}\left[2-\sum_{j=1}^{N} \lambda_{j}^{(n)} \alpha_{j}^{(n)}\right] \quad \text { for every index } i \text { and } n \geq 0 .
$$

For convenience, we introduce some more notions. We say the algorithm is asymptotically regular if every sequence generated by the algorithm is. We say the algorithm is unrelaxed if $\alpha_{i}^{(n)}=1$ for all $n$ and every index $i$ active at any $n$; note that in this case the algorithm reduces to a product of firmly nonexpansive mappings. We say the algorithm is singular if $I^{(n)}$ is a singleton for every $n$. Singular algorithms are also called row-action methods (see, for example, [21]). Finally, we say the algorithm is weighted if $I^{(n)}=\{1, \ldots, N\}$ for all $n$; the reader may also find the words "parallel" or "simultaneous" in the literature.

REMARK 3.1. The algorithm is a direct generalization of Flåm and Zowe's algorithm [53]. In fact, one just chooses $X$ finite dimensional and $T_{i}^{(n)}$ as the projection onto a hyperplane containing $C_{i}$. We will examine their algorithm in detail in $\S 4$.

LEMMA 3.2 (basic properties of the algorithm).

(i) If $x \in D$ and $n \geq 0$, then

$$
\begin{aligned}
\left\|x^{(n)}-x\right\|^{2}-\left\|x^{(n+1)}-x\right\|^{2}= & \sum_{i<j} \lambda_{i}^{(n)} \lambda_{j}^{(n)} \alpha_{i}^{(n)} \alpha_{j}^{(n)}\left\|T_{i}^{(n)} x^{(n)}-T_{j}^{(n)} x^{(n)}\right\|^{2} \\
& +2 \sum_{i} \lambda_{i}^{(n)} \alpha_{i}^{(n)}\left\langle x^{(n)}-T_{i}^{(n)} x^{(n)}, T_{i}^{(n)} x^{(n)}-x\right\rangle \\
& +\sum_{i} \lambda_{i}^{(n)} \alpha_{i}^{(n)}\left[2-\sum_{j} \lambda_{j}^{(n)} \alpha_{j}^{(n)}\right]\left\|x^{(n)}-T_{i}^{(n)} x^{(n)}\right\|^{2} .
\end{aligned}
$$

(ii) If $x \in \bigcap_{i \in I^{(n)}} C_{i}$ and $n \geq 0$, then

$$
\left\|x^{(n)}-x\right\|^{2}-\left\|x^{(n+1)}-x\right\|^{2} \geq \sum_{i} \mu_{i}^{(n)}\left\|x^{(n)}-T_{i}^{(n)} x^{(n)}\right\|^{2} .
$$

(iii) If $x \in \bigcap_{l=n}^{m-1} \bigcap_{i \in I^{(l)}} C_{i}$ and $m \geq n \geq 0$, then

$$
\left\|x^{(n)}-x\right\|^{2}-\left\|x^{(m)}-x\right\|^{2} \geq \sum_{l=n}^{m-1} \sum_{i} \mu_{i}^{(l)}\left\|x^{(l)}-T_{i}^{(l)} x^{(l)}\right\|^{2} .
$$

In particular, this estimate holds whenever $x \in C$. 
(iv) The sequence $\left(x^{(n)}\right)$ is Fejér monotone w.r.t. $C$ and hence is bounded. Also,

$$
+\infty>\sum_{l=0}^{+\infty} \sum_{i} \mu_{i}^{(l)}\left\|x^{(l)}-T_{i}^{(l)} x^{(l)}\right\|^{2} .
$$

(v) If $n \geq 0$, then

$$
\left\|x^{(n+1)}-x^{(n)}\right\| \leq \sum_{i} \lambda_{i}^{(n)} \alpha_{i}^{(n)}\left\|x^{(n)}-T_{i}^{(n)} x^{(n)}\right\| .
$$

Proof. (i): We omit the somewhat tedious and lengthy but elementary calculation. It is easy to see that "(i) $\Longrightarrow$ (ii) $\Longrightarrow$ (iii) $\Longrightarrow$ (iv)" (use Lemma 2.4.(ii)). (v) is immediate by the definition of the algorithm.

Using the tools from the previous section, we obtain the following corollary.

COROLLARY 3.3 (sufficient conditions for norm convergence). point in $D$.

(i) If the interior of $C$ is nonempty, then the sequence $\left(x^{(n)}\right)$ converges in norm to some

(ii) If the sequence $\left(x^{(n)}\right)$ has a subsequence $\left(x^{\left(n^{\prime}\right)}\right)$ with $d\left(x^{\left(n^{\prime}\right)}, C\right) \longrightarrow 0$, then the entire sequence $\left(x^{(n)}\right)$ converges in norm to some point in $C$.

Proof. This follows from Lemma 3.2.(iv) and Theorem 2.16.

REMARK 3.4. If the interior of $C$ is empty, then the convergence might only be weak: Genel and Lindenstrauss [56] present an example of a firmly nonexpansive self mapping $T$ of some closed convex nonempty set in $\ell_{2}$ such that the sequence of iterates $\left(T^{n} x_{0}\right)$ converges weakly but not in norm for some starting point $x_{0}$. (A norm convergent method is mentioned in Remark 2.22.) Since $\left(T^{n} x_{0}\right)$ is Fejér monotone w.r.t. Fix $T$ (Example 2.18), we conclude that Fix $T$ has empty interior and norm convergence of the algorithm needs some hypothesis.

COROLLARY 3.5 (asymptotically regular algorithms). The algorithm is asymptotically regular whenever

(i) $\underline{\lim }_{n: n \text { active for } i} \mu_{i}^{(n)}>0$ for every index $i$ or

(ii) $\varlimsup_{n: n \text { active for } i} \alpha_{i}^{(n)}<2$ for every index $i$.

Proof. (i): There exists an $\epsilon>0$ s.t. for all $n$ sufficiently large, $\mu_{i}^{(n)} \geq \epsilon$ for every index $i$ active at $n$. Lemma 3.2.(iv) implies that $\sum_{n} \sum_{i: i \text { active at } n}\left\|x^{(n)}-T_{i} x^{(n)}\right\|^{2}$ is finite. Consequently,

$$
\sum_{i: i \text { active at } n}\left\|x^{(n)}-T_{i} x^{(n)}\right\| \longrightarrow 0 .
$$

On the other hand, by Lemma 3.2.(v),

$$
\left\|x^{(n+1)}-x^{(n)}\right\| \leq \sum_{i: i \text { active at } n} \lambda_{i}^{(n)} \alpha_{i}^{(n)}\left\|x^{(n)}-T_{i} x^{(n)}\right\| .
$$

Hence $\left(x^{(n)}\right)$ is asymptotically regular.

(ii): By Lemma 2.4.(iv) and Proposition 2.12, every $A^{(n)}$ is $\kappa_{n}$-attracting w.r.t. $C$, where $\kappa_{n}:=\min \left\{\left(2-\alpha_{i}^{(n)}\right) / \alpha_{i}^{(n)}: i\right.$ active at $\left.n\right\}$. The hypothesis guarantees $\underline{\lim }_{n} \kappa_{n}>0$, so the conclusion follows from Example 2.7 .

The following simple example shows that the algorithm is not necessarily asymptotically regular.

EXAMPLE 3.6. Suppose $X:=\mathbb{R}, N:=1, T_{1}^{(n)}: \equiv P_{\{0\}}=0, \alpha_{1}^{(n)}: \equiv 2$. Then $x^{(n)}=$ $(-1)^{n} x^{(0)}$; hence the sequence $\left(x^{(n)}\right)$ is not asymptotically regular for $x^{(0)} \neq 0$.

The algorithm should converge at least weakly to some point; however, as the last example shows, further assumptions are necessary. 
DEFINITION 3.7. We say the algorithm is focusing if for every index $i$ and every subsequence $\left(x^{\left(n_{k}\right)}\right)_{k}$ of the algorithm,

$$
\left.\begin{array}{c}
x^{\left(n_{k}\right)}-x \\
x^{\left(n_{k}\right)}-T_{i}^{\left(n_{k}\right)} x^{\left(n_{k}\right)} \rightarrow 0 \\
i \text { active at } n_{k}
\end{array}\right\} \text { implies } x \in C_{i} .
$$

Thanks to the demiclosedness principle, we immediately obtain a first example.

EXAMPLE 3.8. Suppose $N:=1, T_{1}^{(n)}: \equiv T$, and $C_{1}:=$ Fix $T$. Then the algorithm is focusing.

REMARK 3.9. As almost all upcoming results show, the concept of a focusing algorithm is crucial. It can be viewed as a generalization of the demiclosedness principle for firmly nonexpansive mappings. The concept itself is investigated in Proposition 3.16 (cf. Theorem 4.3, Corollary 4.9, Theorem 7.7, and Theorem 7.12).

THEOREM 3.10 (dichotomy I). Suppose the algorithm is focusing. If $\underline{\lim }_{n: n \text { active for } i} \mu_{i}^{(n)}>$ 0 for every index $i$, then the sequence $\left(x^{(n)}\right)$ either converges in norm to some point in $C$ or has no norm cluster points at all.

Proof. In view of the Fejér monotonicity of $\left(x^{(n)}\right)$ and Theorem 2.16.(v), it suffices to show that any norm cluster point of $\left(x^{(n)}\right)$ lies in $C$. Suppose to the contrary that the theorem were false. Then there is some subsequence $\left(x^{\left(n_{k}\right)}\right)_{k}$ converging to some point $x \notin C$. Let us define

$$
I_{\text {in }}:=\left\{i \in\{1, \ldots, N\}: x \in C_{i}\right\} \text { and } I_{\text {out }}:=\left\{i \in\{1, \ldots, N\}: x \notin C_{i}\right\} ;
$$

then $I_{\text {out }}$ is nonempty. We assume, after passing to a subsequence if necessary, that

$$
I^{\left(n_{k}\right)} \cup I^{\left(n_{k}+1\right)} \cup \cdots \cup I^{\left(n_{k+1}-1\right)}=\{1, \ldots, N\} .
$$

Now get $m_{k} \in\left\{n_{k}, \ldots, n_{k+1}-1\right\}$ minimal s.t. $I^{\left(m_{k}\right)} \cap I_{\mathrm{out}} \neq \emptyset$. Thus for $n_{k} \leq m<m_{k}$, we have $I^{(m)} \subseteq I_{\text {in. }}$. Since $x \in \bigcap_{i \in I_{\text {in }}} C_{i}$, Lemma 3.2.(iii) yields $\left\|x^{\left(n_{k}\right)}-x\right\| \geq\left\|x^{\left(m_{k}\right)}-x\right\|$, which implies

$$
x^{\left(m_{k}\right)} \longrightarrow x
$$

After passing to another subsequence if necessary, we can assume that there is some index $i$ s.t.

$$
i \in I^{\left(m_{k}\right)} \cap I_{\text {out }} \quad \text { for all } k \text {. }
$$

By Lemma 3.2.(iv), $+\infty>\sum_{k} \mu_{i}^{\left(m_{k}\right)}\left\|x^{\left(m_{k}\right)}-T_{i}^{\left(m_{k}\right)} x^{\left(m_{k}\right)}\right\|^{2}$. By (2) and the hypothesis on $\left(\mu_{i}^{(n)}\right)$, we conclude that

$$
x^{\left(m_{k}\right)}-T_{i}^{\left(m_{k}\right)} x^{\left(m_{k}\right)} \longrightarrow 0 .
$$

Because the algorithm is focusing, (1), (2), and (3) imply $x \in C_{i}$, which is a contradiction to $i \in I_{\text {out }}$. Therefore, the proof is complete.

REMARKS 3.11. The finite-dimensional version of the last theorem is relatively recent and was discovered (in some form or another) independently by Flåm and Zowe [53], Tseng [100], and Elsner, Koltracht, and Neumann [51]. Unfortunately, since firmly nonexpansive mappings are not weakly continuous in general (see, for example, Zarantonello's [109, Example on p. 245]), the proof does not work in the weak topology. 
COROLlaRY 3.12. Suppose $X$ is finite dimensional and the algorithm is focusing. If $\underline{\lim }_{n: n \text { active for } i} \mu_{i}^{(n)}>0$ for every index $i$, then the sequence $\left(x^{(n)}\right)$ converges in norm to some point in $C$.

Proof. $\left(x^{(n)}\right)$ is bounded (Lemma 3.2.(iv)) and hence possesses a norm cluster point. Now apply Theorem 3.10 .

REMARK 3.13 (guaranteeing the "liminf" condition). A simple way to guarantee $\varliminf_{n: n \text { active for } i} \mu_{i}^{(n)}>0$ for some index $i$ is to assume the existence of some $\epsilon>0$ s.t.

$$
\epsilon \leq \alpha_{i}^{(n)} \leq 2-\epsilon \text { and } \epsilon \leq \lambda_{i}^{(n)} \text { for all large } n \text { active at } i,
$$

because then $\mu_{i}^{(n)} \geq \epsilon^{3}$. Moreover, this assumption is equivalent to (cf. to Corollary 3.5)

$$
\underline{\lim }_{n: n \text { active for } i} \mu_{i}^{(n)}>0 \text { and } \overline{\lim }_{n: n \text { active for } i} \alpha_{i}^{(n)}<2 .
$$

Flåm and Zowe [53] used this assumption with great success; see also Example 4.18.

EXAMPLE 3.14 (Tseng's framework [100, Thm. 1]). Suppose $X$ is finite dimensional and the algorithm is singular. Suppose further $T_{i}^{(n)}: \equiv T_{i}, C_{i}:=$ Fix $T_{i}$, and there is some $\epsilon>0$ s.t. $\epsilon \leq \alpha_{i}^{(n)} \leq 2-\epsilon$ for all $n$ and every index $i$. Then the sequence $\left(x^{(n)}\right)$ converges in norm to some point in $C$.

Proof. The demiclosedness principle readily implies that the algorithm is focusing. By the last remark, $\underline{\lim }_{n: n}$ active for $i \mu_{i}^{(n)}>0$ for every index $i$. The result follows from the last corollary.

DEFINITION 3.15. Given an algorithm, we say that $\left(T_{i}^{(n)}\right)$ converges actively pointwise to $T_{i}$ for some index $i$ if

$$
\lim _{n: n \text { active for } i} T_{i}^{(n)} d=T_{i} d \text { for every } d \in D .
$$

Proposition 3.16 (prototype of a focusing algorithm). Suppose $T_{1}, \ldots, T_{N}: D \longrightarrow D$ are firmly nonexpansive and let $C_{i}:=$ Fix $T_{i}$ for every index $i$. If $\left(T_{i}^{(n)}\right)$ converges actively pointwise to $T_{i}$ for every index $i$, then the algorithm is focusing.

Proof. Fix an index $i$ and a subsequence $\left(x^{\left(n_{k}\right)}\right)$ of $\left(x^{(n)}\right)$ with $x^{\left(n_{k}\right)} \rightarrow x \in D, x^{\left(n_{k}\right)}-$ $T_{i}^{\left(n_{k}\right)} x^{\left(n_{k}\right)} \rightarrow 0$, and $i$ active at every $n_{k}$. We must show that $x \in C_{i}$. Fix any $u \in X$. Because $T_{i}^{\left(n_{k}\right)} P_{D}$ is nonexpansive,

$$
\left\langle\left(x^{\left(n_{k}\right)}-u\right)-\left(T_{i}^{\left(n_{k}\right)} x^{\left(n_{k}\right)}-T_{i}^{\left(n_{k}\right)} P_{D} u\right), x^{\left(n_{k}\right)}-u\right\rangle \geq 0 \text { for all } k
$$

Let $k$ tend to infinity; then, by hypothesis on $\left(T_{i}^{(n)}\right)$ and $\left(x^{\left(n_{k}\right)}\right)$, we conclude that

$$
\left\langle T_{i} P_{D} u-u, x-u\right\rangle \geq 0 .
$$

Since $u$ has been chosen arbitrarily, we might as well choose $u=x+t v$, where $v$ is an arbitrary vector and $t>0$. Then

$$
\left\langle T_{i} P_{D}(x+t v)-(x+t v), v\right\rangle \leq 0
$$

hence, by letting $t$ tend to 0 , we get $\left\langle T_{i} P_{D} x-x, v\right\rangle \leq 0$. For $v=x-T_{i} P_{D} x$, we obtain

$$
x=T_{i} P_{D} x .
$$

But $x \in D$, so $P_{D} x=x$ and therefore $x \in$ Fix $T_{i}=C_{i}$. 
REMARKS 3.17.

- The last proof is a special case of an argument of Baillon [7, Chapitre 6, Démonstration du Théorème I.3].

- Note that the last proposition gives another explanation of the fact that the algorithms of Examples 3.8 and 3.14 are focusing.

DEFINITION 3.18 (control). We say the algorithm is cyclic if

$$
I^{(n-1)}=\{n \bmod N\} \quad \text { for } n \geq 1,
$$

where we use $\{1, \ldots, N\}$ as remainders. If there is a positive integer $p$ s.t.

$$
i \in I^{(n)} \cup I^{(n+1)} \cup \cdots I^{(n+p-1)} \text { for every index } i \text { and all } n \geq 0,
$$

then we speak of an intermittent or p-intermittent algorithm or of intermittent control. Following Censor [21], we call an algorithm almost cyclic if it is intermittent and singular. We say the algorithm considers only blocks and speak of block control (cf. [25]) and a block algorithm if the following two conditions hold.

1. There is a decomposition $J_{1} \cap \cdots \cap J_{M}=\{1, \ldots, N\}$ with $J_{m} \neq \emptyset$ and $J_{m} \cap J_{m^{\prime}}=\varnothing$ for all $m, m^{\prime} \in\{1, \ldots, M\}$ and $m \neq m^{\prime}$.

2. There is a positive integer $p$ s.t. for all $n \geq 0$ and every $m \in\{1, \ldots, M\}, I^{\left(n^{\prime}\right)}=J_{m}$ for some $n^{\prime} \in\{n, n+1, \ldots, n+p-1\}$.

Finally, if we want to emphasize that the active indices do not necessarily follow some form of control, then we use the phrases random control and random algorithm. Clearly,

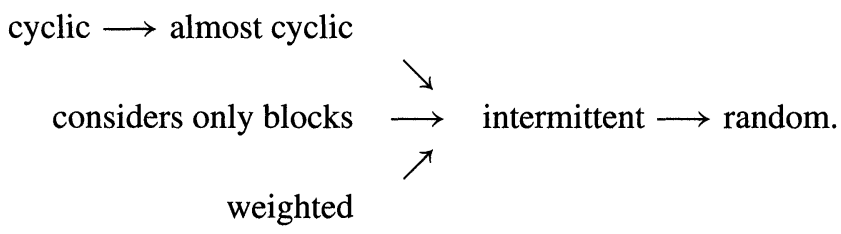

REMARKS 3.19.

- Recently, block algorithms have received much attention in radiation therapy treatment planning; see [25] and the subsection on polyhedra in $\S 6$.

- Equivalent to the phrase "almost cyclic" is Amemiya and Ando's "quasi-periodic" [5] or Browder's "admissible (for finitely many sets)" [17].

THEOREM 3.20 (weak topology results).

(i) Suppose the algorithm is focusing and intermittent. If $\underline{\lim }_{n: n \text { active for } i} \mu_{i}^{(n)}>0$ for every index $i$, then the sequence $\left(x^{(n)}\right)$ is asymptotically regular and converges weakly to some point in $C$.

(ii) Suppose the algorithm is focusing and p-intermittent for some positive integer $p$. Let

$$
v_{n}:=\min \left\{\mu_{i}^{(l)}: n p \leq l \leq(n+1) p-1 \text { and } i \text { active at } l\right\} \text { for all } n \geq 0 .
$$

If $\sum_{n} v_{n}=+\infty$, then the sequence $\left(x^{(n)}\right)$ has a unique weak cluster point in $C$. More precisely, there is a subsequence $\left(x^{\left(n_{k} p\right)}\right)$ converging weakly to this unique weak cluster point of $\left(x^{(n)}\right)$ in $C$ s.t.

$$
\sum_{l=n_{k} p}^{\left(n_{k}+1\right) p-1} \sum_{i \in I^{(l)}}\left\|x^{(l)}-T_{i}^{(l)} x^{(l)}\right\| \longrightarrow 0, \quad \text { which implies }
$$

$$
x^{\left(n_{k} p+r_{k}\right)}-x^{\left(n_{k} p+s_{k}\right)} \longrightarrow 0
$$


for all sequences $\left(r_{k}\right),\left(s_{k}\right)$ in $\{0, \ldots, p-1\}$. In particular, this happens whenever $\underline{\lim }_{n: n \text { active for } i} \mu_{i}^{(n)}>0$ for every index $i$.

(iii) Suppose the algorithm is focusing and the sequence $\left(x^{(n)}\right)$ converges weakly to some point $x$. If $\sum_{n} \mu_{i}^{(n)}=+\infty$ for some index $i$, then $x \in C_{i}$. Consequently, if $\sum_{n} \mu_{i}^{(n)}=+\infty$ for every index $i$, then $x \in C$.

Proof. (i): $\left(x^{(n)}\right)$ is asymptotically regular (Corollary 3.5.(i)). Suppose to the contrary that $\left(x^{(n)}\right)$ does not converge weakly to some point in $C$. Then, by the Fejér monotonicity of $\left(x^{(n)}\right)$ and Theorem 2.16.(ii), there exists an index $i$ and a subsequence $\left(x^{\left(n_{k}\right)}\right)_{k}$ converging weakly to some point $x \notin C_{i}$. Because the algorithm is intermittent, we obtain $m_{k}$ with

$$
n_{k} \leq m_{k} \leq n_{k}+p-1 \quad \text { and } \quad i \in I^{\left(m_{k}\right)} \text { for all } k \geq 0 .
$$

Since the algorithm is asymptotically regular, we have $x^{\left(n_{k}\right)}-x^{\left(m_{k}\right)} \longrightarrow 0$ and hence

$$
\left(x^{\left(m_{k}\right)}\right)_{k} \text { converges weakly to } x \text {. }
$$

Since the algorithm is focusing, we conclude that

$$
\underline{\lim }_{k}\left\|x^{\left(m_{k}\right)}-T_{i}^{\left(m_{k}\right)} x^{\left(m_{k}\right)}\right\|>0
$$

On the other hand, by Lemma 3.2.(iv), $+\infty>\sum_{k} \mu_{i}^{\left(m_{k}\right)}\left\|x^{\left(m_{k}\right)}-T_{i}^{\left(m_{k}\right)} x^{\left(m_{k}\right)}\right\|^{2}$. This contradicts the hypothesis on $\left(\mu_{i}^{(n)}\right)$; thus (i) holds.

(ii): Fix momentarily $c \in C$. Then, by Lemma 3.2.(iii) and the definition of $v_{n}$,

$$
\left\|x^{(n p)}-c\right\|^{2}-\left\|x^{((n+1) p)}-c\right\|^{2} \geq v_{n} \sum_{l=n p}^{(n+1) p-1} \sum_{i \in I^{(l)}}\left\|x^{(l)}-T_{i}^{(l)} x^{(l)}\right\|^{2}
$$

for all $n \geq 0$. Summing over $n$ and considering the hypothesis on $\left(v_{n}\right)$, we obtain a subsequence $\left(x^{\left(n_{k} p\right)}\right)_{k}$ s.t.

$$
\sum_{l=n_{k} p}^{\left(n_{k}+1\right) p-1} \sum_{i \in I^{(l)}}\left\|x^{(l)}-T_{i}^{(l)} x^{(l)}\right\|^{2} \longrightarrow 0 .
$$

By Lemma 3.2.(v), we also have

$$
x^{\left(n_{k} p+r_{k}\right)}-x^{\left(n_{k} p+s_{k}\right)} \longrightarrow 0
$$

for all sequences $\left(r_{k}\right),\left(s_{k}\right)$ in $\{0,1, \ldots, p-1\}$. After passing to a subsequence if necessary, we may assume that $\left(x^{\left(n_{k} p\right)}\right)_{k}$ converges weakly to some $x \in D$.

Claim:

$$
x \in C \text {. }
$$

Fix any index $i$. Since the algorithm is intermittent, there is some sequence $\left(r_{k}\right)$ in $\{0,1, \ldots, p-1\}$ s.t.

$$
x^{\left(n_{k} p+r_{k}\right)} \rightarrow x
$$

(this follows from $(* *)$ with $s_{k} \equiv 0$ ) and

$$
i \in I^{\left(n_{k} p+r_{k}\right)} \quad \text { for all } k \text {. }
$$


By $(*)$,

$$
x^{\left(n_{k} p+r_{k}\right)}-T_{i}^{\left(n_{k} p+r_{k}\right)} x^{\left(n_{k} p+r_{k}\right)} \longrightarrow 0 .
$$

Since the algorithm is focusing, (1), (2), and (3) imply $x \in C_{i}$. The claim follows.

By Theorem 2.16.(ii), $x$ is the unique weak cluster point of $\left(x^{(n)}\right)$ in $C$. The proof of (ii) is complete.

(iii): By Lemma 3.2.(iv), $+\infty>\sum_{n} \mu_{i}^{(n)}\left\|x^{(n)}-T_{i}^{(n)} x^{(n)}\right\|^{2}$. Since we assume $\sum_{n} \mu_{i}^{(n)}=$ $+\infty$, the limes inferior of the sequence

$$
\left(\left\|x^{(n)}-T_{i}^{(n)} x^{(n)}\right\|\right)_{n: n \text { active for } i}
$$

must equal 0 . Since the algorithm is focusing, we readily see that $x$ is in $C_{i}$. The entire theorem is proven.

REMARKS 3.21. (i) is our basic weak convergence result. (ii) is a generalization of an idea due to Trummer [97, Thm. 1]. Tseng's [100, Thm. 2] is also a result on the existence of a unique weak cluster point of $\left(x^{(n)}\right)$ in $C$; his hypothesis, however, is somewhat contrasting: he considers less general relaxation parameters and weights but more general control. (iii) can be viewed as a generalization of Flåm and Zowe's [53, Thm. 2] (see also Corollary 3.24 and Example 4.18.(ii)) and Aharoni and Censor's [3, Thm. 1] (see also Corollary 4.17 and Example 4.19).

CoROllary 3.22. Suppose $T_{1}, \ldots, T_{N}: D \rightarrow D$ are firmly nonexpansive, $C_{i}:=$ Fix $T_{i}$, and $\left(T_{i}^{(n)}\right)$ converges actively pointwise to $T_{i}$. Suppose further there is some $\epsilon>0$ s.t. $\epsilon \leq \alpha_{i}^{(n)} \leq 2-\epsilon$ and $\epsilon \leq \lambda_{i}^{(n)}$ for all $n \geq 0$ and every index $i$ active at $n$. If the algorithm is intermittent, then the sequence $\left(x^{(n)}\right)$ converges weakly to some point in $C$.

Proof. The algorithm is focusing (Proposition 3.16) and $\underline{\lim }_{n: n \text { active for } i} \mu_{i}^{(n)}>0$ for every index $i$ (Remark 3.13). The result now follows from Theorem 3.20.(i).

REMARKS 3.23.

(i) (a special case of a theorem of Browder) If the algorithm is almost cyclic and $T_{i}^{(n)} \equiv T_{i}$, then the last corollary gives [17, Thm. 5 for finitely many sets].

(ii) (a remark of Baillon) If the algorithm is almost cyclic and unrelaxed, then the last corollary gives Baillon's [7, Chapitre 6, Remarque II.2].

COROLLARY 3.24. Suppose the algorithm is focusing and the interior of $C$ is nonempty. If $\sum_{n} \mu_{i}^{(n)}=+\infty$ for every index $i$, then the sequence $\left(x^{(n)}\right)$ converges in norm to some point in $C$.

Proof. It is immediate from Corollary 3.3.(i) and Theorem 3.20.(iii).

COROLlARY 3.25. Suppose $X$ is finite dimensional and the algorithm is focusing and $p$ intermittent. If $\sum_{n} v_{n}=+\infty$ (where $v_{n}$ is defined as in Theorem 3.20.(ii)), then the sequence $\left(x^{(n)}\right)$ converges in norm to some point in $C$.

Proof. By Theorem 3.20.(ii), $\left(x^{(n)}\right)$ has a weak cluster point $x \in C$. Since $X$ is finite dimensional, the point $x$ is a norm cluster point of $\left(x^{(n)}\right)$. Now apply Corollary 3.3.(ii).

REMARK 3.26 (guaranteeing the "divergent sum" condition). One way to guarantee $\sum_{n} \mu_{i}^{(n)}=+\infty$ for some index $i$ is to assume that there exists some $\epsilon>0$ s.t.

$$
\epsilon \leq \alpha_{i}^{(n)} \leq 2-\epsilon \text { for all } n \text { and } \sum_{n} \lambda_{i}^{(n)}=+\infty
$$

This corresponds (in the case when the $T_{i}$ 's are projections) to Flåm and Zowe's [53, Thm. 2] (see also Example 4.18.(ii)). Another way is to assume that

$$
\text { the algorithm is singular and } \sum_{n: n \text { active for } i} \alpha_{i}^{(n)}\left(2-\alpha_{i}^{(n)}\right)=+\infty,
$$

because then the preceding sum equals $\sum_{n} \mu_{i}^{(n)}$. 
4. Harvest time I: Projection algorithms. From now on, we consider exclusively the following situation.

Setting. We maintain the hypotheses of the last section, where we defined the algorithm. In addition, we assume that $T_{i}^{(n)}$ is the projection onto some closed convex nonempty set $C_{i}^{(n)}$ containing $C_{i}$ :

$$
T_{i}^{(n)}:=P_{i}^{(n)}:=P_{C_{i}^{(n)}} \text { and } C_{i}^{(n)} \supseteq C_{i} \text { for every index } i \text { and all } n \geq 0 .
$$

We also assume that $D:=X$; that is possible since projections are defined everywhere. We abbreviate

$$
P_{i}:=P_{C_{i}} \text { for every index } i \in\{1, \ldots, N\}
$$

and refer to the algorithm in this setting as the projection algorithm. We say the projection algorithm has constant sets if $C_{i}^{(n)} \equiv C_{i}$ for all $n \geq 0$ and every index $i$.

REMARK 4.1. The projection algorithm is formally a slight generalization of Flåm and Zowe's algorithm [53] (cf. Remark 3.1). Nevertheless, since we allow infinite-dimensional Hilbert spaces and assume less restrictive hypotheses, we will obtain a number of genuinely more general results.

Of course, all the results of the previous section may be applied to the projection algorithm. However, before we can do so, we first must understand the meaning of a focusing projection algorithm. A first prototype is formulated in terms of set convergence in the sense of Mosco [82] (see [10] for a good survey article on set convergence). It is essentially a reformulation of Tsukada's [101] characterization of Mosco convergence.

LEMMA 4.2. Suppose $\left(S_{n}\right)$ is a sequence of closed convex sets and there is some closed convex nonempty set $S$ with $S \subseteq S_{n}$ for all $n$. Then the following conditions are equivalent.

(i) $P_{S_{n}} \longrightarrow P_{S}$ pointwise in norm.

(ii) $S_{n} \longrightarrow S$ in the sense of Mosco; i.e., the following two conditions are satisfied.

(a) For every $s \in S$, there exists a sequence $\left(s_{n}\right)$ converging in norm to $s$ with $s_{n} \in S_{n}$ for all $n$.

(b) If $\left(s_{n_{k}}\right)_{k}$ is a weakly convergent sequence with $s_{n_{k}} \in S_{n_{k}}$ for all $k$, then its weak limit lies in $S$.

(iii) If $\left(x_{n_{k}}\right)_{k}$ is a weakly convergent sequence with $x_{n_{k}}-P_{S_{n_{k}}} x_{n_{k}} \longrightarrow 0$, then its weak limit lies in $S$.

Moreover, if one (and hence each) of the above conditions is satisfied, then

$$
S=\bigcap_{n} S_{n}
$$

Proof. "(i) $\Longleftrightarrow$ (ii)": This is the Hilbert space case of Tsukada's [101, Thm. 3.2]. The proof of "(ii) $\Longleftrightarrow$ (iii)" and the "Moreover" part is easy and is thus omitted.

THEOREM 4.3 (first prototype of a focusing projection algorithm). If $\left(P_{i}^{(n)}\right)$ converges actively pointwise to $P_{i}$ for every index $i$, then the projection algorithm is focusing and $C_{i}=\bigcap_{n: n \text { active for } i} C_{i}^{(n)}$ for every index $i$.

Proof. Apply Lemma 4.2 to $\left(C_{i}^{(n)}\right)_{n: n \text { active for } i}$ for every index $i$.

EXAMPLE 4.4. Suppose $C_{i}=\bigcap_{n} C_{i}^{(n)}$ and $\left(C_{i}^{(n)}\right)_{n}$ is decreasing; i.e.,

$$
C_{i}^{(1)} \supseteq C_{i}^{(2)} \supseteq \cdots \supseteq C_{i}^{(n)} \supseteq C_{i}^{(n+1)} \supseteq \cdots \quad \text { for all } n \geq 0 \text { and every index } i .
$$

Then the projection algorithm is focusing. If, furthermore, the projection algorithm is intermittent and $\varliminf_{n: n \text { active for } i} \mu_{i}^{(n)}>0$ for every index $i$, then the sequence $\left(x^{(n)}\right)$ is asymptotically regular and converges weakly to some point in $C$. 
Proof. Mosco proved that a decreasing sequence of closed convex sets converges to its intersection in his sense [82, Lem. 1.3]. The last theorem and the last lemma imply that the projection algorithm is focusing. The result now follows from Theorem 3.20.(i).

REMARK 4.5. Baillon obtained the last example when the algorithm is in addition almost cyclic and unrelaxed [7, Chapitre 6, Remarque II.6].

EXAMPLE 4.6 (random projections). Suppose the projection algorithm is singular, unrelaxed, and has constant sets. If for some index $j$ the set $C_{j}$ is boundedly compact, then the sequence $\left(x^{(n)}\right)$ converges in norm to some point in $C$. In particular, this holds whenever $X$ is finite dimensional.

Proof. The last example shows that the algorithm is focusing. Also, $\mu_{i}^{(n)}=1$ for all $n \geq 0$ and every index $i$ active at $n$. The sequence $\left(x^{(n)}\right)_{n: n \text { active for } j}$ lies in $C_{j}$ and thus must have a norm cluster point; therefore, by Theorem 3.10 , the entire sequence $\left(x^{(n)}\right)$ converges in norm to some point in $C$.

REMARKS 4.7. The finite-dimensional version of the last example also follows from Aharoni and Censor's [3, Thm. 1], Flåm and Zowe's [53, Thm. 1], Tseng's [100, Thm. 1], and Elsner, Koltracht, and Neumann's [51, Thm. 1]. We discuss generalizations of Example 4.6 in $\S 6$. Not too much is known when the compactness assumption is dropped. It is known that weak convergence is obtained whenever

(i) $N=2$ or

(ii) each $C_{i}$ is a subspace,

but no example is known where the convergence is not actually in norm.

Case (i) is also known as von Neumann's alternating projection algorithm. Since projections are idempotent, one can view the sequence generated by the random projection algorithm as an alternating projection algorithm. In [13], we discussed this algorithm in some detail and provided sufficient conditions for norm (or even linear) convergence.

In 1965, Amemiya and Ando [5] proved weak convergence for Case (ii)-this is still one of the best results. Recently, the first author [12] obtained norm convergence for Case (ii) whenever a certain condition (which holds, for example, if all subspaces have finite codimension) is satisfied.

In order to formulate the second prototype of a focusing projection algorithm (as well as the norm and linear convergence results in the following sections), we require some more definitions.

DEFINITION 4.8. We say a projection algorithm is linearly focusing if there is some $\beta>0$ s.t.

$$
\beta d\left(x^{(n)}, C_{i}\right) \leq d\left(x^{(n)}, C_{i}^{(n)}\right) \text { for all large } n \text { and every index } i \text { active at } n .
$$

We speak of a strongly focusing projection algorithm if

$$
\left.\begin{array}{c}
x^{\left(n_{k}\right)}-x \\
d\left(x^{\left(n_{k}\right)}, C_{i}^{\left(n_{k}\right)}\right) \rightarrow 0 \\
i \text { active at } n_{k}
\end{array}\right\} \text { implies } d\left(x^{\left(n_{k}\right)}, C_{i}\right) \rightarrow 0
$$

for every index $i$ and every subsequence $\left(x^{\left(n_{k}\right)}\right)_{k}$ of $\left(x^{(n)}\right)$.

By Definition 3.7 and the weak lower semicontinuity of $d\left(\cdot, C_{i}\right)$, we obtain the following: linearly focusing $\Longrightarrow$ strongly focusing $\Longrightarrow$ focusing.

COROLLARY 4.9 (second prototype of a focusing projection algorithm). Every linearly focusing projection algorithm is focusing.

REMARK 4.10. Flåm and Zowe [53] used linearly focusing projection algorithms in Euclidean spaces with great success (see also Example 4.18). 
COROLLARY 4.11 (prototype of a linearly focusing projection algorithm). If the projection algorithm has constant sets, then it is linearly focusing.

COROLLARY 4.12 (prototype of a strongly focusing projection algorithm). Suppose the projection algorithm is focusing. If the terms of the sequence $\left(x^{(n)}\right)$ form a relatively compact set, then the projection algorithm is strongly focusing. In particular, this happens whenever $X$ is finite dimensional or the interior of $C$ is nonempty.

Proof. Suppose not. Then we get $\epsilon>0, x \in X$, an index $i$, and a subsequence $\left(x^{\left(n_{k}\right)}\right)_{k}$ with $x^{\left(n_{k}\right)} \rightarrow x, x^{\left(n_{k}\right)}-P_{i}^{\left(n_{k}\right)} x^{\left(n_{k}\right)} \rightarrow 0, i$ active at $n_{k}$, but $\left\|x^{\left(n_{k}\right)}-P_{i} x^{\left(n_{k}\right)}\right\| \geq \epsilon$ for all $k$. Since the algorithm is focusing, $x \in C_{i}$. After passing to a subsequence if necessary, we may assume (by the compactness assumption) that $x^{\left(n_{k}\right)} \rightarrow x$. But then $x^{\left(n_{k}\right)}-P_{i} x^{\left(n_{k}\right)} \rightarrow x-P_{i} x=0$, which is absurd. Therefore, the projection algorithm is strongly focusing. If $X$ is finite dimensional, then the terms of $\left(x^{(n)}\right)$ form a relatively compact set because $\left(x^{(n)}\right)$ is bounded (Lemma 3.2.(iv)). Finally, if int $C \neq \emptyset$, then $\left(x^{(n)}\right)$ converges in norm (Corollary 3.3.(i)). The proof is complete.

The two prototypes of a focusing projection algorithm (cf. Theorem 4.3 and Corollary 4.9) are unrelated, as the following examples demonstrate.

EXAMPLE 4.13. Suppose $X:=\mathbb{R}, N:=1, C:=C_{1}:=\{0\}, C_{1}^{(n)}:=[0,1 /(n+1)]$, and $x^{(0)}:=2$. Then the projection algorithm is strongly focusing and the sequence $\left(C_{1}^{(n)}\right)$ of compact convex decreasing sets converges to $C_{1}$ in the sense of Mosco (Example 4.4 and Corollary 4.12). However, the projection algorithm is not linearly focusing. Indeed, for $n \geq 1$,

$$
x^{(n)}=\frac{1}{n} \text { and } \quad \frac{d\left(x^{(n)}, C_{1}^{(n)}\right)}{d\left(x^{(n)}, C_{1}\right)}=\frac{1}{n+1} \longrightarrow 0 .
$$

EXAMPLE 4.14. Suppose $X:=\mathbb{R}, N:=1, C:=C_{1}:=\{0\}, C_{1}^{(n)}:=(-1)^{n}[0,1]$, and $x^{(0)} \in X$ arbitrary. Then the projection algorithm is linearly focusing since $x^{(n)} \equiv 0 \in C$ for $n \geq 2$. However, the sequence $\left(C_{1}^{(n)}\right)$ of compact convex sets does not converge to $C$ in the sense of Mosco.

Having gotten a feeling for the concept of a linearly focusing projection algorithm, we document its usefulness through a dichotomy result inspired by Aharoni and Censor's [3, Proof of Thm. 1].

THEOREM 4.15 (dichotomy II). Suppose the projection algorithm is linearly focusing and there is some $\epsilon>0$ s.t. $\epsilon \leq \alpha_{i}^{(n)} \leq 2-\epsilon$ for all large $n$ and every index $i$ active at $n$. Then the sequence $\left(x^{(n)}\right)$ either converges in norm or has no norm cluster points at all.

Proof. Assume to the contrary that $\left(x^{(n)}\right)$ has at least two distinct norm cluster points, say $y$ and $z$. Get $\beta>0$ s.t. $\beta d\left(x^{(l)}, C_{i}\right) \leq d\left(x^{(l)}, C_{i}^{(l)}\right)$ for all large $l$ and every index $i$ active at $l$. Fix $c \in C$. Since $y \notin C$ (otherwise, the sequence $\left(x^{(n)}\right)$ would converge in norm by Corollary 3.3.(ii)), the set of indices $I:=\left\{i \in\{1, \ldots, N\}: y \notin C_{i}\right\}$ is nonempty. Define $B:=y+r B_{X}$, where $r:=(1 / 2) \min \left(\{\|y-z\|\} \cup\left\{d\left(y, C_{i}\right): i \in I\right\}\right)$.

Claim 1: $\left.\quad \begin{array}{ll}\exists & x^{(l)} \in B \\ \gamma_{1}>0 & l \text { large }\end{array}\right\}$ implies $\left\|x^{(l)}-c\right\|-\left\|x^{(l+1)}-c\right\| \geq \gamma_{1} \sum_{i \in I} \lambda_{i}^{(l)}$.

On the one hand, by Lemma 3.2.(ii), the definition of $\beta$, and $\left\|y-x^{(l)}\right\| \geq d\left(y, C_{i}\right)-$ $d\left(x^{(l)}, C_{i}\right)$,

$$
\begin{aligned}
\left\|x^{(l)}-c\right\|^{2}-\left\|x^{(l+1)}-c\right\|^{2} & \geq \sum_{i \in I} \mu_{i}^{(l)} d^{2}\left(x^{(l)}, C_{i}^{(l)}\right) \\
& \geq \sum_{i \in I} \lambda_{i}^{(l)} \epsilon^{2} \beta^{2} d^{2}\left(x^{(l)}, C_{i}\right) \\
& \geq \epsilon^{2} \beta^{2} r^{2} \sum_{i \in I} \lambda_{i}^{(l)} .
\end{aligned}
$$


On the other hand,

$$
\left\|x^{(l)}-c\right\|^{2}-\left\|x^{(l+1)}-c\right\|^{2}=\left(\left\|x^{(l)}-c\right\|-\left\|x^{(l+1)}-c\right\|\right) \times\left(\left\|x^{(l)}-c\right\|+\left\|x^{(l+1)}-c\right\|\right),
$$

and the norm of the latter factor is at most $2(r+\|y-c\|)$. Altogether, $\gamma_{1}=\epsilon^{2} \beta^{2} r^{2} /(2(r+$ $\|y-c\|))$ does the job and Claim 1 is verified.

Claim 2:

$$
\left.\begin{array}{cc}
\exists & x^{(l)} \in B \\
\gamma_{2}>0 & l \text { large }
\end{array}\right\} \text { implies }\left\|x^{(l+1)}-y\right\|-\left\|x^{(l)}-y\right\| \leq \gamma_{2} \sum_{i \in I} \lambda_{i}^{(l)} .
$$

For every $i \in\{1, \ldots, N\} \backslash I$, the point $y$ is fixed under the nonexpansive mapping $R_{i}^{(l)}$ (cf. Facts 1.5.(i), Fact 1.3.(iii), and Corollary 1.4); thus we estimate

$$
\begin{aligned}
\left\|x^{(l+1)}-y\right\| & =\left\|\sum_{i \in\{1, \ldots, N\} \backslash I} \lambda_{i}^{(l)}\left(R_{i}^{(l)} x^{(l)}-y\right)+\sum_{i \in I} \lambda_{i}^{(l)}\left(R_{i}^{(l)} x^{(l)}-y\right)\right\| \\
& \leq \sum_{i \in\{1, \ldots, N\} \backslash I} \lambda_{i}^{(l)}\left\|x^{(l)}-y\right\|+\sum_{i \in I} \lambda_{i}^{(l)}\left\|R_{i}^{(l)} x^{(l)}-y\right\| \\
& \leq\left\|x^{(l)}-y\right\|+\sum_{i \in I} \lambda_{i}^{(l)}\left\{\left\|R_{i}^{(l)} x^{(l)}-x^{(l)}\right\|+\left\|x^{(l)}-y\right\|\right\} \\
& \leq\left\|x^{(l)}-y\right\|+\sum_{i \in I} \lambda_{i}^{(l)}\left\{\alpha_{i}^{(l)}\left\|x^{(l)}-P_{i}^{(l)} x^{(l)}\right\|+\left\|x^{(l)}-y\right\|\right\} \\
& \leq\left\|x^{(l)}-y\right\|+\sum_{i \in I} \lambda_{i}^{(l)}\left\{2 d\left(x^{(l)}, C_{i}\right)+r\right\} \\
& \leq\left\|x^{(l)}-y\right\|+\sum_{i \in I} \lambda_{i}^{(l)}\left\{2\left(d\left(y, C_{i}\right)+\left\|x^{(l)}-y\right\|\right)+r\right\} .
\end{aligned}
$$

Therefore, $\gamma_{2}=2 \max \left\{d\left(y, C_{i}\right): i \in I\right\}+3 r$ does the job and Claim 2 is also verified.

The rest is done quickly. Set

$$
\delta:=r \frac{\gamma_{1}}{\gamma_{1}+\gamma_{2}} \quad(<r)
$$

and find $n$ large s.t. $\left\|x^{(n)}-y\right\|<\delta$; thus $x^{(n)} \in B$. Now $z$ is another norm cluster point of $\left(x^{(n)}\right)$ and has positive distance to $B$, so there is a minimal $m>n$ with $x^{(m)} \notin B$. By the Fejér monotonicity of $\left(x^{(n)}\right)$ and Claim 1 ,

$$
\begin{aligned}
\|y-c\| & \leq\left\|x^{(m)}-c\right\| \leq\left\|x^{(n)}-c\right\|-\gamma_{1} \sum_{l=n}^{m-1} \sum_{i \in I} \lambda_{i}^{(l)} \\
& <\delta+\|y-c\|-\gamma_{1} \sum_{l=n}^{m-1} \sum_{i \in I} \lambda_{i}^{(l)}
\end{aligned}
$$

thus

$$
\sum_{l=n}^{m-1} \sum_{i \in I} \lambda_{i}^{(l)}<\frac{\delta}{\gamma_{1}}
$$

By Claim 2, however,

$$
\left\|x^{(m)}-y\right\| \leq\left\|x^{(n)}-y\right\|+\gamma_{2} \sum_{l=n}^{m-1} \sum_{i \in I} \lambda_{i}^{(l)}<\delta+\frac{\gamma_{2}}{\gamma_{1}} \delta=r,
$$


which contradicts $y \notin B$. Therefore, the sequence $\left(x^{(n)}\right)$ has at most one norm cluster point.

REMARK 4.16. As Example 3.6 demonstrates, some assumption is necessary to guarantee at most one norm cluster point.

COROLLARY 4.17. Suppose the projection algorithm is linearly focusing and there is some $\epsilon>0$ s.t. $\epsilon \leq \alpha_{i}^{(n)} \leq 2-\epsilon$ for all large $n$ and every index $i$ active at $n$. Suppose further that $X$ is finite dimensional or the interior of $C$ is nonempty. Then the sequence $\left(x^{(n)}\right)$ converges in norm to some point $x$. If $\sum_{n} \mu_{i}^{(n)}=+\infty$ for some index $i$, then $x \in C_{i}$. Consequently, if $\sum_{n} \mu_{i}^{(n)}=+\infty$ for every index $i$, then $x \in C$.

Proof. If int $C \neq \emptyset$, then $\left(x^{(n)}\right)$ converges in norm by Corollary 3.3.(i). If $X$ is finite dimensional, then $\left(x^{(n)}\right)$ has a norm cluster point; thus, by the last theorem, $\left(x^{(n)}\right)$ is also norm convergent. The result now follows from Theorem 3.20.(iii).

The next two examples follow immediately.

EXAMPLE 4.18 (Flåm and Zowe's framework [53, Thms. 1 and 2]). Suppose $X$ is finite dimensional, the projection algorithm is linearly focusing, and there is some $\epsilon>0$ s.t. $\epsilon \leq$ $\alpha_{i}^{(n)} \leq 2-\epsilon$ for all large $n$ and every index $i$ active at $n$. Then the sequence $\left(x^{(n)}\right)$ converges in norm to some point $x$.

(i) If $\underline{\lim }_{n: n \text { active for } i} \mu_{i}^{(n)}>0$ for every index $i$, then $x \in C$.

(ii) If int $C \neq \varnothing$ and $\sum_{n} \mu_{i}^{(n)}=+\infty$ for every index $i$, then $x \in C$.

EXAMPLE 4.19 (Aharoni and Censor's framework [3, Thm. 1]). Suppose $X$ is finite dimensional, the projection algorithm has constant sets (and is therefore linearly focusing by Corollary 4.11), and there is some $\epsilon>0$ s.t. $\epsilon \leq \alpha_{i}^{(n)} \equiv \alpha^{(n)} \leq 2-\epsilon$ for all large $n$ and every index $i$ active at $n$. Then the sequence $\left(x^{(n)}\right)$ is norm convergent and its limit lies in $\bigcap_{i \in I} C_{i}$, where $I:=\left\{i \in\{1, \ldots, N\}: \sum_{n} \mu_{i}^{(n)}=+\infty\right\}$.

REMARKS 4.20.

- Under the assumption on the relaxation parameters in the preceding examples, the condition $\underline{\lim }_{n: n \text { active for } i} \mu_{i}^{(n)}>0$ is equivalent to $\underline{\lim }_{n: n \text { active for } i} \lambda_{i}^{(n)}>0$ (cf. to Remark 3.13) and the condition $\sum_{n} \mu_{i}^{(n)}=+\infty$ is equivalent to $\sum_{n} \lambda_{i}^{(n)}=+\infty$ (cf. to Remark 3.26) for every index $i$.

- Example 4.18.(i) follows not only from Corollary 4.17 but also from Theorem 3.10 .

The next example shows that if one drops the assumption $\sum_{n} \mu_{i}^{(n)}=+\infty$ in Example 4.19, then one cannot expect the limit of $\left(x^{(n)}\right)$ to lie in $C_{i}$.

EXAMPLE 4.21. Let $\left.\left.X:=\mathbb{R}, N:=2, C_{1}:=C_{1}^{(n)}: \equiv\right]-\infty, 0\right]$, and $C_{2}:=C_{2}^{(n)}: \equiv$ $\left[0,+\infty\left[\right.\right.$. Suppose $x^{(0)}>0, \alpha_{1}^{(n)}: \equiv \alpha_{2}^{(n)}: \equiv 3 / 2$, and $\lambda_{1}^{(n)}<2 / 3$ for all $n$. Then

$$
x^{(n)}=\left(1-\frac{3}{2} \lambda_{1}^{(n-1)}\right) \cdots\left(1-\frac{3}{2} \lambda_{1}^{(0)}\right) x^{(0)},
$$

and therefore

$$
\lim _{n} x^{(n)} \in C_{1} \Longleftrightarrow \lim _{n} x^{(n)}=0 \Longleftrightarrow \sum_{n} \mu_{1}^{(n)}=+\infty .
$$

THEOREM 4.22. Given a projection algorithm, suppose $\left(P_{i}^{(n)}\right)$ converges actively pointwise to $P_{i}$ for every index $i$. Suppose further there is some subsequence $\left(n^{\prime}\right)$ of $(n)$ s.t. for every index $i$,

$$
\alpha_{i}^{\left(n^{\prime}\right)} \longrightarrow \alpha_{i} \text { and } \lambda_{i}^{\left(n^{\prime}\right)} \longrightarrow \lambda_{i}
$$

for some $\left.\left.\alpha_{i} \in\right] 0,2\right]$ and $\left.\left.\lambda_{i} \in\right] 0,1\right]$. If the interior of $C$ is nonempty, then the sequence $\left(x^{(n)}\right)$ converges in norm to some point in $C$. 
Proof. By Corollary 3.3.(i), $\left(x^{(n)}\right)$ converges in norm to some point $x$. We must show that $x \in C$.

Claim:

$$
P_{i}^{\left(n^{\prime}\right)} x^{\left(n^{\prime}\right)} \longrightarrow P_{i} x \quad \text { for every index } i
$$

Because $\left\|P_{i}^{\left(n^{\prime}\right)} x^{\left(n^{\prime}\right)}-P_{i}^{\left(n^{\prime}\right)} x\right\| \leq\left\|x^{\left(n^{\prime}\right)}-x\right\|$, we have $P_{i}^{\left(n^{\prime}\right)} x^{\left(n^{\prime}\right)}-P_{i}^{\left(n^{\prime}\right)} x \longrightarrow 0$. Since $\lambda_{i}^{\left(n^{\prime}\right)} \longrightarrow \lambda_{i}>0$, we see that $i$ is active at $n^{\prime}$ for all large $n^{\prime}$. The assumption on $\left(P_{i}^{(n)}\right)$ implies $P_{i}^{\left(n^{\prime}\right)} x \longrightarrow P_{i} x$. The claim follows.

Now

$$
x^{\left(n^{\prime}+1\right)}=\sum_{i=1}^{N} \lambda_{i}^{\left(n^{\prime}\right)}\left(\left(1-\alpha_{i}^{\left(n^{\prime}\right)}\right) x^{\left(n^{\prime}\right)}+\alpha_{i}^{\left(n^{\prime}\right)} P_{i}^{\left(n^{\prime}\right)} x^{\left(n^{\prime}\right)}\right)
$$

hence, by taking limits along the subsequence $\left(x^{\left(n^{\prime}\right)}\right)$ and by the claim,

$$
x=\sum_{i=1}^{N} \lambda_{i}\left(\left(1-\alpha_{i}\right) x+\alpha_{i} P_{i} x\right)
$$

or

$$
x=\sum_{i=1}^{N}\left(\frac{\lambda_{i} \alpha_{i}}{\sum_{j=1}^{N} \lambda_{j} \alpha_{j}}\right) P_{i} x .
$$

Proposition 2.12 implies that $x \in C$; the proof is complete.

EXAMPLE 4.23 (Butnariu and Censor's framework [20, Thm. 4.4]). Suppose $X$ is finite dimensional, the projection algorithm has constant sets, and the relaxation parameters depend only on $n$, say $\alpha_{i}^{(n)} \equiv \alpha^{(n)}$ for every index $i$ and all $n$. Suppose further there is some subsequence $\left(n^{\prime}\right)$ of $(n)$ s.t. for every index $i, \lambda_{i}^{\left(n^{\prime}\right)} \longrightarrow \lambda_{i}$ for some $\lambda_{i}>0$.

(i) If there is some $\epsilon>0$ s.t. $\epsilon \leq \alpha^{(n)} \leq 2-\epsilon$ for all large $n$, then the sequence $\left(x^{(n)}\right)$ converges in norm to some point in $C$.

(ii) If the interior of $C$ is nonempty and there is some subsequence $\left(n^{\prime \prime}\right)$ of $\left(n^{\prime}\right)$ s.t. $\alpha^{\left(n^{\prime \prime}\right)} \longrightarrow 2$, then the sequence $\left(x^{(n)}\right)$ converges in norm to some point in $C$.

Proof. (i): The assumption on the weights implies $\sum_{n} \mu_{i}^{(n)}=+\infty$ for every index $i$. Thus (i) follows from Example 4.19. (ii) is immediate from Theorem 4.22.

REMARK 4.24. Note that the last theorem works especially when $\alpha_{i}^{(n)} \equiv 2$. Since in this case $\mu_{i}^{(n)} \equiv 0$, none of the previous results are applicable. If we drop the assumption that int $C \neq \emptyset$, then the conclusion of the last theorem need not hold; see Example 3.6.

DEFINITION 4.25 (control). We say the projection algorithm considers remotest sets if for every $n$, at least one remotest index is active; i.e.,

$$
I_{\text {rem }}^{(n)}:=\left\{i: d\left(x^{(n)}, C_{i}\right)=\max \left\{d\left(x^{(n)}, C_{j}\right): j=1 \ldots N\right\}\right\} \cap I^{(n)} \neq \emptyset .
$$

Following Censor [21], we speak of remotest set control if the projection algorithm is singular and considers remotest sets. Obviously,

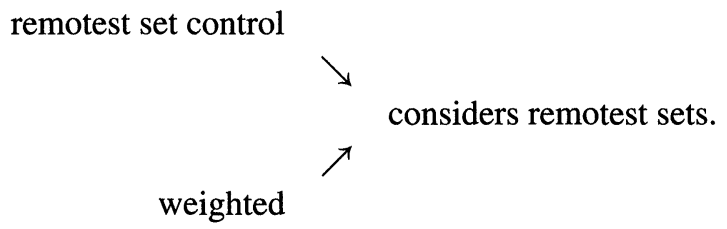

THEOREM 4.26 (weak topology results). Suppose the projection algorithm is strongly focusing and considers remotest sets. Suppose further that $\left(i^{(n)}\right)$ is a sequence of active remotest indices; i.e., $i^{(n)} \in I_{\text {rem }}^{(n)}$ for all $n$. 
(i) If $\sum_{n} \mu_{i^{(n)}}^{(n)}=+\infty$, then there is a subsequence $\left(x^{\left(n_{k}\right)}\right)_{k}$ of $\left(x^{(n)}\right)$ s.t.

$$
\max \left\{d\left(x^{\left(n_{k}\right)}, C_{j}\right): j=1 \ldots N\right\} \longrightarrow 0,
$$

and $\left(x^{\left(n_{k}\right)}\right)_{k}$ converges weakly to the unique weak cluster point of $\left(x^{(n)}\right)$ in $C$.

(ii) If $\varliminf_{n} \mu_{i^{(n)}}^{(n)}>0$, then $\left(x^{(n)}\right)$ converges weakly to some point in $C$ and

$$
\max \left\{d\left(x^{(n)}, C_{j}\right): j=1 \ldots N\right\} \longrightarrow 0 .
$$

Proof. (i): By Lemma 3.2.(iv), the series $\sum_{n} \mu_{i^{(n)}}^{(n)} d^{2}\left(x^{(n)}, C_{i^{(n)}}^{(n)}\right)$ is convergent. Hence $\underline{\lim }_{n} d\left(x^{(n)}, C_{i^{(n)}}^{(n)}\right)=0$. Thus we can extract a subsequence $\left(x^{\left(n_{k}\right)}\right)_{k}$ and fix an index $i$ s.t. $d\left(x^{\left(n_{k}\right)}, C_{i}^{\left(n_{k}\right)}\right) \longrightarrow 0, i^{\left(n_{k}\right)} \equiv i$, and $\left(x^{\left(n_{k}\right)}\right)$ converges weakly. Since the algorithm is strongly focusing and considers remotest sets, we conclude that

$$
\max \left\{d\left(x^{\left(n_{k}\right)}, C_{j}\right): j=1 \ldots N\right\} \longrightarrow 0 .
$$

By weak lower semicontinuity of $d\left(\cdot, C_{j}\right)$ for every index $j$, the weak limit of $\left(x^{\left(n_{k}\right)}\right)$ lies in $C$. By Theorem 2.16.(ii), $\left(x^{(n)}\right)$ has at most one weak cluster point in $C$; therefore, (i) is verified. (ii) is proved similarly.

REMARK 4.27. Remotest set control is an old and successful concept. In 1954, Agmon [1] and Motzkin and Schoenberg [83] studied projection algorithms for solving linear inequalities using remotest set control. Bregman [16] considered the situation when there is an arbitrary collection of intersecting closed convex sets. We will recapture Agmon's main result [1, Thm. 3] and some generalizations in $\S 6$.

5. Guaranteeing norm or linear convergence: Regularities. We uphold the notation of the preceding sections; in particular, we remember that $C_{1}, \ldots, C_{N}$ are closed convex sets with nonempty intersection $C$.

\section{Norm convergence and (bounded) regularity.}

DEFINITION 5.1. We say that the $N$-tuple of closed convex sets $\left(C_{1}, \ldots, C_{N}\right)$ is regular if

$$
\begin{aligned}
& \underset{\epsilon>0}{\forall} \underset{\delta>0}{\exists} \quad \underset{x \in X:}{\forall} \quad d(x, C) \leq \epsilon . \\
& \max \left\{d\left(x, C_{j}\right): j=1 \ldots N\right\} \leq \delta
\end{aligned}
$$

If this holds only on bounded sets, i.e.,

$$
\begin{aligned}
& \underset{X \supseteq S \text { bounded }}{\forall} \underset{\epsilon>0}{\forall} \underset{\delta}{\exists} \quad \underset{x \in S:}{\forall} \quad d(x, C) \leq \epsilon, \\
& \max \left\{d\left(x, C_{j}\right): j=1 \ldots N\right\} \leq \delta
\end{aligned}
$$

then we speak of a boundedly regular $N$-tuple $\left(C_{1}, \ldots, C_{N}\right)$.

Although the definition of (bounded) regularity is independent of the order of the sets, we prefer to think of $C_{1}, \ldots, C_{N}$ as a tuple. The geometric idea behind this definition is extremely simple: "If you are close to all sets, then the intersection cannot be too far away." In [13], we utilized this notion to formulate some norm convergence results for von Neumann's alternating projection algorithm for two sets.

The results of this subsection will illustrate the usefulness of this concept in our present framework. 
THEOREM 5.2. Suppose the projection algorithm is strongly focusing and p-intermittent for some positive integer $p$. Suppose further the $N$-tuple $\left(C_{1}, \ldots, C_{N}\right)$ is boundedly regular. Let

$$
v_{n}:=\min \left\{\mu_{i}^{(l)}: n p \leq l \leq(n+1) p-1 \text { and } i \text { active at } l\right\} \text { for all } n \geq 0 .
$$

If $\sum_{n} v_{n}=+\infty$, then the sequence $\left(x^{(n)}\right)$ converges in norm to some point in $C$. In particular, this happens whenever $\underline{\lim }_{n: n}$ active for $i \mu_{i}^{(n)}>0$ for every index $i$.

Proof. By Theorem 3.20.(ii), we obtain a subsequence $\left(x^{\left(n_{k} p\right)}\right)_{k}$ of $\left(x^{(n)}\right)$ s.t. $\left(x^{\left(n_{k} p\right)}\right)_{k}$ converges weakly to the unique weak cluster point of $\left(x^{(n)}\right)$ in $C$, say $x$,

$$
\begin{gathered}
\sum_{l=n_{k} p}^{\left(n_{k}+1\right) p-1} \sum_{i \in I^{(l)}} d\left(x^{(l)}, C_{i}^{(l)}\right) \longrightarrow 0, \quad \text { and } \\
x^{\left(n_{k} p+r_{k}\right)}-x^{\left(n_{k} p\right)} \longrightarrow 0
\end{gathered}
$$

for all sequences $\left(r_{k}\right)$ in $\{0, \ldots, p-1\}$. Fix any index $i$. Because the projection algorithm is intermittent, we get a sequence $\left(r_{k}\right)$ in $\{0, \ldots, p-1\}$ s.t. $i \in I^{\left(n_{k} p+r_{k}\right)}$ for all $k$. Then, by $(*), d\left(x^{\left(n_{k} p+r_{k}\right)}, C_{i}^{\left(n_{k} p+r_{k}\right)}\right) \longrightarrow 0$. Since $\left(x^{\left(n_{k} p+r_{k}\right)}\right)_{k}$ also converges to $x$ (by $\left.(* *)\right)$ and the projection algorithm is strongly focusing, we deduce that

$$
d\left(x^{\left(n_{k} p+r_{k}\right)}, C_{i}\right) \longrightarrow 0 .
$$

Hence, by $(* *), d\left(x^{\left(n_{k} p\right)}, C_{i}\right) \longrightarrow 0$. Since $i$ has been chosen arbitrarily, we actually have

$$
\max \left\{d\left(x^{\left(n_{k} p\right)}, C_{j}\right): j=1 \ldots N\right\} \longrightarrow 0 .
$$

Now $\left(C_{1}, \ldots, C_{N}\right)$ is boundedly regular and $\left(x^{\left(n_{k} p\right)}\right)_{k}$ is bounded; consequently, $d\left(x^{\left(n_{k} p\right)}, C\right) \longrightarrow 0$. Therefore, by Corollary 3.3.(ii), $\left(x^{(n)}\right)$ converges in norm to $x$.

THEOREM 5.3. Suppose the projection algorithm is strongly focusing and considers remotest sets. Suppose further the $N$-tuple $\left(C_{1}, \ldots, C_{N}\right)$ is boundedly regular and $\left(i^{(n)}\right)$ is a sequence of active remotest indices. If $\sum_{n} \mu_{i^{(n)}}^{(n)}=+\infty$, then the sequence $\left(x^{(n)}\right)$ converges in norm to some point in $C$. In particular, this happens whenever $\underline{\lim }_{n} \mu_{i^{(n)}}^{(n)}>0$.

Proof. By Theorem 4.26.(i), there exists a weakly convergent subsequence $\left(x^{\left(n_{k}\right)}\right)_{k}$ of $\left(x^{(n)}\right)$ with $\max \left\{d\left(x^{\left(n_{k}\right)}, C_{j}\right): j=1 \ldots N\right\} \longrightarrow 0$. Since $\left(C_{1}, \ldots, C_{N}\right)$ is boundedly regular, we get $d\left(x^{\left(n_{k}\right)}, C\right) \longrightarrow 0$. Now apply Corollary 3.3.(ii).

In order to make use of these theorems, we must know when an $N$-tuple $\left(C_{1}, \ldots, C_{N}\right)$ is boundedly regular. Fortunately, our observations on bounded regularity of a pair in [13] generalize easily to the $N$-set case.

PROPOSITION 5.4. regular.

(i) If some set $C_{i}$ is boundedly compact, then the $N$-tuple $\left(C_{1}, \ldots, C_{N}\right)$ is boundedly

(ii) If the $N$-tuple $\left(C_{1}, \ldots, C_{N}\right)$ is boundedly regular and some set $C_{i}$ is bounded, then $\left(C_{1}, \ldots, C_{N}\right)$ is regular.

(iii) If $X$ is finite dimensional, then every $N$-tuple $\left(C_{1}, \ldots, C_{N}\right)$ is boundedly regular.

Proof. An easy modification of [13, Thm. 3.9 (resp., Thm. 3.15) given for two sets] yields (i) (resp., (ii)). (iii) follows from (i).

REMARK 5.5. We gave an example [13, Ex. 5.5] of a pair which is not boundedly regular; therefore, bounded regularity requires some assumption. 
Linear convergence and (bounded) linear regularity. The following stronger and more quantitative version of (bounded) regularity allows us to discuss rates of convergence.

DEFINITION 5.6. We say that the $N$-tuple of closed convex sets $\left(C_{1}, \ldots, C_{N}\right)$ is linearly regular if

$$
\underset{\kappa>0}{\exists} \underset{x \in X}{\forall} d(x, C) \leq \kappa \max \left\{d\left(x, C_{j}\right): j=1 \ldots N\right\} .
$$

Again, if this holds only on bounded sets, i.e.,

$$
\underset{X \supseteq S \text { bounded } \kappa_{S}>0}{\exists} \underset{x \in S}{\forall} d(x, C) \leq \kappa_{S} \max \left\{d\left(x, C_{j}\right): j=1 \ldots N\right\},
$$

then we say that $\left(C_{1}, \ldots, C_{N}\right)$ is boundedly linearly regular.

Clearly,

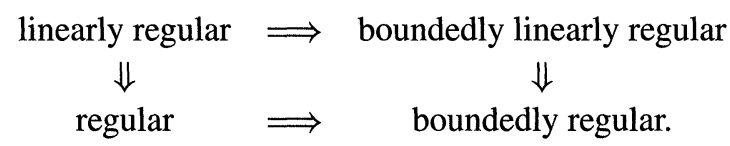

THEOREM 5.7. Suppose the projection algorithm is linearly focusing and intermittent. Suppose further the $N$-tuple $\left(C_{1}, \ldots, C_{N}\right)$ is boundedly linearly regular and there is some $\epsilon>0$ s.t. $\epsilon \leq \alpha_{i}^{(n)} \leq 2-\epsilon$ and $\epsilon \leq \lambda_{i}^{(n)}$ for all large $n$ and every index $i$ active at $n$. Then the sequence $\left(x^{(n)}\right)$ converges linearly to some point in $C$; the rate of convergence is independent of the starting point whenever $\left(C_{1}, \ldots, C_{N}\right)$ is linearly regular.

Proof. Suppose the projection algorithm is $p$-intermittent. Fix any index $i$. Then, for all $k \geq 0$, we get $m_{k}$ with $k p \leq m_{k} \leq(k+1) p-1$ and $i \in I^{\left(m_{k}\right)}$. Now $x^{\left(m_{k}\right)}=$ $A^{\left(m_{k}-1\right)} \cdots A^{(k p)} x^{(k p)}$ and, by Lemma 2.4.(iv) and Proposition 2.12.(ii),

$A^{(n)}$ is $\min \left\{\left(2-\alpha_{j}^{(n)}\right) / \alpha_{j}^{(n)}: j\right.$ active at $\left.n\right\}$-attracting w.r.t. $C$ for all $n \geq 0$.

Hence, by the assumption on the relaxation parameters, $A^{(n)}$ is $\epsilon / 2$-attracting w.r.t. $C$ for all large $n$. Thus, by Proposition 2.10.(ii),

$$
A^{\left(m_{k}-1\right)} \cdots A^{(k p)} \text { is } \frac{\epsilon}{2^{p-1}} \text {-attracting w.r.t. } C \quad \text { for all large } k .
$$

Since the projection algorithm is linearly focusing, there is some $\beta>0$ s.t. $\beta d\left(x^{(n)}, C_{j}\right) \leq$ $d\left(x^{(n)}, C_{j}^{(n)}\right)$ for all large $n$ and every index $j$ active at $n$. Now

$$
\begin{aligned}
d^{2}\left(x^{(k p)}, C_{i}\right) & \leq\left(\left\|x^{(k p)}-x^{\left(m_{k}\right)}\right\|+d\left(x^{\left(m_{k}\right)}, C_{i}\right)\right)^{2} \\
& \leq 2\left\|x^{(k p)}-x^{\left(m_{k}\right)}\right\|^{2}+2 d^{2}\left(x^{\left(m_{k}\right)}, C_{i}\right)
\end{aligned}
$$

here the first inequality follows from the nonexpansivity of $d\left(\cdot, C_{i}\right)$ and the second one is just " $(a+b)^{2} \leq 2 a^{2}+2 b^{2}$." Fix an arbitrary point $c \in C$. On the one hand, for all large $k$,

$$
\begin{aligned}
\left\|x^{(k p)}-x^{\left(m_{k}\right)}\right\|^{2} & =\left\|A^{\left(m_{k}-1\right)} \cdots A^{(k p)} x^{(k p)}-x^{(k p)}\right\|^{2} \\
& \leq \frac{2^{p-1}}{\epsilon}\left(\left\|x^{(k p)}-c\right\|^{2}-\left\|x^{\left(m_{k}\right)}-c\right\|^{2}\right) \\
& \leq \frac{2^{p-1}}{\epsilon}\left(\left\|x^{(k p)}-c\right\|^{2}-\left\|x^{((k+1) p)}-c\right\|^{2}\right) .
\end{aligned}
$$


On the other hand, by the assumptions on $\beta$, relaxation parameters, weights, and by Lemma 3.2.(ii) and Remark 3.13, we estimate for all large $k$ that

$$
\begin{aligned}
d^{2}\left(x^{\left(m_{k}\right)}, C_{i}\right) & \leq \frac{1}{\beta^{2}} d^{2}\left(x^{\left(m_{k}\right)}, C_{i}^{\left(m_{k}\right)}\right) \\
& =\frac{1}{\beta^{2}}\left\|x^{\left(m_{k}\right)}-P_{i}^{\left(m_{k}\right)} x^{\left(m_{k}\right)}\right\|^{2} \\
& \leq \frac{1}{\beta^{2} \mu_{i}^{\left(m_{k}\right)}}\left(\left\|x^{\left(m_{k}\right)}-c\right\|^{2}-\left\|x^{\left(m_{k}+1\right)}-c\right\|^{2}\right) \\
& \leq \frac{1}{\beta^{2} \epsilon^{3}}\left(\left\|x^{\left(m_{k}\right)}-c\right\|^{2}-\left\|x^{\left(m_{k}+1\right)}-c\right\|^{2}\right) \\
& \leq \frac{1}{\beta^{2} \epsilon^{3}}\left(\left\|x^{(k p)}-c\right\|^{2}-\left\|x^{((k+1) p)}-c\right\|^{2}\right)
\end{aligned}
$$

altogether

$$
d^{2}\left(x^{(k p)}, C_{i}\right) \leq\left(\frac{2^{p}}{\epsilon}+\frac{2}{\beta^{2} \epsilon^{3}}\right)\left(\left\|x^{(k p)}-c\right\|^{2}-\left\|x^{((k+1) p)}-c\right\|^{2}\right)
$$

which, after choosing $c:=P_{C} x^{(k p)}$, yields

$$
d^{2}\left(x^{(k p)}, C_{i}\right) \leq\left(\frac{2^{p}}{\epsilon}+\frac{2}{\beta^{2} \epsilon^{3}}\right)\left(d^{2}\left(x^{(k p)}, C\right)-d^{2}\left(x^{((k+1) p)}, C\right)\right) .
$$

Because $i$ has been chosen arbitrarily, the last estimate is true for every index $i$, provided that $k$ is large enough. Since $\left(C_{1}, \ldots, C_{N}\right)$ is boundedly linearly regular, we obtain for $S:=\left\{x^{(n)}: n \geq 0\right\}$ a constant $\kappa_{S}>0$ s.t.

$$
d\left(x^{(n)}, C\right) \leq \kappa_{S} \max \left\{d\left(x^{(n)}, C_{j}\right): j=1 \ldots N\right\} \quad \text { for all } n \geq 0 .
$$

Note that if $\left(C_{1}, \ldots, C_{N}\right)$ is linearly regular, then the constant $\kappa_{S}$ can be chosen independent of $S$. Combining gives

$$
d^{2}\left(x^{(k p)}, C\right) \leq \kappa_{S}^{2}\left(\frac{2^{p}}{\epsilon}+\frac{2}{\beta^{2} \epsilon^{3}}\right)\left(d^{2}\left(x^{(k p)}, C\right)-d^{2}\left(x^{((k+1) p)}, C\right)\right) .
$$

Therefore, by Theorem 2.16.(vi) applied to $\left(x^{(k p)}\right)$, the sequence $\left(x^{(k p)}\right)$ converges linearly to some point $x$ in $C$. By Theorem 2.16.(i) and Proposition 1.6, the entire sequence $\left(x^{(n)}\right)$ converges linearly to $x$; the rate of convergence is independent of the starting point whenever $\left(C_{1}, \ldots, C_{N}\right)$ is linearly regular.

THEOREM 5.8. Suppose the projection algorithm is linearly focusing and considers remotest sets. Suppose further the $N$-tuple $\left(C_{1}, \ldots, C_{N}\right)$ is boundedly linearly regular and $\left(i^{(n)}\right)$ is a sequence of active remotest indices. If $\underline{\lim }_{n} \mu_{i^{(n)}}^{(n)}>0$, then the sequence $\left(x^{(n)}\right)$ converges linearly to some point in $C$; the rate of convergence is independent of the starting point whenever $\left(C_{1}, \ldots, C_{N}\right)$ is linearly regular.

Proof. First, since the projection algorithm is linearly focusing, we get $\beta>0$ s.t. $\beta d\left(x^{(n)}, C_{i}\right) \leq d\left(x^{(n)}, C_{i}^{(n)}\right)$ for all $n$ and every index $i$ active at $n$. Second, since $\left(C_{1}, \ldots, C_{N}\right)$ is boundedly linearly regular, we obtain for $S:=\left\{x^{(n)}: n \geq 0\right\}$ a constant $\kappa_{S}>0$ s.t. $d\left(x^{(n)}, C\right) \leq \kappa_{S} \max \left\{d\left(x^{(n)}, C_{j}\right): j=1 \ldots N\right\}$ for all $n \geq 0$. Once more, the constant $\kappa_{S}$ can be chosen independent of $S$ whenever $\left(C_{1}, \ldots, C_{N}\right)$ is linearly regular. Third, there is some 
$\epsilon>0$ s.t. $\mu_{i^{(n)}}^{(n)} \geq \epsilon$ for all large $n$. Putting this together and using Lemma 3.2.(ii), we estimate for all large $n$ that

$$
\begin{aligned}
d^{2}\left(x^{(n)}, C\right) & \leq \kappa_{S}^{2} \max \left\{d^{2}\left(x^{(n)}, C_{j}\right): j=1 \ldots N\right\} \\
& =\kappa_{S}^{2} d^{2}\left(x^{(n)}, C_{i^{(n)}}\right) \\
& \leq\left(\frac{\kappa_{S}}{\beta}\right)^{2}\left\|x^{(n)}-P_{i^{(n)}}^{(n)} x^{(n)}\right\|^{2} \\
& \leq\left(\frac{\kappa_{S}}{\beta}\right)^{2} \frac{1}{\mu_{i^{(n)}}^{(n)}}\left(\left\|x^{(n)}-P_{C} x^{(n)}\right\|^{2}-\left\|x^{(n+1)}-P_{C} x^{(n)}\right\|^{2}\right) \\
& \leq\left(\frac{\kappa_{S}}{\beta}\right)^{2} \frac{1}{\epsilon}\left(d^{2}\left(x^{(n)}, C\right)-d^{2}\left(x^{(n+1)}, C\right)\right) .
\end{aligned}
$$

Therefore, by Theorem 2.16.(vi), $\left(x^{(n)}\right)$ converges linearly to some point in $C$ (again with a rate independent of the starting point whenever $\left(C_{1}, \ldots, C_{N}\right)$ is linearly regular).

(Bounded) linear regularity: Examples. Having seen the power of (bounded) linear regularity, we now investigate this concept itself and provide basic prototypes.

PROPOSITION 5.9. If each set $C_{i}$ is a closed convex cone, then the following conditions are equivalent.

(i) $\left(C_{1}, \ldots, C_{N}\right)$ is regular.

(ii) $\left(C_{1}, \ldots, C_{N}\right)$ is linearly regular.

(iii) $\left(C_{1}, \ldots, C_{N}\right)$ is boundedly linearly regular.

Proof. Adapt the proof of [13, Thm. 3.17].

REMARK 5.10. It follows that (i), (ii), and (iii) are equivalent if

- $C_{1}, \ldots, C_{N}$ are closed convex translated cones with a common vertex (a simple translation argument),

- $C_{1}, \ldots, C_{N}$ are closed affine subspaces with nonempty intersection.

THEOREM 5.11 ((bounded) linear regularity: reduction to pairs). If each of the $N-1$ pairs

$$
\begin{aligned}
& \left(C_{1}, C_{2}\right) \\
& \left(C_{1} \cap C_{2}, C_{3}\right) \\
& \vdots \\
& \left(C_{1} \cap C_{2} \cap \cdots \cap C_{N-2}, C_{N-1}\right) \\
& \left(C_{1} \cap C_{2} \cap \cdots \cap C_{N-2} \cap C_{N-1}, C_{N}\right)
\end{aligned}
$$

is (boundedly) linearly regular, then so is the $N$-tuple $\left(C_{1}, \ldots, C_{N}\right)$.

Proof. We consider the case when all pairs are boundedly linearly regular; the case when all pairs are linearly regular is treated analogously. Fix a bounded set $S$ and get (by hypothesis) $\kappa_{1}, \ldots, \kappa_{N-1}>0$ s.t. for every $x \in S$, we have the estimates

$$
\begin{aligned}
d\left(x, C_{1} \cap C_{2}\right) & \leq \kappa_{1} \max \left\{d\left(x, C_{1}\right), d\left(x, C_{2}\right)\right\} \\
d\left(x, C_{1} \cap C_{2} \cap C_{3}\right) & \leq \kappa_{2} \max \left\{d\left(x, C_{1} \cap C_{2}\right), d\left(x, C_{3}\right)\right\}, \\
& \vdots \\
d\left(x, C_{1} \cap \cdots \cap C_{N}\right) & \leq \kappa_{N-1} \max \left\{d\left(x, C_{1} \cap \cdots \cap C_{N-1}\right), d\left(x, C_{N}\right)\right\}
\end{aligned}
$$

hence $d\left(x, C_{1} \cap \cdots \cap C_{N}\right) \leq \kappa_{1} \kappa_{2} \cdots \kappa_{N-1} \max \left\{d\left(x, C_{j}\right): j=1 \ldots N\right\}$. 
For bounded linear regularity of pairs, we gave the following sufficient condition (see [13, Cor. 4.5]).

FACT 5.12. Suppose $E, F$ are two closed convex sets. If $0 \in$ icr $(E-F)$, then the pair $(E, F)$ is boundedly linearly regular. In particular, this happens whenever

(i) $0 \in$ int $(E-F)$ or

(ii) $E-F$ is a closed subspace.

Combining the two preceding results immediately yields the following.

COROLLARY 5.13. If

$0 \in \operatorname{icr}\left(C_{1}-C_{2}\right) \cap \operatorname{icr}\left(\left(C_{1} \cap C_{2}\right)-C_{3}\right) \cap \cdots \cap \operatorname{icr}\left(\left(C_{1} \cap \cdots \cap C_{N-1}\right)-C_{N}\right)$,

then the $N$-tuple $\left(C_{1}, \ldots, C_{N}\right)$ is boundedly linearly regular.

CoROLlary 5.14. If $C_{N} \cap$ int $\left(C_{1} \cap \cdots \cap C_{N-1}\right) \neq \emptyset$, then $\left(C_{1}, \ldots, C_{N}\right)$ is boundedly linearly regular.

REMARK 5.15. These sufficient conditions for bounded linear regularity do depend on the order of the sets, whereas bounded linear regularity does not. Consequently, these conditions might still be applicable after a suitable permutation of the sets.

In applications, the $N$ sets almost always have additional structure. One important case is when all sets are closed subspaces. In the following, we will completely characterize regularity of an $N$-tuple of closed subspaces. We begin with the case when $N=2$.

Recall that the angle $\gamma=\gamma\left(C_{1}, C_{2}\right) \in[0, \pi / 2]$ between two subspaces $C_{1}, C_{2}$ is given by (see Friedrichs [54] or Deutsch [42, 43])

$$
\cos \gamma=\sup \left\{\left\langle c_{1}, c_{2}\right\rangle: c_{1} \in C_{1} \cap\left(C_{1} \cap C_{2}\right)^{\perp}, c_{2} \in C_{2} \cap\left(C_{1} \cap C_{2}\right)^{\perp},\left\|c_{1}\right\|=\left\|c_{2}\right\|=1\right\} .
$$

PROPOSITION 5.16. If $C_{1}, C_{2}$ are two closed subspaces and $\gamma$ is the angle between them, then the following conditions are equivalent.

(i) $\gamma>0$.

(ii) $C_{1}+C_{2}$ is closed.

(iii) $C_{1}^{\perp}+C_{2}^{\perp}$ is closed.

(iv) $\left(C_{1}, C_{2}\right)$ is linearly regular.

(v) $\left(C_{1}, C_{2}\right)$ is boundedly linearly regular.

(vi) $\left(C_{1}, C_{2}\right)$ is regular.

(vii) $\left(C_{1}, C_{2}\right)$ is boundedly regular.

Proof. "(i) $\Longleftrightarrow$ (ii)" is due to Deutsch [42, Lem. 2.5.(4)] and Simonič ([93], a proof can be found in [13, Lem. 4.10]). "(ii) $\Longleftrightarrow$ (iii)" is well known (see Jameson's [70, Cor. 35.6]).

"(ii) $\Longrightarrow($ iv $) \Longleftrightarrow(v) \Longleftrightarrow(v i) \Longrightarrow$ (vii)": Combine Fact 5.12.(ii) and Proposition 5.9.

"(vii) $\Longrightarrow($ i)": Let us prove the contrapositive. Suppose $\gamma=0$. Then we obtain two sequences $\left(c_{1}^{(n)}\right),\left(c_{2}^{(n)}\right)$ with

$$
c_{1}^{(n)} \in C_{1}, \quad c_{2}^{(n)} \in C_{2}, \quad\left\|c_{1}^{(n)}\right\|=\left\|c_{2}^{(n)}\right\|=1, \quad P_{C_{1} \cap C_{2}} c_{1}^{(n)}=P_{C_{1} \cap C_{2}} c_{2}^{(n)}=0
$$

for every $n$, and

$$
\left\langle c_{1}^{(n)}, c_{2}^{(n)}\right\rangle \longrightarrow 1=\cos 0 .
$$

Expanding $\left\|c_{1}^{(n)}-c_{2}^{(n)}\right\|^{2}$ yields $c_{1}^{(n)}-c_{2}^{(n)} \longrightarrow 0$. On the one hand, if we define $x^{(n)}:=$ $\left(c_{1}^{(n)}+c_{2}^{(n)}\right) / 2$, then, by the parallelogram law,

$$
\left\|x^{(n)}\right\| \longrightarrow 1 \text {. }
$$

On the other hand,

$$
P_{C_{1} \cap C_{2}} x^{(n)} \equiv 0, x^{(n)}-c_{1}^{(n)} \longrightarrow 0, x^{(n)}-c_{2}^{(n)} \longrightarrow 0 .
$$


Altogether, $\left(x^{(n)}\right)$ is bounded, $d\left(x^{(n)}, C_{1}\right) \longrightarrow 0, d\left(x^{(n)}, C_{2}\right) \longrightarrow 0$, but $d\left(x^{(n)}, C_{1} \cap C_{2}\right)=$ $\left\|x^{(n)}\right\| \longrightarrow 1$. Therefore, $\left(C_{1}, C_{2}\right)$ is not boundedly regular and the proof is complete.

A short excursion into a useful product space. We build - in the spirit of Pierra [85]the product space

$$
\mathbf{X}:=\prod_{i=1}^{N}\left(X, \frac{1}{N}\langle\cdot, \cdot\rangle\right)
$$

and define the diagonal

$$
\Delta:=\left\{\left(x_{1}, \ldots, x_{N}\right) \in \mathbf{X}: x_{1}=x_{2}=\cdots=x_{N} \in X\right\}
$$

and the product

$$
\mathbf{B}:=\prod_{i=1}^{N} C_{i}
$$

This allows us to identify the set $C$ with $\Delta \cap \mathbf{B}$. Then (see, for example, [85]) for $\mathbf{x}, \mathbf{y} \in \mathbf{X}$, we have

$$
\|\mathbf{x}-\mathbf{y}\|^{2}=\sum_{i=1}^{N} \frac{1}{N}\left\|x_{i}-y_{i}\right\|^{2}
$$

and the projections onto $\Delta$ and $B$ are given by

$$
\begin{aligned}
P_{\Delta}\left(x_{1}, x_{2}, \ldots, x_{N}\right) & =\left(\sum_{i=1}^{N} \frac{1}{N} x_{i}, \sum_{i=1}^{N} \frac{1}{N} x_{i}, \ldots, \sum_{i=1}^{N} \frac{1}{N} x_{i}\right), \\
P_{\mathbf{B}}\left(x_{1}, x_{2}, \ldots, x_{N}\right) & =\left(P_{1} x_{1}, P_{2} x_{2}, \ldots, P_{N} x_{N}\right) .
\end{aligned}
$$

PROPOSITION 5.17. If $\mathbf{0} \in \mathrm{icr}(\Delta-\mathbf{B})$, then $(\Delta, \mathbf{B})$ is boundedly linearly regular.

Proof. This is nothing but Fact 5.12 applied in $\mathbf{X}$.

We now tackle the $N$-subspace case.

LEMMA 5.18. If each set $C_{i}$ is a closed subspace, then the following conditions are equivalent.

(i) $C_{1}^{\perp}+\cdots+C_{N}^{\perp}$ is closed.

(ii) $\Delta+\mathbf{B}$ is closed.

(iii) $(\Delta, \mathbf{B})$ is (boundedly) (linearly) regular.

Proof. Denote $C_{1}^{\perp}+\cdots+C_{N}^{\perp}$ by $S$ and consider

$$
T: \mathbf{X} \longrightarrow X:\left(x_{1}, \ldots, x_{N}\right) \longmapsto x_{1}+\cdots+x_{N}
$$

Clearly, $T$ is onto and kernel $T=\Delta^{\perp}$. By a useful consequence of the open mapping theorem (see, for example, Holmes's [67, Lem. 17.H]),

$$
S \text { is closed } \Longleftrightarrow \Delta^{\perp}+\prod_{i=1}^{N} C_{i}^{\perp} \text { is closed. }
$$

Now apply Proposition 5.16 to $\Delta$ and $\mathbf{B}$ in $\mathbf{X}$.

THEOREM 5.19 (linear regularity and subspaces). If each set $C_{i}$ is a closed subspace, then the following conditions are equivalent.

(i) $C_{1}^{\perp}+\cdots+C_{N}^{\perp}$ is closed.

(ii) $\left(C_{1}, \ldots, C_{N}\right)$ is linearly regular.

(iii) $\left(C_{1}, \ldots, C_{N}\right)$ is boundedly linearly regular.

(iv) $\left(C_{1}, \ldots, C_{N}\right)$ is regular.

(v) $\left(C_{1}, \ldots, C_{N}\right)$ is boundedly regular. 
Proof. “(i) $\Longrightarrow($ ii)": By the last lemma, there is some $\kappa>0$ s.t.

$$
d(\mathbf{x}, \Delta \cap \mathbf{B}) \leq \kappa \max \{d(\mathbf{x}, \Delta), d(\mathbf{x}, \mathbf{B})\} \quad \text { for every } \mathbf{x} \in \mathbf{X} .
$$

In particular, if $x \in X$ and $\mathbf{x}:=(x, \ldots, x) \in \Delta$, then

$$
d^{2}(x, C)=d^{2}(\mathbf{x}, \Delta \cap \mathbf{B}) \leq \kappa^{2} d^{2}(\mathbf{x}, \mathbf{B}) ;
$$

therefore, the linear regularity of $\left(C_{1}, \ldots, C_{N}\right)$ follows from

$$
\begin{aligned}
d^{2}(x, C) & \leq \kappa^{2}\left(d^{2}\left(x, C_{1}\right)+\cdots+d^{2}\left(x, C_{N}\right)\right) / N \\
& \leq \kappa^{2} \max \left\{d^{2}\left(x, C_{i}\right): i=1 \ldots N\right\} .
\end{aligned}
$$

"(ii) $\Longleftrightarrow$ (iii) $\Longleftrightarrow$ (iv) $\Longrightarrow(v) "$ follows from Proposition 5.9.

"(v) $\Longrightarrow(\mathrm{i})$ ": We prove the contrapositive. Suppose $C_{1}^{\perp}+\cdots+C_{N}^{\perp}$ is not closed. Then, by the preceding lemma, $(\Delta, \mathbf{B})$ is not boundedly regular. We thus obtain a bounded sequence $\left(\mathbf{x}^{(n)}\right)$ s.t.

$$
d\left(\mathbf{x}^{(n)}, \Delta\right) \longrightarrow 0, d\left(\mathbf{x}^{(n)}, \mathbf{B}\right) \longrightarrow 0, \text { but } \underline{\lim }_{n} d\left(\mathbf{x}^{(n)}, \Delta \cap \mathbf{B}\right)>0 .
$$

Define $x^{(n)}$ to be the first coordinate of $P_{\Delta} \mathbf{x}^{(n)}$. Then the sequence $\left(x^{(n)}\right)$ is bounded, $\frac{1}{N} \sum_{i=1}^{N} d^{2}\left(x^{(n)}, C_{i}\right) \longrightarrow 0$, but $\varliminf_{n} d^{2}\left(x^{(n)}, \bigcap_{i=1}^{N} C_{i}\right)>0$. Therefore, $\left(C_{1}, \ldots, C_{N}\right)$ is not boundedly regular and the proof is complete.

REMARKS 5.20.

- Browder implicitly proved “(i) $\Longrightarrow$ (ii)" of the last theorem in $[17, \S 2]$.

- It is interesting that, unless $N=2$, the closedness of the sum $C_{1}+\cdots+C_{N}$ is not related to the regularity of the $N$-tuple $\left(C_{1}, \ldots, C_{N}\right)$. Indeed, for $N \geq 3$, take two closed subspaces $C_{1}, C_{2}$ with nonclosed sum. (i): Set $C_{3}:=\cdots:=C_{N}:=X$; then $\left(C_{1}, \ldots, C_{N}\right)$ is not regular (Proposition 5.16), but the sum $C_{1}+\cdots+C_{N}$ is closed. (ii): Set $C_{3}:=\ldots:=C_{N}:=\{0\}$; then $\left(C_{1}, \ldots, C_{N}\right)$ is regular, but the sum $C_{1}+\cdots+C_{N}$ is not closed. Altogether, the closedness of the sum $C_{1}+\cdots+C_{N}$ is neither necessary nor sufficient for regularity of the $N$-tuple $\left(C_{1}, \ldots, C_{N}\right)$.

- For closed intersecting affine subspaces, a corresponding version of the last theorem can be formulated (since regularity is preserved under translation of the sets by some fixed vector).

- Applying the last theorem to orthogonal complements yields the following characterization.

If each set $C_{i}$ is a closed subspace, then the following conditions are equivalent.

(i) $C_{1}+\cdots+C_{N}$ is closed.

(ii) There is some $\kappa>0$ s.t. for every $x \in X$,

$$
\left\|P_{\overline{C_{1}+\cdots+C_{N}}} x\right\| \leq \kappa\left(\left\|P_{C_{1}} x\right\|+\cdots+\left\|P_{C_{N}} x\right\|\right) .
$$

(iii) For every bounded sequence $\left(x^{(n)}\right)$,

$$
\max \left\{\left\|P_{C_{i}} x^{(n)}\right\|: i=1 \ldots N\right\} \longrightarrow 0 \text { implies }\left\|P_{\overline{C_{1}+\cdots+C_{N}}} x^{(n)}\right\| \longrightarrow 0 .
$$

COROLlaRY 5.21. Suppose each set $C_{i}$ is a closed subspace. Then the N-tuple $\left(C_{1}, \ldots, C_{N}\right)$ is linearly regular whenever

(i) at least one subspace is finite dimensional or

(ii) all subspaces except possibly one have finite co-dimension. 
Proof. (i): Using Proposition 5.16, induction on $N$, and the well-known fact that the sum of a closed subspace and a finite-dimensional subspace is closed (see, for example, Jameson's [70, Prop. 20.1]), we obtain readily that $C_{1}^{\perp}+\cdots+C_{N}^{\perp}$ is finite co-dimensional and closed. Now apply the last theorem.

(ii): If without loss of generality $C_{1}, \ldots, C_{N-1}$ are finite co-dimensional, then $C_{1}^{\perp}+\cdots+$ $C_{N-1}^{\perp}$ is finite dimensional. Again, $C_{1}^{\perp}+\cdots+C_{N}^{\perp}$ is closed and the last theorem applies.

Once more, an analogous version of the last corollary holds for closed intersecting affine subspaces. We state the most important case.

COROLLARY 5.22 (linear regularity and intersecting hyperplanes). If each set $C_{i}$ is $a$ hyperplane, then the $N$-tuple $\left(C_{1}, \ldots, C_{N}\right)$ is linearly regular.

We now give another important class of linearly regular $N$-tuples.

FACT 5.23 (linear regularity and intersecting halfspaces). If each set $C_{i}$ is a halfspace, then the $N$-tuple $\left(C_{1}, \ldots, C_{N}\right)$ is linearly regular.

REMARK 5.24. In 1952, Hoffman [66] proved this fact, relying on some results by Agmon [1] for Euclidean spaces. It turns out that his proof also works for Hilbert spaces; a detailed proof will appear in the thesis of the first author.

The following result shows how one builds more examples of (boundedly) (linearly) regular tuples.

PROPOSITION 5.25. Suppose $\left(C_{1}, \ldots, C_{N}\right)$ is a (boundedly) (linearly) regular $N$-tuple and $J_{1} \cup \ldots \cup J_{M}=\{1, \ldots, N\}$ is a disjoint decomposition of $\{1, \ldots, N\}$; i.e., $J_{m} \neq \emptyset$ and $J_{m} \cap J_{m^{\prime}}=\emptyset$ for $m, m^{\prime} \in\{1, \ldots, M\}$ and $m \neq m^{\prime}$. If we set

$$
D_{m}:=\bigcap_{i \in J_{m}} C_{i} \quad \text { for every } m \in\{1, \ldots, M\},
$$

then the $M$-tuple $\left(D_{1}, \ldots, D_{M}\right)$ is (boundedly) (linearly) regular.

Proof. Suppose $\left(C_{1}, \ldots, C_{N}\right)$ is linearly regular. Then there is some $\kappa>0$ s.t. $d\left(x, C_{1} \cap\right.$ $\left.\cdots \cap C_{N}\right) \leq \kappa \max _{n} d\left(x, C_{n}\right)$ for every $x \in X$; thus

$$
\begin{aligned}
d\left(x, D_{1} \cap \cdots \cap D_{M}\right) & =d\left(x, C_{1} \cap \cdots \cap C_{N}\right) \\
& \leq \kappa \max _{n} d\left(x, C_{n}\right) \\
& =\kappa \max _{m} \max _{n \in J_{m}} d\left(x, C_{n}\right) \\
& \leq \kappa \max _{m} d\left(x, D_{m}\right) .
\end{aligned}
$$

Therefore, $\left(D_{1}, \ldots, D_{M}\right)$ is linearly regular. The proofs of the remaining cases are similar and thus are omitted.

COROLLARY 5.26 (linear regularity and intersecting polyhedra). If each set $C_{i}$ is a polyhedron, then the $N$-tuple $\left(C_{1}, \ldots, C_{N}\right)$ is linearly regular.

We finish this section with a result on the "frequency" (in the sense of Baire category) of boundedly linear $N$-tuples. Quite surprisingly, "bounded linear regularity is the rule." Since we will not use this result in what follows, we only sketch a proof. For basic results on the Hausdorff metric, we recommend Klein and Thompson's [76, 44 ; for basic results on Baire category see, for example, Holmes's [67, §17].

THEOREM 5.27. Suppose $\mathcal{T}$ is the set of all $N$-tuples of the form $\left(C_{1}, \ldots, C_{N}\right)$, where each set $C_{i}$ is bounded closed convex and the intersection $\bigcap_{i=1}^{N} C_{i}$ is nonempty. Then the subset of all boundedly linearly regular $N$-tuples is residual in $\mathcal{T}$ (equipped with the Hausdorff metric).

Sketch of a Proof. We work in the product space $\mathbf{X}$.

Step 1: Show that $\mathcal{T}$ is a closed subset in the complete metric space consisting of all closed subsets of $\mathbf{X}$ equipped with the Hausdorff metric. 
Step 2: Denote by $\mathcal{R}$ the subset of $\mathcal{T}$ consisting of all boundedly linearly regular $N$-tuples. Deduce from Proposition 5.17 that if $\mathbf{B}=\left(C_{1}, \ldots, C_{N}\right) \in \mathcal{T}$ and $0 \in$ int $(\Delta-\mathbf{B})$, then $\mathbf{B} \in \mathcal{R}$.

Step 3: Define $\mathcal{O}:=\{\mathcal{B} \in \mathcal{T}: 0 \in \operatorname{int}(\Delta-\mathbf{B})\}$ and show that $\mathcal{O}$ is dense in $\mathcal{T}$ (given $\mathbf{B}=\left(C_{1}, \ldots, C_{N}\right) \in \mathcal{T}$, consider the "nearby" $\left(C_{1}+\epsilon B_{\mathbf{X}}, \ldots, C_{N}+\epsilon B_{\mathbf{X}}\right)$ in $\mathcal{O}$ for small $\epsilon>0)$.

Step 4: Prove that $\mathcal{O}$ is open in $\mathcal{T}$. Indeed, denote the Hausdorff metric by $h$, fix $\mathbf{B} \in \mathcal{O}$, and get $\epsilon>0$ s.t. $\epsilon B_{\mathbf{X}} \subseteq \Delta-\mathbf{B}$. Suppose $\mathbf{B}^{\prime} \in \mathcal{T}$ with $h\left(\mathbf{B}, \mathbf{B}^{\prime}\right)<\epsilon / 2$. Then

$$
\frac{\epsilon}{2} B_{\mathbf{X}}+\frac{\epsilon}{2} B_{\mathbf{X}}=\epsilon B_{\mathbf{X}} \subseteq \Delta-\mathbf{B} \subseteq \Delta-\mathbf{B}^{\prime}+\frac{\epsilon}{2} B_{\mathbf{X}} .
$$

By Rådström's cancellation lemma [87, Lem. 1],

$$
\frac{\epsilon}{2} B_{\mathbf{X}} \subseteq \Delta-\mathbf{B}^{\prime}
$$

thus $\mathbf{B}^{\prime} \in \mathcal{O}$.

Conclusion. $\mathcal{R}$ is residual in $\mathcal{T}$ because $\mathcal{R} \supseteq \mathcal{O}$ and $\mathcal{O}$ is open and dense in $\mathcal{T}$.

REMARK 5.28. In view of Theorems 5.7, 5.8, and 5.27, we can loosely say that "linear convergence is the rule for certain algorithms." The restriction that every $C_{i}$ be bounded is not really severe since a reduction to this case can be made as soon as the starting point is chosen.

6. Harvest time II: Examples. In this section, numerous examples for our results are given. To demonstrate the applicability of our framework, we mainly chose examples that are closely related to known results and only occasionally comment on (sometimes very substantial) possible generalizations.

"Fairly" general sets.

\section{Random control.}

EXAMPLE 6.1. Suppose the projection algorithm is linearly focusing and some set $C_{j}$ is boundedly compact. Suppose further that

(i) $\varliminf_{n: n \text { active for } i} \mu_{i}^{(n)}>0$ for every index $i$ or

(ii) there is some $\epsilon>0$ s.t. $\epsilon \leq \alpha_{i}^{(n)} \leq 2-\epsilon$ and $\sum_{n: n \text { active for } i} \mu_{i}^{(n)}=+\infty$ for every index $i$ and all large $n$ active for $i$.

Then the sequence $\left(x^{(n)}\right)$ converges in norm to some point in $C$.

Proof. By Lemma 3.2.(iv), the sum $\sum_{n: n \text { active for } j} \mu_{j}^{(n)} d^{2}\left(x^{(n)}, C_{j}^{(n)}\right)$ is finite. Since the projection algorithm is linearly focusing, the assumptions on $\left(\mu_{i}^{(n)}\right)$ imply the existence of a subsequence $\left(x^{\left(n^{\prime}\right)}\right)$ of $\left(x^{(n)}\right)$ with

$$
\left\|x^{\left(n^{\prime}\right)}-P_{j} x^{\left(n^{\prime}\right)}\right\|=d\left(x^{\left(n^{\prime}\right)}, C_{j}\right) \longrightarrow 0 \text { and } j \text { is active at } n^{\prime} .
$$

After passing to a subsequence if necessary, we can assume that $\left(P_{j} x^{\left(n^{\prime}\right)}\right)$ is norm convergent; hence, so is $\left(x^{\left(n^{\prime}\right)}\right)$. Therefore, the sequence $\left(x^{(n)}\right)$ has a norm cluster point. If (i) holds, then the result follows from Theorem 3.10. Otherwise (ii) holds and then the result follows from Theorems 4.15 and 3.20.(iii).

REMARKS 6.2.

- This result improves Examples 4.6, 4.18.(i), and 4.19.

- As we commented in Remarks 4.7, the problem becomes much harder without a compactness assumption. Nevertheless, some interesting results were obtained by Bruck [18], Youla [107], and Dye and Reich [48].

An immediate consequence of Example 6.1 is the following.

EXAMPLE 6.3 (Bruck's [18, Cor. 1.2]). Suppose the projection algorithm is singular and has constant sets where (at least) one is boundedly compact. If there is some $\epsilon>0$ s.t. 
$\epsilon \leq \alpha_{i}^{(n)} \equiv \alpha_{i} \leq 2-\epsilon$ for every index $i$, then the sequence $\left(x^{(n)}\right)$ converges in norm to some point in $C$.

REMARK 6.4. Bruck's proof is quite different and is highly recommended.

Intermittent control. We start with some results on linear convergence.

EXAMPLE 6.5 (Browder's [17, Thm. 3]). Suppose the projection algorithm is almost cyclic, unrelaxed, and has constant sets. Suppose further that $0 \in C$ and for every $r>0$, there is some $\kappa_{r}>0$ s.t.

$$
\|x\| \leq \kappa_{r} \max \left\{d\left(x, C_{i}\right): i=1 \ldots N\right\} \text { for all } x \in r B_{X} .
$$

Then $C=\{0\}$ and the sequence $\left(x^{(n)}\right)$ converges linearly to 0 .

Proof. By (*), obviously $C \cap r B_{X}=\{0\}$. Since $r$ can be chosen arbitrarily large, it follows that $C=\{0\}$. Thus $(*)$ states that $d(x, C)=\|x\| \leq \max \left\{d\left(x, C_{i}\right): i=1 \ldots N\right\}$; i.e., the $N$-tuple $\left(C_{1}, \ldots, C_{N}\right)$ is boundedly linearly regular. The result now follows from Theorem 5.7.

EXAMPLE 6.6 (Youla and Webb's [108, Thm. 3]). Suppose the projection algorithm is cyclic and has constant sets. Suppose further the relaxation parameters satisfy $0<\alpha_{i}^{(n)} \equiv$ $\alpha_{i}<2$ for every index $i$ and all $n$ active for $i$. If there is some index $j \in\{1, \ldots, N\}$ s.t.

$$
C_{j} \cap \operatorname{int} \bigcap_{i \in\{1, \ldots, N\} \backslash\{j\}} C_{i} \neq \emptyset,
$$

then the sequence $\left(x^{(n)}\right)$ converges linearly to some point in $C$.

Proof. By Corollary 5.14 (cf. Remark 5.15), the $N$-tuple $\left(C_{1}, \ldots, C_{N}\right)$ is boundedly linearly regular. Now apply Theorem 5.7.

REMARK 6.7. An extended version of Youla and Webb's well-written paper is Youla's [106].

Analogously, we can prove the following.

EXAMPLE 6.8 (Gubin, Polyak, and Raik's [60, Thm. 1.(a)]). Suppose the projection algorithm is cyclic and has constant sets. Suppose further there is some $\epsilon>0$ s.t. $\epsilon \leq \alpha_{i}^{(n)} \leq$ $2-\epsilon$ for every index $i$ and all $n$ active for $i$. If there is some index $j \in\{1, \ldots, N\}$ s.t.

$$
C_{j} \cap \operatorname{int} \bigcap_{i \in\{1, \ldots, N\} \backslash\{j\}} C_{i} \neq \emptyset
$$

then the sequence $\left(x^{(n)}\right)$ converges in norm (in fact, linearly) to some point in $C$.

REMARK 6.9. Gubin, Polyak, and Raik's paper [60] is a cornerstone for this field and contains many original results and applications.

REMARK 6.10. The preceding examples all followed from Theorem 5.7 and the results on bounded linear regularity. Since Theorem 5.7 allows more general control, other iterations are covered as well. For example, the conclusions of the last three examples remain valid if we replace "(almost) cyclic" by "weighted." Similarly, adjusting Theorem 5.2 yields various examples on norm converge.

The following results on weak convergence follow readily from Theorem 3.20.(i).

EXAMPLE 6.11 (Browder's [17, Thm. 2] for finitely many sets). Suppose the projection algorithm is almost cyclic, unrelaxed, and has constant sets. Then the sequence $\left(x^{(n)}\right)$ converges weakly to some point in $C$.

REMARK 6.12 (cyclic projections). If in the last example "almost cyclic" is replaced by "cyclic," then one obtains the method of cyclic projections; the conclusion of the last example becomes Bregman's [16, Thm. 1]. The case when the sets $C_{i}$ do not necessarily intersect is discussed in some detail in [14]. 
EXAMPLE 6.13 (Crombez's [38, Thm. 3]). Suppose the projection algorithm is weighted and has constant sets. Suppose further the relaxation parameters and weights satisfy $0<$ $\alpha_{i}^{(n)} \equiv \alpha_{i}<2$ and $0<\lambda_{i}^{(n)} \equiv \lambda_{i}$ for every index $i$ and all $n$. Then the sequence $\left(x^{(n)}\right)$ converges weakly to some point in $C$.

REMARK 6.14. Crombez [38] assumed in addition that one of the sets is the entire space (which has the identity as projection).

\section{Consideration of remotest sets control.}

EXAMPLE 6.15 (Gubin, Polyak, and Raik's [60, Thm. 1.(a)] for finitely many sets). Suppose the projection algorithm has remotest set control and constant sets. Suppose further there is some $\epsilon>0$ s.t. $\epsilon \leq \alpha_{i}^{(n)} \leq 2-\epsilon$ for every index $i$ and all $n$ active for $i$. If there is some index $j \in\{1, \ldots, N\}$ s.t.

$$
C_{j} \cap \operatorname{int} \bigcap_{i \in\{1, \ldots, N\} \backslash\{j\}} C_{i} \neq \emptyset
$$

then the sequence $\left(x^{(n)}\right)$ converges linearly to some point in $C$.

Proof. The projection algorithm is linearly focusing and the $N$-tuple $\left(C_{1}, \ldots, C_{N}\right)$ is boundedly linearly regular (Corollary 5.14 and Remark 5.15). The result follows from Theorem 5.8.

EXAMPLE 6.16 (Bregman's [16, Thm. 2] for finitely many sets). Suppose the projection algorithm is unrelaxed and has remotest set control and constant sets. Then the sequence $\left(x^{(n)}\right)$ converges weakly to some point in $C$.

Proof. It is immediate from Theorem 4.26.(ii).

\section{Subspaces.}

EXAMPLE 6.17 (Browder's [17, Cor. to Thm. 3]). Suppose the projection algorithm is almost cyclic, unrelaxed, and has constant sets that are closed subspaces. If

$$
C_{1}^{\perp}+\cdots+C_{N}^{\perp}
$$

is closed, then the sequence $\left(x^{(n)}\right)$ converges linearly.

Proof. Combine Theorems 5.7 and 5.19.

EXAMPLE 6.18 (a remark on Smith, Solmon, and Wagner's [94, Thm. 2.2]). Suppose the projection algorithm is cyclic, unrelaxed, and has constant sets that are closed subspaces. If the angle between

$$
C_{i} \text { and } C_{i+1} \cap \cdots \cap C_{N}
$$

is positive for every index $i \in\{1, \ldots, N-1\}$, then the sequence $\left(x^{(n)}\right)$ converges linearly.

Proof. Combine Theorems 5.7 and 5.11 and Proposition 5.16.

REMARKS 6.19.

- In the last two examples, the two quite different looking hypotheses on the subspaces turned out to be special instances of bounded linear regularity. This, together with Theorem 5.7, explained linear convergence.

- It follows from Amemiya and Ando's work [5] that the limits of the sequences of the two previous examples equal

$$
P_{C} x^{(0)} \text {. }
$$

- The grandfather of these results on subspaces is the following. 
Suppose the projection algorithm is cyclic, unrelaxed, and has constant sets that are closed subspaces. Then the sequence $\left(x^{(n)}\right)$ converges in norm to $P_{C} x^{(0)}$.

The 2-subspace version is due to von Neumann [103, Thm. 13.7]; Halperin [61, Thm. 1] proved the $N$-subspace version. The reader will note that there is no hypothesis on the subspaces (and, however, no conclusion on the rate of convergence). Since bounded regularity and linear regularity of an $\mathrm{N}$-tuple of subspaces are the same (Theorem 5.19), our framework is incapable of recapturing the von Neumann/Halperin result. For applications, though, one is often interested in linear convergence results. Those follow under additional hypotheses that imply regularity (see the last two examples) and are thus covered by our framework. The best and most complete reference on the von Neumann/Halperin framework and its impressive applications is Deutsch's survey article [44]; see also Deutsch and Hundal's recent [45].

- Although mathematically intriguing, controls that are different from intermittent or remotest set control seem to be of little use for applications; consider, for example, two closed subspaces with closed sum and with intersection $\{0\}$. A singular unrelaxed projection algorithm for these two sets converges linearly whenever its control is intermittent or considers remotest sets (cf. Theorems 5.7 and 5.8). However, if we consider, for example, the random control version where we project onto the first subspace whenever $n$ is a power of 2 , and onto the second subspace otherwise, then the resulting sequence $\left(x^{(n)}\right)$ is not linearly convergent.

Hyperplanes. Hyperplanes play an important role in applications for two reasons. First, the solution of a system of linear equations is nothing but the intersection of the corresponding hyperplanes. Second, projections onto hyperplanes can be calculated easily. In fact, if a hyperplane $C_{i}$ is given by

$$
C_{i}=\left\{x \in X:\left\langle a_{i}, x\right\rangle=b_{i}\right\}
$$

for some $a_{i} \in X \backslash\{0\}$ and $b_{i} \in \mathbb{R}$, then, for every $x \in X$,

$$
P_{i} x=P_{C_{i}} x=x-\frac{\left(\left\langle a_{i}, x\right\rangle-b_{i}\right)}{\left\|a_{i}\right\|^{2}} a_{i} \text { and } d\left(x, C_{i}\right)=\frac{\left|\left\langle a_{i}, x\right\rangle-b_{i}\right|}{\left\|a_{i}\right\|} .
$$

\section{Intermittent control.}

EXAMPLE 6.20. Suppose the projection algorithm is intermittent and has constant sets that are hyperplanes. Suppose further there is some $\epsilon>0$ s.t. $\epsilon \leq \alpha_{i}^{(n)} \leq 2-\epsilon$ and $\epsilon \leq \lambda_{i}^{(n)}$ for all large $n$ and every index $i$ active at $n$. Then the sequence converges linearly to some point in $C$ with a rate independent of the starting point.

Proof. Combine Theorem 5.7 and Corollary 5.22.

The following special cases of the last example are well known.

EXAMPLE 6.21 (Herman, Lent, and Lutz's [64, Cor. 1], Trummer's [97, Thm. 5]). Suppose $X$ is finite dimensional and the projection algorithm is cyclic and has constant sets that are hyperplanes. Suppose further there is some $\epsilon>0$ s.t. $\epsilon \leq \alpha_{i}^{(n)} \leq 2-\epsilon$ for all large $n$ and every index $i$ active at $n$. Then the sequence $\left(x^{(n)}\right)$ converges linearly to some point in $C$ with a rate independent of the starting point.

EXAMPLE 6.22 (Kaczmarz [71], Gordon, Bender, and Herman [59]). Suppose $X$ is finite dimensional and the projection algorithm is cyclic, unrelaxed, and has constant sets that are hyperplanes. Then the sequence $\left(x^{(n)}\right)$ converges linearly to some point in $C$ with a rate independent of the starting point. 
REMARKS 6.23.

- The precursor of these results is certainly the last example, which was discovered by Kaczmarz as early as 1937.

- Kaczmarz's method is well understood even in the infeasible case; we refer the interested reader to Tanabe's [96] and Trummer's [97, 99].

- The iteration described in Example 6.21 is also known as "ART" (algebraic reconstruction technique).

EXAMPLE 6.24 (Trummer's [97, first part of Thm. 1]). Suppose $X$ is finite dimensional and the projection algorithm is cyclic and has constant sets that are hyperplanes. If $v_{n}$ is defined as in Theorem 5.2 and $\sum_{n} v_{n}=+\infty$, then the sequence $\left(x^{(n)}\right)$ converges in norm to some point in $C$.

Proof. It is immediate from Theorem 5.2.

REMARKS 6.25.

- Trummer also investigated the infeasible case; see [97, 99].

- Using Theorem 5.2, we can similarly recapture Trummer's [97, second part of Thm. 1], where he describes an iteration that yields a nonnegative solution (assuming there exists at least one).

REMARK 6.26. Herman et al. [65] used block control variants of Example 6.20 for image reconstruction. Their algorithms are based on a (more matrix-theoretic) framework by Eggermont, Herman, and Lent [49].

\section{Weighted control.}

EXAMPLE 6.27 (Trummer's [98, Thm. 8]). Suppose $X$ is finite dimensional and the projection algorithm is weighted, unrelaxed, and has constant sets that are hyperplanes $C_{i}=$ $\left\{x \in X:\left\langle a_{i}, x\right\rangle=b_{i}\right\}$. If the weights are given by

$$
\lambda_{i}^{(n)} \equiv \frac{\left\|a_{i}\right\|^{2}}{\sum_{j=1}^{N}\left\|a_{j}\right\|^{2}},
$$

then the sequence $\left(x^{(n)}\right)$ converges linearly to some point in $C$.

Proof. The control is 1-intermittent; thus, the result follows from Example 6.20.

REMARK 6.28. Trummer even allowed infeasible systems and identified the limit; see [98].

THEOREM 6.29. Suppose the projection algorithm is weighted and has constant sets that are hyperplanes $C_{i}=\left\{x \in X:\left\langle a_{i}, x\right\rangle=b_{i}\right\}$. Suppose further there exists a subsequence $\left(n^{\prime}\right)$ of $(n)$ and some $\epsilon>0$ s.t.

$$
\epsilon \leq \alpha_{i}^{\left(n^{\prime}\right)}, \lambda_{i}^{\left(n^{\prime}\right)} \text { for all } n^{\prime} \text { and every index } i
$$

If $\operatorname{span}\left\{a_{1}, \ldots, a_{N}\right\}$ is at least two dimensional, then the sequence $\left(x^{(n)}\right)$ converges in norm to some point in $C$.

Proof. Without loss of generality, we assume $\left\|a_{i}\right\|=1$ for every index $i$. Fix $x \in C$. Then, by Lemma 3.2.(i),

$$
\left\|x^{(n)}-x\right\|^{2}-\left\|x^{(n+1)}-x\right\|^{2} \geq \sum_{i<j} \lambda_{i}^{(n)} \lambda_{j}^{(n)} \alpha_{i}^{(n)} \alpha_{j}^{(n)}\left\|P_{i} x^{(n)}-P_{j} x^{(n)}\right\|^{2}
$$

for all $n \geq 0$. Summing over $n$ and remembering that each set $C_{i}$ is a hyperplane, we obtain a convergent series whose general term

$$
\sum_{i<j} \lambda_{i}^{(n)} \lambda_{j}^{(n)} \alpha_{i}^{(n)} \alpha_{j}^{(n)}\left\|\left(\left\langle a_{j}, x^{(n)}\right\rangle-b_{j}\right) a_{j}-\left(\left\langle a_{i}, x^{(n)}\right\rangle-b_{i}\right) a_{i}\right\|^{2}
$$


tends to 0 . Hence, along the subsequence $\left(x^{\left(n^{\prime}\right)}\right)$, we have

$$
\left(\left\langle a_{i}, x^{\left(n^{\prime}\right)}\right\rangle-b_{i}\right) a_{i}-\left(\left\langle a_{j}, x^{\left(n^{\prime}\right)}\right\rangle-b_{j}\right) a_{j} \longrightarrow 0 \text { whenever } i \neq j .
$$

Now fix any index $i$ and obtain another index $j \neq i$ s.t. $\left\{a_{i}, a_{j}\right\}$ are linearly independent. Then, by $(*)$,

$$
\left\langle a_{i}, x^{\left(n^{\prime}\right)}\right\rangle-b_{i} \longrightarrow 0 .
$$

Thus $d\left(x^{\left(n^{\prime}\right)}, C_{i}\right)=\left|\left\langle a_{i}, x^{\left(n^{\prime}\right)}\right\rangle-b_{i}\right| \longrightarrow 0$. Since $i$ has been chosen arbitrarily, we conclude that

$$
\max \left\{d\left(x^{\left(n^{\prime}\right)}, C_{i}\right): i=1 \ldots N\right\} \longrightarrow 0,
$$

and further, by linear regularity of $\left(C_{1}, \ldots, C_{N}\right)$ (Corollary 5.22),

$$
d\left(x^{\left(n^{\prime}\right)}, C\right) \longrightarrow 0 .
$$

The result follows from Corollary 3.3.(ii).

The following classical example is now obvious.

EXAMPLE 6.30 (Cimmino's method [29] in Hilbert space). Suppose the projection algorithm is weighted and has constant sets that are hyperplanes $C_{i}=\left\{x \in X:\left\langle a_{i}, x\right\rangle=b_{i}\right\}$. Suppose further the relaxation parameters and weights satisfy

$$
\alpha_{i}^{(n)} \equiv 2, \quad \lambda_{i}^{(n)} \equiv \lambda_{i}>0
$$

for all $n \geq 0$ and every index $i$. If $\operatorname{span}\left\{a_{1}, \ldots, a_{N}\right\}$ is at least two dimensional, then the sequence $\left(x^{(n)}\right)$ converges in norm to some point in $C$.

REMARKS 6.31.

- For Euclidean spaces, the last example was known to Cimmino as far back as 1938. His method has a nice geometric interpretation: one obtains $x^{(n+1)}$ from $x^{(n)}$ by reflecting $x^{(n)}$ in all $N$ hyperplanes and then taking a weighted average.

- As Example 3.6 shows, the assumption on span $\left\{a_{1}, \ldots, a_{N}\right\}$ is essential.

- Due to their parallelizability, Cimmino's and related methods with weighted control are currently used with great success; see Censor's survey article [23].

We present a variation of Cimmino's method that includes a method suggested by Ansorge.

EXAMPLE 6.32 (a generalization of Ansorge's method [6]). Suppose $X$ is finite dimensional and the projection algorithm has constant sets where $C_{N}=X$. Suppose further that

$$
\alpha_{N}^{(n)} \equiv 1, \quad \lambda_{N}^{(n)} \equiv \lambda_{N}>0, \quad \alpha_{1}^{(n)} \equiv \cdots \equiv \alpha_{N-1}^{(n)} \equiv 2,
$$

and

$$
\lambda_{i}^{(n)}:= \begin{cases}\frac{\left(1-\lambda_{N}\right) f\left(d\left(x^{(n)}, C_{i}\right)\right)}{\sum_{j=1}^{N} f\left(d\left(x^{(n)}, C_{j}\right)\right)} & \text { if } x^{(n)} \notin C, \\ \frac{1-\lambda_{N}}{N-1} & \text { otherwise }\end{cases}
$$

for some strictly increasing continuous function $f:[0,+\infty[\rightarrow[0,+\infty[$ with $f(0)=0$. Then the sequence $\left(x^{(n)}\right)$ converges in norm to some point in $C$.

Proof. Clearly, the projection algorithm is strongly focusing and considers remotest sets. The $N$-tuple $\left(C_{1}, \ldots, C_{N}\right)$ is boundedly regular (Proposition 5.4.(iii)). Suppose that $\left(i^{(n)}\right)$ is a sequence of active remotest indices. Then $i^{(n)} \in\{1, \ldots, N-1\}$ and

$$
\mu_{i^{(n)}}^{(n)}=\lambda_{i^{(n)}}^{(n)} 2 \lambda_{N}
$$


If $\sum_{n} \lambda_{i^{(n)}}^{(n)}=+\infty$, then we are done by Theorem 5.3. Otherwise $\sum_{n} \lambda_{i^{(n)}}^{(n)}<+\infty$ and hence $\lambda_{i^{(n)}}^{(n)} \longrightarrow 0$. Now $\left(x^{(n)}\right)$ is bounded and $f$ is continuous; thus $\left(\sum_{j} f\left(d\left(x^{(n)}, C_{j}\right)\right)\right)_{n}$ is a bounded sequence. Consequently,

$$
f\left(d\left(x^{(n)}, C_{i}\right)\right) \leq f\left(d\left(x^{(n)}, C_{i^{(n)}}\right)\right) \longrightarrow 0 \text { for every index } i,
$$

which implies that

$$
\max \left\{d\left(x^{(n)}, C_{i}\right): i=1 \ldots N\right\} \longrightarrow 0 .
$$

Because of the bounded regularity of $\left(C_{1}, \ldots, C_{N}\right)$, we get $d\left(x^{(n)}, C\right) \longrightarrow 0$; now Corollary 3.3.(ii) completes the proof.

REMARK 6.33. Ansorge's method [6] arises when the sets $C_{1}, \ldots, C_{N-1}$ are hyperplanes and $f=|\cdot|^{\gamma}$ for $\gamma>0$.

Halfspaces. Halfspaces play an important role for essentially the same reasons hyperplanes do: their intersection describes the solution of the corresponding system of linear inequalities (this problem is also referred to as the linear feasibility problem) and the projections are easy to calculate. Indeed, if a halfspace $C_{i}$ is given by

$$
C_{i}=\left\{x \in X:\left\langle a_{i}, x\right\rangle \leq b_{i}\right\}
$$

for some $a_{i} \in X \backslash\{0\}$ and $b_{i} \in \mathbb{R}$, then, for every $x \in X$,

$$
P_{i} x=P_{C_{i}} x=x-\frac{\left(\left\langle a_{i}, x\right\rangle-b_{i}\right)^{+}}{\left\|a_{i}\right\|^{2}} a_{i} \text { and } d\left(x, C_{i}\right)=\frac{\left(\left\langle a_{i}, x\right\rangle-b_{i}\right)^{+}}{\left\|a_{i}\right\|} .
$$

Some of the algorithms for finding a solution of the linear feasibility problem discussed below have been used with great success in radiation therapy treatment planning; we refer the reader to Censor, Altschuler, and Powlis's interesting survey article [25].

\section{Intermittent control.}

EXAMPLE 6.34. Suppose the projection algorithm is intermittent and has constant sets that are halfspaces. Suppose further there is some $\epsilon>0$ s.t. $\epsilon \leq \alpha_{i}^{(n)} \leq 2-\epsilon$ and $\epsilon \leq \lambda_{i}^{(n)}$ for all large $n$ and every index $i$ active at $n$. Then the sequence $\left(x^{(n)}\right)$ converges linearly to some point in $C$ with a rate independent of the starting point.

Proof. Combine Theorem 5.7 and Fact 5.23.

We deduce readily the next two examples.

EXAMPLE 6.35 (Gubin, Polyak, and Raik's [60, Thm. 1.(d)], Herman, Lent, and Lutz's [64, Thm. 1]). Suppose the projection algorithm is cyclic and has constant sets that are halfspaces. Suppose further there is some $\epsilon>0$ s.t. $\epsilon \leq \alpha_{i}^{(n)} \leq 2-\epsilon$ for all large $n$ and every index $i$ active at $n$. Then the sequence $\left(x^{(n)}\right)$ converges in norm to some point in $C$.

REMARKS 6.36. By Example 6.34, the rate of convergence of the sequence $\left(x^{(n)}\right)$ is actually linear and independent of the starting point. Herman, Lent, and Lutz assumed additionally that $X$ is finite dimensional. Mandel [78, Thm. 3.1] offered an upper bound for the rate of convergence for the case when $X$ is finite dimensional and $0<\alpha_{i}^{(n)} \equiv \alpha<2$.

EXAMPLE 6.37 (Censor, Altschuler, and Powlis's [25, Alg. 3]). Suppose the projection algorithm considers only blocks and has constant sets that are hyperplanes. Suppose further there is some $\epsilon>0$ s.t. $\epsilon \leq \alpha_{i}^{(n)} \leq 2-\epsilon$ for all $n \geq 0$ and every index $i$. Suppose finally that for every index $i$, there is some $\lambda_{i}>0$ s.t. $\lambda_{i}^{(n)}=\lambda_{i}$ for all $n$ active for $i$. Then the sequence $\left(x^{(n)}\right)$ converges linearly to some point in $C$ with a rate independent of the starting point.

REMARKS 6.38. Censor, Altschuler, and Powlis [25] offered no results on convergence; however, Aharoni and Censor's [3, Thm. 1] yields norm convergence of $\left(x^{(n)}\right)$ in Euclidean spaces. We thus add two features. First, we remove the restriction on finite dimensionality. Second, we establish linear convergence. 
Weighted control. The following two examples are also consequences of Example 6.34.

EXAMPLE 6.39 (Eremin's [52, Cor. to Thm. 1.2]). Suppose the projection algorithm is weighted and has constant sets that are halfspaces. Suppose further the relaxation parameters and weights satisfy

$$
0<\alpha_{i}^{(n)} \equiv \alpha_{i}<2, \quad 0<\lambda_{i}^{(n)} \equiv \lambda_{i}, \quad \alpha_{i} \lambda_{i}<\frac{2}{N}
$$

for every index $i$ and all $n$. Then the sequence $\left(x^{(n)}\right)$ converges linearly to some point in $C$ with a rate independent of the starting point.

EXAMPLE 6.40 (the feasible case of De Pierro and Iusem's [39, Lem. 8]). Suppose $X$ is finite dimensional and the projection algorithm is weighted and has constant sets that are halfspaces. Suppose further there is some $\epsilon>0$ s.t. $\epsilon \leq \alpha_{i}^{(n)} \equiv \alpha^{(n)} \leq 2-\epsilon, 0<\lambda_{i}^{(n)} \equiv \lambda_{i}$ for every index $i$ and all $n$. Then the sequence $\left(x^{(n)}\right)$ converges in norm to some point in $C$.

REMARKS 6.41. In the last example, the rate of convergence of the sequence $\left(x^{(n)}\right)$ is actually linear and independent of the starting point. For a slightly more restrictive scheme, De Pierro and Iusem could also identify the limit of $\left(x^{(n)}\right)$ in the infeasible case as a least squares solution; see [39].

\section{Consideration of remotest sets control.}

EXAMPLE 6.42. Suppose the projection algorithm considers remotest sets and has constant sets that are halfspaces. Suppose further that $\left(i^{(n)}\right)$ is a sequence of active remotest indices. If $\underline{\lim }_{n} \mu_{i^{(n)}}^{(n)}>0$, then the sequence $\left(x^{(n)}\right)$ converges linearly to some point in $C$ with a rate independent of the starting point.

Proof. Combine Theorem 5.8 and Fact 5.23.

EXAMPLE 6.43 (Gubin, Polyak, and Raik's [60, Thm. 1.(d)]). Suppose the projection algorithm has remotest set control and constant sets that are halfspaces. If there is some $\epsilon>0$ s.t. $\epsilon \leq \alpha_{i}^{(n)} \leq 2-\epsilon$ for all $n$ and every index $i$ active at $n$, then the sequence $\left(x^{(n)}\right)$ converges linearly to some point in $C$ with a rate independent of the starting point.

Proof. We have for any index $i$ active at $n$,

$$
\mu_{i}^{(n)}=\lambda_{i}^{(n)} \alpha_{i}^{(n)}\left(2-\sum_{j=1}^{N} \lambda_{j}^{(n)} \alpha_{j}^{(n)}\right)=\alpha_{i}^{(n)}\left(2-\alpha_{i}^{(n)}\right) \geq \epsilon^{2}
$$

the result thus follows from the previous example.

The basic result in this subsection is due to Agmon and Motzkin and Schoenberg. It dates back to as early as 1954 .

EXAMPLE 6.44 (Agmon's [1, Thm. 3], Motzkin and Schoenberg's [83, Case 1 in Thm. 1 and Thm. 2]). Suppose $X$ is finite dimensional and the projection algorithm has remotest set control and constant sets that are halfspaces. If $0<\alpha_{i}^{(n)} \equiv \alpha<2$ for all $n$ and every index $i$ active at $n$, then the sequence $\left(x^{(n)}\right)$ converges in norm to some point in $C$.

Proof. This is a special case of the preceding example.

REMARKS 6.45.

- While Agmon considered only the case when $\alpha=1$, he already obtained linear convergence of $\left(x^{(n)}\right)$ with a rate independent of the starting point.

- Motzkin and Schoenberg did not establish linear convergence; they discussed, however, the case when $\alpha=2$.

- It follows from Example 6.43 that the rate of convergence is linear and independent of the starting point. Again, Mandel provided an upper bound for the rate; see [78, Thm. 2.2].

THEOREM 6.46. Suppose $N \geq 2$, the projection algorithm has constant sets that are halfspaces $C_{i}=\left\{x \in X:\left\langle a_{i}, x\right\rangle \leq b_{i}\right\}$, and violated constraints correspond exactly to active 
indices; i.e., if $x^{(n)} \notin C$, then

$$
x^{(n)} \notin C_{i} \Longleftrightarrow i \in I^{(n)} .
$$

Suppose further that there is some $\epsilon>0$ s.t. $\epsilon \leq \alpha_{i}^{(n)}, \lambda_{i}^{(n)}$ for all large $n$ and every index $i$ active at $n$ and that

$$
I^{(n)}=\{i\} \text { implies } \alpha_{i}^{(n)} \leq 2-\epsilon .
$$

Suppose finally that if $i$ and $j$ are two distinct indices, then

$$
\left\{\begin{array}{l}
\text { either }\left\{a_{i}, a_{j}\right\} \text { is linearly independent } \\
\text { or } X \backslash C_{i} \subseteq C_{j} \text { (equivalently, } X \backslash C_{j} \subseteq C_{i} \text { ), } \\
\text { but never both. }
\end{array}\right.
$$

Then the sequence $\left(x^{(n)}\right)$ converges in norm to some point in $C$.

Proof. We assume without loss that $\left\|a_{i}\right\|=1$ for every index $i$ and that $x^{(n)} \notin C$ for all $n$ (otherwise, the projection algorithm becomes constant anyway). Clearly, the projection algorithm is linearly focusing and considers remotest sets, so let $\left(i^{(n)}\right)$ be a sequence of active remotest indices. Since the $N$-tuple $\left(C_{1}, \ldots, C_{N}\right)$ is linearly regular (Fact 5.23), we can also assume that $\sum_{n} \mu_{i^{(n)}}^{(n)}<+\infty$ (otherwise, we are done by Theorem 5.3). Hence

$$
\mu_{i^{(n)}}^{(n)}=\lambda_{i^{(n)}}^{(n)} \alpha_{i^{(n)}}^{(n)}\left(2-\sum_{j=1}^{N} \lambda_{j}^{(n)} \alpha_{j}^{(n)}\right) \longrightarrow 0 .
$$

Claim 1:

$$
I^{(n)} \text { is not a singleton for all large } n \text {. }
$$

Otherwise, there would be a subsequence $\left(n^{\prime}\right)$ of $(n)$ s.t. $I^{\left(n^{\prime}\right)}=\left\{i^{\left(n^{\prime}\right)}\right\}$. On the other hand, $\mu_{i^{\left(n^{\prime}\right)}}^{\left(n^{\prime}\right)}=1 \alpha_{i^{\left(n^{\prime}\right)}}^{\left(n^{\prime}\right)}\left(2-1 \alpha_{i^{\left(n^{\prime}\right)}}^{\left(n^{\prime}\right)}\right) \geq \epsilon^{2}$, which would contradict $\mu_{i^{(n)}}^{(n)} \longrightarrow 0$. Hence Claim 1 is verified.

By Claim 1, we can find a subsequence $\left(n^{\prime}\right)$ of $(n)$ and two distinct indices $i, j$ s.t.

$$
i^{\left(n^{\prime}\right)} \equiv i \text { and }\{i, j\} \subseteq I^{\left(n^{\prime}\right)} \text { for all } n^{\prime} .
$$

Claim 2:

$$
\left\{a_{i}, a_{j}\right\} \text { is linearly independent. }
$$

Otherwise, $X \backslash C_{i} \subseteq C_{j}$. Since $x^{\left(n^{\prime}\right)} \notin C_{i}$, we would conclude $x^{\left(n^{\prime}\right)} \in C_{j}$, which would contradict $j \in I^{\left(n^{\prime}\right)}$. Thus Claim 2 holds.

Similarly to the proof of Theorem 6.29 , we get

$$
P_{i} x^{\left(n^{\prime}\right)}-P_{j} x^{\left(n^{\prime}\right)} \longrightarrow 0
$$

or

$$
\left(\left\langle a_{i}, x^{\left(n^{\prime}\right)}\right\rangle-b_{i}\right) a_{i}-\left(\left\langle a_{j}, x^{\left(n^{\prime}\right)}\right\rangle-b_{j}\right) a_{j} \longrightarrow 0 .
$$

Now $\left\langle a_{i}, x^{\left(n^{\prime}\right)}\right\rangle-b_{i}=d\left(x^{\left(n^{\prime}\right)}, C_{i}\right)$ and $\left\langle a_{j}, x^{\left(n^{\prime}\right)}\right\rangle-b_{j}=d\left(x^{\left(n^{\prime}\right)}, C_{j}\right)$; hence Claim 2 implies in particular that $d\left(x^{\left(n^{\prime}\right)}, C_{i}\right) \longrightarrow 0$, or, recalling that $i^{\left(n^{\prime}\right)} \equiv i$,

$$
\max \left\{d\left(x^{\left(n^{\prime}\right)}, C_{l}\right): l=1 \ldots N\right\} \longrightarrow 0 .
$$

The linear regularity of $\left(C_{1}, \ldots, C_{N}\right)$ yields $d\left(x^{\left(n^{\prime}\right)}, C\right) \rightarrow 0$. Now apply Corollary 3.3.(ii).

EXAMPLE 6.47 (Censor and Elfving's framework [26, Alg. 1]). Suppose $X$ is finite dimensional, $N \geq 2$, and the projection algorithm has constant sets that are halfspaces. Define $I_{\text {out }}^{(n)}:=\left\{i \in\{1, \ldots, N\}: x^{(n)} \notin C_{i}\right\}$ for all $n \geq 0$, and let $m_{1}, \ldots, m_{N}>0$ be given constants with $\sum_{i=1}^{N} m_{i}=1$. Suppose further the relaxation parameters and weights are chosen according to the following cases. 
0: $I_{\text {out }}^{(n)}$ is empty. Then choose the relaxation parameters and weights as you wish (the projection algorithm becomes constant anyway).

1: $I_{\text {out }}^{(n)}=\left\{i^{(n)}\right\}$ is a singleton. Then set

$$
\alpha_{i}^{(n)}: \equiv 2 m_{i^{(n)}} \text { and } I^{(n)}:=\left\{i^{(n)}\right\} .
$$

2: $I_{\text {out }}^{(n)}$ contains at least two indices. Then set

$$
\alpha_{i}^{(n)}: \equiv 2 \text { and } \lambda_{i}^{(n)}= \begin{cases}0 & \text { if } x^{(n)} \in C_{i}, \\ \frac{m_{i}}{\sum_{j: j \notin C_{j}} m_{j}} & \text { otherwise. }\end{cases}
$$

Suppose finally that if $i$ and $j$ are two distinct indices, then

$$
\left\{\begin{array}{l}
\text { either }\left\{a_{i}, a_{j}\right\} \text { is linearly independent } \\
\text { or } X \backslash C_{i} \subseteq C_{j} \text { (equivalently, } X \backslash C_{j} \subseteq C_{i} \text { ), } \\
\text { but never both. }
\end{array}\right.
$$

Then the sequence $\left(x^{(n)}\right)$ converges in norm to some point in $C$.

Proof. This is a special case of the previous example.

REMARKS 6.48.

- Censor and Elfving also investigated an iteration [26, Alg. 2] that is more general than the iteration in Example 6.47. Their method of proof is more matrix theoretic and is quite different from ours.

- They claimed that the last example does not need the hypothesis $(*)$. This is, however, false since otherwise a suitable modification of Example 3.6 would yield a counterexample.

- It is possible to recapture Cimmino's method (Example 6.30) for pairwise distinct hyperplanes by describing each hyperplane $\left\{x \in X:\left\langle a_{i}, x\right\rangle=b_{i}\right\}$ by the corresponding two halfspaces $\left\{x \in X:\left\langle a_{i}, x\right\rangle \leq b_{i}\right\},\left\{x \in X:\left\langle-a_{i}, x\right\rangle \leq-b_{i}\right\}$ and then applying the previous example. This nice observation is due to Censor and Elfving. The assumption that the hyperplanes are pairwise distinct is not really severe; it merely means that "each hyperplane should be counted only once."

REMARK 6.49. More algorithms for solving the linear feasibility problem are given in $\S 7$.

Polyhedra. The class of polyhedra is large: it contains the class of halfspaces, the class of hyperplanes, and the class of finite-co-dimensional affine subspaces. It is generally not easy to calculate projections onto polyhedra; there are, however, besides the examples discussed in the previous subsections, two additional important exceptions-hyperslabs and the finitedimensional positive cone.

A hyperslab $C_{i}$ is given by

$$
C_{i}=\left\{x \in X: c_{i} \leq\left\langle a_{i}, x\right\rangle \leq b_{i}\right\}
$$

for some $a_{i} \in X \backslash\{0\}$ and two real numbers $c_{i} \leq b_{i}$. Then, for every $x$,

$$
P_{i} x=P_{C_{i}} x=x-\frac{\left(\left\langle a_{i}, x\right\rangle-b_{i}\right)^{+}-\left(c_{i}-\left\langle a_{i}, x\right\rangle\right)^{+}}{\left\|a_{i}\right\|^{2}} a_{i}
$$

and

$$
d\left(x, C_{i}\right)=\frac{\left|\left(\left\langle a_{i}, x\right\rangle-b_{i}\right)^{+}-\left(c_{i}-\left\langle a_{i}, x\right\rangle\right)^{+}\right|}{\left\|a_{i}\right\|} .
$$


The positive cone in $X:=\mathbb{R}^{d}$ is denoted $X^{+}$and is given by $X^{+}=\left\{x \in X: x_{i} \geq\right.$ 0 for $i=1, \ldots, d\}$. Its projection is given by $x=\left(x_{i}\right)_{i=1}^{d} \longmapsto x^{+}:=\left(x_{i}^{+}\right)_{i=1}^{d}$ for every $x \in X$.

EXAMPLE 6.50 (Censor, Altschuler, and Powlis's [25, Alg. 4]). Suppose $X$ is finite dimensional and the projection algorithm has constant sets that are hyperslabs except $C_{N}=$ $X^{+}$. Suppose further the projection algorithm considers only blocks, where the number of blocks is $M$ and $J_{M}=\{N\}$. If there is some $\epsilon>0$ s.t. $\epsilon \leq \alpha_{i}^{(n)} \leq 2-\epsilon$ and $\epsilon \leq \lambda_{i}^{(n)}$ for all $n$ and every index $i$ active at $n$, then the sequence $\left(x^{(n)}\right)$ converges linearly to some point in $C$ with a rate independent of the starting point.

Proof. By Corollary 5.26, the $N$-tuple $\left(C_{1}, \ldots, C_{N}\right)$ is linearly regular. Now apply Theorem 5.7.

REMARK 6.51. Again, Aharoni and Censor's [3, Thm. 1] guarantees norm convergence. We obtain in addition linear convergence.

\section{Harvest time III: Subgradient algorithms.}

Theory. We return to the setting of $\S 4$, where we defined projection algorithms. Loosely speaking, "a projection algorithm that for at least one index $i$ chooses its supersets $C_{i}^{(n)}$ of $C_{i}$ to be halfspaces constructed from subgradients of a fixed convex function" is called a subgradient algorithm. Before we make this "construction" precise, we collect some basic facts on subgradients.

Definition 7.1. Suppose $f: X \longrightarrow \mathbb{R}$ is a convex function. Given a point $x_{0} \in X$, the set

$$
\left\{x^{*} \in X:\left\langle x^{*}, x-x_{0}\right\rangle \leq f(x)-f\left(x_{0}\right) \text { for all } x \in X\right\}
$$

is called the subdifferential of $f$ at $x_{0}$ and is denoted $\partial f\left(x_{0}\right)$. The elements of $\partial f\left(x_{0}\right)$ are called subgradients of $f$ at $x_{0}$. If $\partial f\left(x_{0}\right)$ is nonempty, then $f$ is said to be subdifferentiable at $x_{0}$.

The importance of this concept stems from the easy-to-verify fact that

$$
x_{0} \text { is a minimizer of } f \Longleftrightarrow 0 \in \partial f\left(x_{0}\right) \text {. }
$$

Deeper are the following facts: for proofs see, for example, Ekeland and Temam's [50, Chap. I: Cor. 2.5, Prop. 5.3, Prop. 5.2, and Cor. 2.3].

FACTS 7.2. Suppose $f: X \longrightarrow \mathbb{R}$ is a convex function and $x_{0} \in X$. Then

(i) $f$ is continuous at $x_{0}$ and $\partial f\left(x_{0}\right)$ is a singleton if and only if $f$ is lower semicontinuous and Gâteaux differentiable at $x_{0}$. In this case, the unique subgradient of $f$ at $x_{0}$ coincides with the Gâteaux derivative of $f$ at $x_{0}$.

(ii) If $f$ is continuous at $x_{0}$, then $f$ is subdifferentiable at $x_{0}$.

(iii) If $X$ is finite dimensional, then $f$ is continuous and subdifferentiable everywhere.

LEMMA 7.3. Suppose $f: X \rightarrow \mathbb{R}$ is a convex function, $x_{0} \in X$, and $f$ is subdifferentiable at $x_{0}$. Suppose further $S:=\{x \in X: f(x) \leq 0\}$ is nonempty. For any $g\left(x_{0}\right) \in \partial f\left(x_{0}\right)$, define the closed convex set $H$ by

$$
H:=H\left(f, x_{0}, g\left(x_{0}\right)\right):=\left\{x \in X: f\left(x_{0}\right)+\left\langle g\left(x_{0}\right), x-x_{0}\right\rangle \leq 0\right\} .
$$

Then

(i) $H \supseteq S$. If $g\left(x_{0}\right) \neq 0$, then $H$ is a halfspace; otherwise, $H=X$.

(ii) $P_{H} x_{0}= \begin{cases}x_{0}-\frac{f\left(x_{0}\right)}{\left\|g\left(x_{0}\right)\right\|^{2}} g\left(x_{0}\right) & \text { if } f\left(x_{0}\right)>0 \\ x_{0} & \text { otherwise. }\end{cases}$ 
(iii) $d\left(x_{0}, H\right)= \begin{cases}\frac{f\left(x_{0}\right)}{\left\|g\left(x_{0}\right)\right\|} & \text { if } f\left(x_{0}\right)>0, \\ 0 & \text { otherwise. }\end{cases}$

Proof. (i): If $s \in S$, then $f\left(x_{0}\right)+\left\langle g\left(x_{0}\right), s-x_{0}\right\rangle \leq f(s) \leq 0$ and hence $s \in H$.

(ii): Use Facts 1.5.(ii) to verify the candidate for $P_{H} x_{0}$. (iii) follows immediately from (ii).

REMARK 7.4. The importance of the halfspace defined in the last lemma is explained by the following. Suppose we want to find a point in $S$; i.e., we look for a solution of the convex feasibility problem $f(x) \leq 0$. If $f\left(x_{0}\right) \leq 0$, then we are done. Otherwise $f\left(x_{0}\right)>0$. It is usually "hard" to solve $f(x)=0$ (otherwise, we would just solve); therefore, we instead consider a first-order approximation of $f$, say

$$
f(x) \approx \tilde{f}(x):=f\left(x_{0}\right)+\left\langle g\left(x_{0}\right), x-x_{0}\right\rangle \text { for some } g\left(x_{0}\right) \in \partial f\left(x_{0}\right),
$$

and solve $\tilde{f}\left(x_{0}\right)=0$, to which a solution is given by

$$
P_{H} x_{0}=x_{0}-\frac{f\left(x_{0}\right)}{\left\|g\left(x_{0}\right)\right\|^{2}} g\left(x_{0}\right) .
$$

We now give the precise definition of a subgradient algorithm.

DEFINITION 7.5. Suppose for some index $i \in\{1, \ldots, N\}$ and for all $n$ every set $C_{i}^{(n)}$ of a given projection algorithm is of the form

$$
\begin{aligned}
C_{i}^{(n)} & =H\left(f_{i}, x^{(n)}, g_{i}\left(x^{(n)}\right)\right) \\
& =\left\{x \in X: f_{i}\left(x^{(n)}\right)+\left\langle g_{i}\left(x^{(n)}\right), x-x^{(n)}\right\rangle \leq 0\right\}
\end{aligned}
$$

for some fixed convex function $f_{i}: X \rightarrow \mathbb{R}$, where $f_{i}$ is subdifferentiable at every $x^{(n)}$ and $g_{i}\left(x^{(n)}\right) \in \partial f_{i}\left(x^{(n)}\right)$. Suppose further that

$$
C_{i}=\left\{x \in X: f_{i}(x) \leq 0\right\} .
$$

Then we call this projection algorithm a subgradient algorithm. Every such index $i$ is called a subgradient index; the set of all subgradient indices is denoted $I_{\partial}$.

REMARKS 7.6.

- Subgradient algorithms and projection algorithms are closely related in the following sense.

(i) Every subgradient algorithm is a projection algorithm (by definition).

(ii) Every projection algorithm with constant sets can be viewed as a subgradient algorithm. To see this, one chooses $f_{i}:=d\left(\cdot, C_{i}\right)$ and takes into account that

$$
\partial f_{i}(x)=\partial d\left(x, C_{i}\right)= \begin{cases}\frac{x-P_{i} x}{\left\|x-P_{i} x\right\|} & \text { if } x \notin C_{i}, \\ N_{C_{i}}(x) \cap B_{X} & \text { otherwise, }\end{cases}
$$

where $N_{C_{i}}(x)=\left\{x^{*} \in X:\left\langle C_{i}-x, x^{*}\right\rangle \leq 0\right\}$ is the normal cone of $C_{i}$ at $x$.

- The aim of subgradient algorithms is to solve convex feasibility problems. For a good survey on subgradient algorithms and other methods for solving convex feasibility methods, see Censor's [22].

- The reader should be warned that our use of the term "subgradient algorithm" is not quite standard. In the literature, "subgradient algorithms" may refer to considerably more general algorithms; see, for example, Shor's [92]. 
We now provide a fairly large class of focusing subgradient algorithms to which our previous results are applicable.

THEOREM 7.7 (prototype of a focusing subgradient algorithm). Given a subgradient algorithm, suppose the subdifferentials of $f_{i}$ are nonempty and uniformly bounded on bounded sets for every index $i \in I_{\partial}$. Suppose further $\left(P_{i}^{(n)}\right)$ converges actively pointwise to $P_{i}$ for every index $i \in\{1, \ldots, N\} \backslash I_{\partial}$. Then the subgradient algorithm is focusing.

Proof. Fix an index $i \in\{1, \ldots, N\}$. Suppose $\left(x^{\left(n_{k}\right)}\right)$ is a subsequence of $\left(x^{(n)}\right)$ with $x^{\left(n_{k}\right)} \rightarrow x, x^{\left(n_{k}\right)}-P_{i}^{\left(n_{k}\right)} x^{\left(n_{k}\right)} \rightarrow 0$, and $i$ is active at $n_{k}$ for all $k$. We must show that $x \in C_{i}$. In view of Lemma 4.2, we need only consider the case when $i \in I_{\partial}$. Then, by weak lower semicontinuity of $f_{i}$,

$$
f_{i}(x) \leq \underline{\lim }_{k} f_{i}\left(x^{\left(n_{k}\right)}\right) .
$$

If $f_{i}\left(x^{\left(n_{k}\right)}\right) \leq 0$ infinitely often, then clearly $f_{i}(x) \leq 0$ and so $x \in C_{i}$. Otherwise $f_{i}\left(x^{\left(n_{k}\right)}\right)>0$ for all large $k$. Since the sequence $\left(x^{(n)}\right)$ is bounded, there is some $M>0$ s.t. the norm of every subgradient of $f_{i}$ at $x^{(n)}$ is at most $M$. Thus, by Lemma 7.3.(iii),

$$
0 \longleftarrow d\left(x^{\left(n_{k}\right)}, C_{i}^{\left(n_{k}\right)}\right)=\frac{f_{i}\left(x^{\left(n_{k}\right)}\right)}{\left\|g_{i}\left(x^{\left(n_{k}\right)}\right)\right\|} \geq \frac{f_{i}\left(x^{\left(n_{k}\right)}\right)}{M}
$$

hence $f_{i}\left(x^{\left(n_{k}\right)}\right) \longrightarrow 0$. Therefore, $f_{i}(x) \leq 0$ and $x \in C_{i}$.

The property that $\partial f_{i}$ is uniformly bounded on bounded sets is a standard assumption for theorems on subgradient algorithms; see, for example, $[52,86,28,4,40]$. We now characterize this property.

PROPOSITION 7.8 (uniform boundedness of subdifferentials on bounded sets). Suppose $f: X \longrightarrow \mathbb{R}$ is a convex function. Then the following conditions are equivalent.

(i) $f$ is bounded on bounded sets.

(ii) $f$ is (globally) Lipschitz continuous on bounded sets.

(iii) The subdifferentials of $f$ are nonempty and uniformly bounded on bounded sets.

Proof. "(i) $\Longrightarrow$ (ii)" can be found in Roberts and Varberg's [88, Proof of Thm. 41.B].

"(ii) $\Longrightarrow$ (iii)": By Facts 7.2.(ii), $f$ is subdifferentiable everywhere. It is enough to show that the subgradients of $f$ are uniformly bounded on open balls centered at 0 . So fix $r>0$ and obtain (by assumption) a Lipschitz constant for $f$ on int $r B_{X}$, say $L$. Now fix $x \in \operatorname{int} r B_{X}$ and get $s>0$ s.t. $x+s B_{X} \subseteq$ int $r B_{X}$. Pick any $x^{*} \in \partial f(x)$ and $b \in B_{X}$. Then

$$
\left\langle x^{*}, s b\right\rangle \leq f(x+s b)-f(x) \leq L s\|b\|
$$

thus $\left\|x^{*}\right\|=\sup \left\langle x^{*}, B_{X}\right\rangle \leq L$ and therefore the subgradients of $f$ are uniformly bounded on int $r B_{X}$ by $L$.

"(iii) $\Longrightarrow(\mathrm{i})$ ": It is enough to show that $f$ is bounded on $r B_{X}$ for every $r>0$. By assumption, there is some $M>0$ s.t. the norm of any subgradient of $f$ at any point in $r B_{X}$ is at most $M$. Fix $x \in r B_{X}$. On the one hand, pick $x^{*} \in \partial f(x)$. Then $\left\langle x^{*}, 0-x\right\rangle \leq f(0)-f(x)$; thus $f(x) \leq f(0)+\left\langle x^{*}, x\right\rangle \leq f(0)+M r$. Hence $f$ is bounded above on $r B_{X}$ by $f(0)+M r$. On the other hand, picking $x_{0}^{*} \in \partial f(0)$ shows similarly that $f$ is bounded below on $r B_{X}$ by $f(0)-M r$. Altogether, $f$ is bounded on $r B_{X}$ and the proof is complete.

COROLLARY 7.9. If $X$ is finite dimensional, then every convex function from $X$ to $\mathbb{R}$ is subdifferentiable everywhere and its subdifferentials are uniformly bounded on bounded sets.

Proof. By Facts 7.2.(iii), any convex function from $X$ to $\mathbb{R}$ is continuous everywhere. Since $X$ is finite dimensional, this function attains its minimum and maximum on bounded closed sets; in particular, it is bounded on bounded closed sets. The result now follows from the previous proposition. 
REMARKS 7.10.

- The last corollary implies that if $X$ is finite dimensional, then every convex function from $X$ to $\mathbb{R}$ can be used in Theorem 7.7.

- As the following example demonstrates, the assumption that $X$ is finite dimensional in the last corollary cannot be dropped. Consequently, the convex function constructed below cannot be used in Theorem 7.7.

EXAMPLE 7.11. Define the function $f$ by

$$
f: X:=\ell_{2} \longrightarrow \mathbb{R}: \mathbf{x}=\left(x_{n}\right) \longmapsto \sum_{n=1}^{\infty} n x_{n}^{2 n} .
$$

Then $f$ is everywhere finite, convex, continuous, and subdifferentiable. However, on $B_{X}$, neither is the function $f$ bounded nor are the subdifferentials of $f$ uniformly bounded.

Proof. Fix an arbitrary $\mathbf{x}=\left(x_{n}\right) \in X$. Then, on the one hand, $x_{n} \longrightarrow 0$. On the other hand, $\sqrt[n]{n} \longrightarrow 1$. Hence, eventually

$$
x_{n}^{2} \leq \frac{1}{(\sqrt[n]{n})^{3}} \Longleftrightarrow n x_{n}^{2 n} \leq \frac{1}{n^{2}}
$$

thus $f(\mathbf{x})$ is finite. Also, $f$ is, as the supremum of convex and lower semicontinuous functions

$$
f=\sup _{m} \sum_{n=1}^{m} n x_{n}^{2 n}
$$

convex and lower semicontinuous too. Therefore, $f$ is everywhere continuous (see, for example, [50, Chap. I: Cor. 2.5]) and subdifferentiable (Fact 7.2.(ii)). Choosing $\mathbf{x}=n$th unit vector in $X$ shows that

$$
\sup f\left(B_{X}\right) \geq f(\mathbf{x})=n ;
$$

thus $f$ is unbounded on $B_{X}$. The proof of "(iii) $\Longrightarrow(\mathrm{i})$ " in the last proposition shows that the subgradients of $f$ are not uniformly bounded on $B_{X}$.

Under a Slater-type constraint qualification, we even obtain linearly focusing subgradient algorithms.

THEOREM 7.12 (prototype of a linearly focusing subgradient algorithm). Given a subgradient algorithm, suppose that there is some Slater point $\hat{x} \in X$ s.t.

$$
f_{i}(\hat{x})<0
$$

and that the subdifferentials of $f_{i}$ are nonempty and uniformly bounded on bounded sets for every subgradient index $i \in I_{\partial}$. Suppose further there is some $\beta>0$ s.t.

$$
\beta d\left(x^{(n)}, C_{i}\right) \leq d\left(x^{(n)}, C_{i}^{(n)}\right)
$$

for every index $i \in\{1, \ldots, N\} \backslash I_{\partial}$ and all large $n$ active for $i$. Then the subgradient algorithm is linearly focusing.

Proof. Fix any index $i \in\{1, \ldots, N\}$. It is sufficient to show that there is some $\beta_{i}>0$ s.t.

$$
\beta_{i} d\left(x^{(n)}, C_{i}\right) \leq d\left(x^{(n)}, C_{i}^{(n)}\right) \quad \text { for all large } n \text { active for } i .
$$

Case 1: $i \in\{1, \ldots, N\} \backslash I_{\partial}$. Then $\beta_{i}=\beta$ does the job for $(*)$.

Case 2: $i \in I_{\partial}$. Since $\left(x^{(n)}\right)$ is bounded, there is some $M>0$ s.t. for all $n \geq 0$, $\left\|\hat{x}-x^{(n)}\right\| \leq M$ and the norm of every subgradient of $f_{i}$ at every $x^{(n)}$ is at most $M$. Now 
fix $n$ active for $i$. Without loss, we assume that $f_{i}\left(x^{(n)}\right)>0$ (otherwise, $(*)$ holds trivially). Define

$$
\left.\epsilon:=-f_{i}(\hat{x})>0, \quad \lambda:=\frac{\epsilon}{f_{i}\left(x^{(n)}\right)+\epsilon} \in\right] 0,1[
$$

and set

$$
y:=(1-\lambda) \hat{x}+\lambda x^{(n)} .
$$

Then

$$
f_{i}(y)=f_{i}\left((1-\lambda) \hat{x}+\lambda x^{(n)}\right) \leq(1-\lambda) f_{i}(\hat{x})+\lambda f_{i}\left(x^{(n)}\right)=0
$$

hence $y \in C_{i}$. We estimate

$$
\begin{aligned}
d^{2}\left(x^{(n)}, C_{i}\right) & \leq\left\|x^{(n)}-y\right\|^{2}=(1-\lambda)^{2}\left\|\hat{x}-x^{(n)}\right\|^{2} \\
& =\left(\frac{f_{i}\left(x^{(n)}\right)}{f_{i}\left(x^{(n)}\right)+\epsilon}\right)^{2}\left\|\hat{x}-x^{(n)}\right\|^{2} \\
& \leq\left(\frac{f_{i}\left(x^{(n)}\right)}{\epsilon}\right)^{2} M^{2} \\
& =\left(\frac{d\left(x^{(n)}, C_{i}^{(n)}\right)\left\|g_{i}\left(x^{(n)}\right)\right\|}{\epsilon}\right)^{2} M^{2} \\
& \leq \frac{M^{4}}{\epsilon^{2}} d^{2}\left(x^{(n)}, C_{i}^{(n)}\right) .
\end{aligned}
$$

Therefore, $(*)$ holds with $\beta_{i}=\epsilon / M^{2}$ and the proof is complete.

\section{Examples.}

Censor and Lent's framework. We investigate in this subsection a framework essentially suggested by Censor and Lent [28]. They considered (cf. Example 7.14) subgradient algorithms where every index is a subgradient index; i.e., $I_{\partial}=\{1, \ldots, N\}$. Then

$$
C=\bigcap_{i=1}^{N} C_{i}=\bigcap_{i=1}^{N}\left\{x \in X: f_{i}(x) \leq 0\right\}
$$

is the set of solutions of the convex feasibility problem

$$
f_{i}(x) \leq 0 \quad \text { for } i=1, \ldots, N,
$$

where each $f_{i}$ is a continuous convex function from $X$ to $\mathbb{R}$.

THEOREM 7.13 (Censor and Lent's framework in Euclidean spaces). Suppose $X$ is finite dimensional. Then the sequence $\left(x^{(n)}\right)$ converges in norm to some point in $C$ whenever one of the following conditions holds.

(i) (random control) $\underline{\lim }_{n: n \text { active for } i} \mu_{i}^{(n)}>0$ for every index $i$.

(ii) (intermittent control) The subgradient algorithm is p-intermittent and $\sum_{n} v_{n}=$ $+\infty$ (where $v_{n}$ is defined as in Theorem 3.20.(ii)).

Proof. By Theorem 7.7 and Corollary 7.9, the subgradient algorithm is focusing. Now (i) follows from Corollary 3.12, whereas (ii) is immediate from Corollary 3.25.

We now obtain Censor and Lent's fundamental result as a special case of the last theorem. 
EXAMPLE 7.14 (Censor and Lent's [28, Thm. 1]). Suppose $X$ is finite dimensional and the subgradient algorithm is almost cyclic. Suppose further there is some $\epsilon>0$ s.t. $\epsilon \leq \alpha_{i}^{(n)} \leq$ $2-\epsilon$ for all $n$ and every index $i$ active at $n$. Then the sequence $\left(x^{(n)}\right)$ converges in norm to some point in $C$.

THEOREM 7.15 (Censor and Lent's framework in Hilbert spaces). Suppose the projection algorithm is p-intermittent and the functions $f_{i}$ have nonempty and uniformly bounded subdifferentials on bounded sets.

(i) If $\varliminf_{n: n \text { active for } i} \mu_{i}^{(n)}>0$ for every index $i$, then the sequence $\left(x^{(n)}\right)$ converges weakly to some point in $C$.

(ii) If $\sum_{n} v_{n}=+\infty$ (where $v_{n}$ is defined as in Theorem 3.20.(ii)), then the sequence $\left(x^{(n)}\right)$ has a (unique) weak cluster point in $C$.

Proof. By Theorem 7.7, the subgradient algorithm is focusing. The result is now immediate from Theorem 3.20.

EXAMPLE 7.16 (Eremin's [52, Thm. 1.1 for convex functions and subgradients]). Suppose $N=1$ and $f_{1}$ has nonempty and uniformly bounded subdifferentials on bounded sets.

(i) If there is some $\epsilon>0$ s.t. $\epsilon \leq \alpha_{1}^{(n)} \leq 1$, then the sequence $\left(x^{(n)}\right)$ converges weakly to some point in $C$.

(ii) If $\varlimsup_{n} \alpha_{1}^{(n)}<2$ and $\sum_{n} \alpha_{1}^{(n)}=+\infty$, then the sequence $\left(x^{(n)}\right)$ converges weakly to some point in $C$.

\section{REMARKS 7.17.}

- Eremin considered a more abstract iteration scheme.

- In view of Theorem 7.15, the assumptions in Example 7.16 can be weakened to "there is some $\epsilon>0$ s.t. $\epsilon \leq \alpha_{1}^{(n)} \leq 2-\epsilon$ " for (i), and " $\sum_{n} \alpha_{1}^{(n)}\left(2-\alpha_{1}^{(n)}\right)=+\infty$ " for (ii).

THEOREM 7.18 (Censor and Lent's framework with a Slater point). Suppose each function $f_{i}$ has nonempty and uniformly bounded subdifferentials on bounded sets and there is some Slater point $\hat{x} \in C$, i.e., $f_{i}(\hat{x})<0$, for every index $i$. Then the sequence $\left(x^{(n)}\right)$ converges in norm to some point in $X$, say $x$.

(i) If $\sum_{n} \mu_{i}^{(n)}=+\infty$ for every index $i$, then $x \in C$.

(ii) If the subgradient algorithm is intermittent and there is some $\epsilon>0$ s.t. $\epsilon \leq \alpha_{i}^{(n)} \leq$ $2-\epsilon$ and $\epsilon \leq \lambda_{i}^{(n)}$ for all large $n$ and every index $i$ active at $n$, then $x \in C$ and the sequence $\left(x^{(n)}\right)$ converges linearly to $x$.

Proof. By Theorem 7.12, the subgradient algorithm is linearly focusing. The Slater point $\hat{x}$ lies in the interior of $C_{i}=\left\{x \in X: f_{i}(x) \leq 0\right\}$; thus $\hat{x} \in$ int $C$ and $\left(C_{1}, \ldots, C_{N}\right)$ is boundedly linearly regular (Corollary 5.14). (i) follows from Theorem 3.20.(iii), whereas (ii) follows from Theorem 5.7.

EXAMPLE 7.19 (De Pierro and Iusem's [40, Thm. 2]). Suppose $X$ is finite dimensional and there is some Slater point $\hat{x} \in C$. Suppose further the subgradient algorithm is almost cyclic and there is some $\epsilon>0$ s.t. $\epsilon \leq \alpha_{i}^{(n)} \leq 2-\epsilon$ for all $n$ and every index $i$ active at $n$. Then the sequence $\left(x^{(n)}\right)$ converges linearly to some point in $C$.

Proof. Combine Corollary 7.9 and Theorem 7.18.(ii).

REMARK 7.20. De Pierro and Iusem's proof is different from ours. They obtain Example 7.19 via an investigation of an iteration that converges finitely when a Slater point exists (but may diverge otherwise).

EXAMPLE 7.21 (Eremin's [52, Thm. 1.3]). Suppose each function $f_{i}$ has nonempty and uniformly bounded subdifferentials on bounded sets and there is some Slater point $\hat{x} \in C$. If $0<\lambda_{i}^{(n)} \equiv \lambda_{i}$ and $0<\alpha_{i}^{(n)} \equiv \alpha_{i}<2$ for every index $i$, then the sequence $\left(x^{(n)}\right)$ converges linearly to some point in $C$.

Proof. The subgradient algorithm is weighted; hence it is 1-intermittent and Theorem 7.18.(ii) applies. 
Polyak's framework. In this subsection, we concentrate on a framework suggested by Polyak [86]. He considered a subgradient algorithm where

$$
N=2, \quad I_{\partial}=\{1\}, \text { and } C_{2}^{(n)} \equiv C_{2} .
$$

Hence the set $C=C_{1} \cap C_{2}=\left\{x \in C_{2}: f_{1}(x) \leq 0\right\}$ describes the solutions of the convex feasibility problem

$$
f_{1}(x) \leq 0, \quad x \in C_{2},
$$

where $f_{1}$ is a continuous convex function from $X$ to $\mathbb{R}$.

We can view Polyak's framework as a special case of Censor and Lent's framework by setting $N=2$ and letting $f_{2}=d\left(\cdot, C_{2}\right)$. We now "translate" some results obtained in the last subsection to this framework.

For example, Theorem 7.15.(i) becomes the following theorem.

THEOREM 7.22. Suppose the projection algorithm is 2 -intermittent and the function $f_{1}$ has nonempty and uniformly bounded subdifferentials on bounded sets. If $\underline{\lim }_{n: n \text { active for } i} \mu_{i}^{(n)}>0$ for $i=1$ and 2 , then the sequence $\left(x^{(n)}\right)$ converges weakly to some point in $C$.

EXAMPLE 7.23 (Polyak's [86, Thm. 1]). Suppose the function $f_{1}$ has nonempty and uniformly bounded subdifferentials on bounded sets. If the subgradient algorithm is cyclic and there is some $\epsilon>0$ s.t. $\epsilon \leq \alpha_{1}^{(n)} \leq 2-\epsilon, \alpha_{2}^{(n)} \equiv 1$ for all $n$, then the sequence $\left(x^{(n)}\right)$ converges weakly to some point in $C$.

A "translation" of Theorem 7.13.(ii) yields the following theorem.

THEOREM 7.24. Suppose $X$ is finite dimensional. If the subgradient algorithm is 2intermittent and $\sum_{n} v_{n}=+\infty$ (where $v_{n}$ is defined as in Theorem 3.20.(ii)), then the sequence $\left(x^{(n)}\right)$ converges in norm to some point in $C$.

EXAMPLE 7.25 (a special case of Allen et al.'s [4, Prop. 7]). Suppose $X$ is finite dimensional, the subgradient algorithm is cyclic, and there is some $\epsilon>0$ s.t. $0<\alpha_{1}^{2 n} \leq 2-\epsilon$ and $\alpha_{2}^{(n)} \equiv \alpha_{1}^{(2 n+1)} \equiv 1$ for all $n \geq 0$. If $\sum_{n} \alpha_{1}^{2 n}=+\infty$, then the sequence $\left(x^{(n)}\right)$ converges in norm to some point in $C$.

Proof. The subgradient algorithm is certainly 2-intermittent; hence define $v_{n}$ as in Theorem 3.20.(ii) and check that $v_{n}=\alpha_{1}^{(2 n)}\left(2-\alpha_{1}^{(2 n)}\right) \geq \alpha_{1}^{(2 n)} \epsilon$. Therefore, $\sum_{n} v_{n}=+\infty$ and the result follows from Theorem 7.24.

REMARKS 7.26.

- An inspection of the proof shows that we can replace the assumptions on $\left(\alpha_{1}^{(2 n)}\right)$ by the more general " $\sum_{n} \alpha_{1}^{(2 n)}\left(2-\alpha_{1}^{(2 n)}\right)=+\infty$."

- Allen et al. [4] also investigated the situation where it is allowed that $f_{1}$ takes the value $+\infty$ and $C$ is empty.

The next theorem does not follow from Censor and Lent's framework. The necessary work, however, is modest.

THEOREM 7.27. Suppose the subgradient algorithm is intermittent and there is some $\hat{x} \in$ $C_{2}$ with $f_{1}(\hat{x})<0$. Suppose further $f_{1}$ has nonempty and uniformly bounded subdifferentials on bounded sets. If there is some $\epsilon>0$ s.t. $\epsilon \leq \alpha_{i}^{(n)} \leq 2-\epsilon$ and $\epsilon \leq \lambda_{i}^{(n)}$ for all large $n$ and every index $i$ active at $n$, then the sequence $\left(x^{(n)}\right)$ converges linearly to some point in $C$.

Proof. By Theorem 7.12, the subgradient algorithm is linearly focusing. Since $\hat{x} \in$ $C_{2} \cap$ int $C_{1}$, the pair $\left(C_{1}, C_{2}\right)$ is boundedly linearly regular (Corollary 5.14). Now apply Theorem 5.7.

EXAMPLE 7.28 (a case of Polyak's [86, Thm. 4]). Suppose the subgradient algorithm is cyclic and there is some $\hat{x} \in C_{2}$ with $f_{1}(\hat{x})<0$. Suppose further $f_{1}$ has nonempty and uniformly bounded subdifferentials on bounded sets. If there is some $\epsilon>0$ s.t. $\epsilon \leq \alpha_{1}^{(2 n)} \leq$ 
$2-\epsilon$ and $\alpha_{1}^{(2 n+1)} \equiv \alpha_{2}^{(n)} \equiv 1$ for all $n$, then the sequence $\left(x^{(n)}\right)$ converges linearly to some point in $C$.

REMARKS 7.29. On first sight, Polyak's framework looks fairly special since it deals only with one function. A standard trick, however, allows one to handle finitely many convex functions. Suppose we are given $M$ continuous convex functions $\phi_{1}, \ldots, \phi_{M}$ from $X$ to $\mathbb{R}$. If we want to solve the convex feasibility problem

$$
x \in C_{2} \quad \text { and } \quad \phi_{i}(x) \leq 0, \quad i=1, \ldots, M,
$$

then we simply set

$$
f_{1}:=\max \left\{\phi_{1}, \ldots, \phi_{M}\right\}, \quad C_{1}:=\left\{x \in X: f_{1}(x) \leq 0\right\},
$$

and we see that $C=C_{1} \cap C_{2}$ are precisely the solutions of the above problem. Hence all methods discussed in this subsection are applicable. For example, the reformulation of the last theorem to this situation yields a partial generalization of Polyak's [86, Thm. 6]. It only remains to describe $\partial f_{1}$. The reader can find a formula in Ioffe and Tihomirov's book [68, Thm. 3 on p. 201f] that becomes in our setting

$$
\partial f_{1}(x)=\overline{\operatorname{conv}} \bigcup_{j: f_{1}(x)=\phi_{j}(x)} \partial \phi_{j}(x) .
$$

A generalization of Dos Santos's framework. In this section, we discuss a generalization of a framework due to Dos Santos (cf. Example 7.34). On first sight, this framework looks like a subgradient algorithm; it is, however, actually a projection algorithm as defined in $\S 4$. It works as follows.

Suppose we are given $M$ continuous convex functions $\phi_{i, k}$ from $X$ to $\mathbb{R}$ that are partitioned into $N(\leq M)$ "blocks," where the $i$ th block consists of $M_{i}$ functions

$$
\left\{\phi_{i, 1}, \ldots, \phi_{i, M_{i}}\right\} \text { for every index } i,
$$

so that $M_{1}+\cdots+M_{N}=M$. Let

$$
C_{i}=\left\{x \in X: \phi_{i, k}(x) \leq 0 \text { for } k=1, \ldots, M_{i}\right\} \text { for every index } i ;
$$

then $C=\bigcap_{i=1}^{N} C_{i}$ is the set of solutions of the convex feasibility problem

$$
\phi_{i, k}(x) \leq 0, \quad i=1, \ldots, N, \quad k=1, \ldots, M_{i} .
$$

As always, we assume feasibility; i.e., $C$ is nonempty.

Given a point $x^{(n)}$, we define $N$ continuous convex functions

$$
f_{i}^{(n)}: X \longrightarrow \mathbb{R}: x \longmapsto \sum_{k=1}^{M_{i}} \omega_{i, k}^{(n)} \frac{\phi_{i, k}^{+}\left(x^{(n)}\right)}{\left\|\psi_{i, k}\left(x^{(n)}\right)\right\|^{2}} \phi_{i, k}^{+}(x),
$$

where we use the convention that

$$
\frac{0}{0}:=0
$$

and where $\psi_{i, k}\left(x^{(n)}\right) \in \partial \phi_{i, k}^{+}\left(x^{(n)}\right)$ and $\omega_{i, k}^{(n)}$ are nonnegative real numbers with $\sum_{k=1}^{M_{i}} \omega_{i, k}^{(n)}=1$ for every index $i$ and $k=1, \ldots, M_{i}$. We further set

$$
g_{i}^{(n)}\left(x^{(n)}\right):=\sum_{k=1}^{M_{i}} \omega_{i, k}^{(n)} \frac{\phi_{i, k}^{+}\left(x^{(n)}\right)}{\left\|\psi_{i, k}\left(x^{(n)}\right)\right\|^{2}} \psi_{i, k}\left(x^{(n)}\right) \quad \text { for every index } i
$$


and note that $g_{i}^{(n)}\left(x^{(n)}\right) \in \partial f_{i}^{(n)}\left(x^{(n)}\right)$. Sticking to the notation of Lemma 7.3, we finally define $N$ closed convex sets by

$$
C_{i}^{(n)}:=H\left(f_{i}^{(n)}, x^{(n)}, g_{i}^{(n)}\left(x^{(n)}\right)\right) \quad \text { for every index } i .
$$

Then Lemma 7.3 (and the convention $\frac{0}{0}:=0$ ) yields the following lemma.

LEMMA 7.30. For every index $i$ and all $n \geq 0$,

$$
C_{i}^{(n)} \supseteq\left\{x \in X: f_{i}^{(n)}(x) \leq 0\right\}
$$

$$
\begin{aligned}
& \supseteq \bigcap_{k=1}^{M_{i}}\left\{x \in X: \phi_{i, k}(x) \leq 0\right\} \\
& =C_{i} .
\end{aligned}
$$

(ii) $P_{i}^{(n)} x^{(n)}=x^{(n)}-\left(\frac{f_{i}^{(n)}\left(x^{(n)}\right)}{\left\|g_{i}^{(n)}\left(x^{(n)}\right)\right\|^{2}}\right) g_{i}^{(n)}\left(x^{(n)}\right)$.

(iii) $d\left(x^{(n)}, C_{i}^{(n)}\right)=\frac{f_{i}^{(n)}\left(x^{(n)}\right)}{\left\|g_{i}^{(n)}\left(x^{(n)}\right)\right\|}$.

By Lemma 7.30.(i), we are thus given a projection algorithm to which we refer as the generalized DS algorithm. Dos Santos $[47, \S 6]$ gave an excellent motivation for a special case of the generalized DS algorithm. Of course, now we wish to bring our convergence results into play; hence, we must know what makes a generalized DS algorithm (linearly) focusing.

DEFINITION 7.31 (control). We say that the generalized DS algorithm considers most violated constraints if there is some $\tau>0$ s.t. for every index $i$, there is some $k \in\left\{1, \ldots, M_{i}\right\}$ with

$$
\phi_{i, k}^{+}\left(x^{(n)}\right)=\max _{l} \phi_{i, l}^{+}\left(x^{(n)}\right) \text { and } \omega_{i, k}^{(n)} \geq \tau \text { for all } n \geq 0 .
$$

THEOREM 7.32 (prototype of a (linearly) focusing generalized DS algorithm). Suppose the generalized DS algorithm considers most violated constraints and the functions $\phi_{i, k}$ have nonempty and uniformly bounded subdifferentials on bounded sets.

(i) Then the generalized DS algorithm is focusing.

(ii) Suppose that in addition for every index $i$ at least one of the following conditions holds.

1. There is some Slater point $\hat{x}_{i} \in C_{i}$ s.t.

$$
\phi_{i, k}\left(\hat{x}_{i}\right)<0 \quad \text { for every } k=1, \ldots, M_{i} .
$$

2. Each $\phi_{i, k}$ is a distance function to some closed convex set $C_{i, k}$ and the $M_{i}$-tuple

$$
\left(C_{i, 1}, \ldots, C_{i, M_{i}}\right) \text { is boundedly linearly regular. }
$$

Then the generalized DS algorithm is linearly focusing.

Proof. First, we get $L_{1}>0$ s.t. $\left\|\psi_{i, k}\left(x^{(n)}\right)\right\| \leq L_{1}$ for all $n \geq 0$, every index $i$, and all $k=1, \ldots, M_{i}$. Second, we get $\tau>0$ s.t. for all $n \geq 0$ and every index $i, \omega_{i, k^{*}}^{(n)} \geq \tau$ for some $k^{*} \in\left\{1, \ldots, M_{i}\right\}$ with $\phi_{i, k^{*}}^{+}\left(x^{(n)}\right)=\max _{k} \phi_{i, k}^{+}\left(x^{(n)}\right)$. Now fix an index $i$ and $n \geq 0$ and assume (without loss, as we will see) that $x^{(n)} \notin C$. It is convenient to abbreviate

$$
\omega_{k}:=\omega_{i, k}^{(n)}, \quad q_{k}:=\frac{\phi_{i, k}^{+}\left(x^{(n)}\right)}{\left\|\psi_{i, k}\left(x^{(n)}\right)\right\|}, \quad z_{k}:=\frac{\psi_{i, k}\left(x^{(n)}\right)}{\left\|\psi_{i, k}\left(x^{(n)}\right)\right\|}
$$

and to let any appearing $k$ 's range in $\left\{k \in\left\{1, \ldots, M_{i}\right\}: z_{k} \neq 0\right\}$. Using the convexity of $\|\cdot\|$ and $(\cdot)^{2}$, we estimate 


$$
\begin{aligned}
d\left(x^{(n)}, C_{i}^{(n)}\right) & =\frac{f_{i}^{(n)}\left(x^{(n)}\right)}{\left\|g_{i}^{(n)}\left(x^{(n)}\right)\right\|}=\frac{\sum_{k} \omega_{k} q_{k}^{2}}{\left\|\sum_{k} \omega_{k} q_{k} z_{k}\right\|} \\
& \geq \frac{\sum_{k} \omega_{k} q_{k}^{2}}{\sum_{k} \omega_{k} q_{k}\left\|z_{k}\right\|}=\frac{\sum_{k} \omega_{k} q_{k}^{2}}{\sum_{k} \omega_{k} q_{k}} \\
& \geq \frac{\left(\sum_{k} \omega_{k} q_{k}\right)^{2}}{\sum_{k} \omega_{k} q_{k}}=\sum_{k} \omega_{k} q_{k} .
\end{aligned}
$$

By choice of $\tau$ and $L_{1}$, we conclude that

$$
d\left(x^{(n)}, C_{i}^{(n)}\right) \geq \frac{\tau}{L_{1}} \max _{k} \phi_{i, k}^{+}\left(x^{(n)}\right) \quad \text { for every index } i \text { and all } n \geq 0 .
$$

Note that if $x^{(n)} \in C$, then (*) holds trivially.

(i): If $\left(x^{\left(n^{\prime}\right)}\right)$ is a weakly convergent subsequence of $\left(x^{(n)}\right)$ with weak limit $x$ and $d\left(x^{\left(n^{\prime}\right)}, C_{i}^{\left(n^{\prime}\right)}\right) \longrightarrow 0$, then, by $(*), \varlimsup_{n^{\prime}} \max _{k} \phi_{i, k}^{+}\left(x^{\left(n^{\prime}\right)}\right) \leq 0$. Since $\phi_{i, k}^{+}$is weakly lower semicontinuous, this implies $\phi_{i, k}^{+}(x) \leq 0$ for $k=1, \ldots, M_{i}$. Hence $x \in C_{i}$ and the generalized DS algorithm is focusing.

(ii): Case 1: Condition 1 holds. Get $L_{2}>0$ s.t. $\left\|\hat{x}_{i}-x^{(n)}\right\| \leq L_{2}$ for all $n \geq 0$. Now fix an index $i$ and $n$. Define

$$
y:=(1-\lambda) \hat{x}+\lambda x^{(n)}
$$

where

$$
\left.\left.\lambda:=\frac{\min _{k}\left\{-\phi_{i, k}(\hat{x})\right\}}{\min _{k}\left\{-\phi_{i, k}(\hat{x})\right\}+\max _{k}\left\{\phi_{i, k}^{+}\left(x^{(n)}\right)\right\}} \in\right] 0,1\right] .
$$

Then one readily verifies that $\phi_{i, k}(y) \leq 0$ for all $k$; thus $y \in C_{i}$. We estimate

$$
\begin{aligned}
d^{2}\left(x^{(n)}, C_{i}\right) & \leq\left\|x^{(n)}-y\right\|^{2}=(1-\lambda)^{2}\left\|\hat{x}-x^{(n)}\right\|^{2} \\
& \leq\left(\frac{\max _{k}\left\{\phi_{i, k}^{+}\left(x^{(n)}\right)\right\}}{\min _{k}\left\{-\phi_{i, k}(\hat{x})\right\}}\right)^{2} L_{2}^{2} .
\end{aligned}
$$

Combining the previous estimate with $(*)$ yields

$$
\frac{\tau \min _{k}\left\{-\phi_{i, k}(\hat{x})\right\}}{L_{1} L_{2}} d\left(x^{(n)}, C_{i}\right) \leq d\left(x^{(n)}, C_{i}^{(n)}\right) \quad \text { for all } n \geq 0 .
$$

Case 2: Condition 2 holds. Because $\left(C_{i, 1}, \ldots, C_{i, M_{i}}\right)$ is boundedly linearly regular, there exists some $L_{3}>0$ s.t.

$$
d\left(x^{(n)}, C_{i}\right) \leq L_{3} \max _{k} d\left(x^{(n)}, C_{i, k}\right)=L_{3} \max _{k} \phi_{i, k}\left(x^{(n)}\right) \text { for all } n \geq 0 .
$$

Fix an index $i$. Combining the last estimate with (*) this time yields

$$
\frac{\tau}{L_{1} L_{3}} d\left(x^{(n)}, C_{i}\right) \leq d\left(x^{(n)}, C_{i}^{(n)}\right) \text { for all } n \geq 0 .
$$

In both cases, we have found an inequality that makes the generalized DS algorithm linearly focusing.

Having identified nice classes of (linearly) focusing generalized DS algorithms, we could now systematically "translate" our previous results to this situation; again, we opt for a small selection. 
Dos Santos's original framework. Dos Santos considered the situation when $N=2$, and we set $L:=M_{1}$,

$$
\phi_{1}:=\phi_{1,1}, \ldots, \phi_{L}:=\phi_{1, M_{1}},
$$

$M_{2}=1$, and $\phi_{2,1}=d\left(\cdot, C_{2}\right)$ for some closed convex nonempty set $C_{2}$. Thus $C=C_{1} \cap C_{2}$ is the set of solutions of the convex feasibility problem

$$
x \in C_{2} \quad \text { and } \quad \phi_{k}(x) \leq 0 \text { for } k=1, \ldots, L .
$$

We refer to the generalized DS algorithm in this framework as the DS algorithm.

THEOREM 7.33 (Dos Santos's original framework). Suppose the DS algorithm is intermittent and considers most violated constraints and there is some $\epsilon>0$ s.t. $\epsilon \leq \alpha_{i}^{(n)} \leq 2-\epsilon$ and $\epsilon \leq \lambda_{i}^{(n)}$ for all $n$ and every index $i$ active at $n$. Suppose further each $\phi_{k}$ has nonempty and uniformly bounded subdifferentials on bounded sets.

(i) Then the sequence $\left(x^{(n)}\right)$ converges weakly to some point in $C$. Consequently, if $X$ is finite dimensional, then the sequence $\left(x^{(n)}\right)$ converges in norm to some point in $C$.

(ii) If there is some $\hat{x} \in C_{2}$ s.t. $\phi_{k}(\hat{x})<0$ for $k=1, \ldots, L$, then the sequence $\left(x^{(n)}\right)$ converges linearly to some point in $C$.

Proof. (i): By Theorem 7.32.(i), the DS algorithm is focusing. The result now follows from Remark 3.13 and Theorem 3.20.(i).

(ii): On the one hand, the DS algorithm is linearly focusing (Theorem 7.32.(ii)). On the other hand, $\hat{x} \in C_{2} \cap$ int $C_{1}$, so $\left(C_{1}, C_{2}\right)$ is boundedly linearly regular (Corollary 5.14). Altogether (Theorem 5.7), the sequence $\left(x^{(n)}\right)$ converges linearly to some point in $C$.

EXAMPLE 7.34 (Dos Santos's [47, Thm.]). Suppose $X$ is finite dimensional, the DS algorithm is cyclic, and there is some $\epsilon>0$ s.t. $\epsilon \leq \alpha_{1}^{(2 n)} \leq 2-\epsilon$ and $\alpha_{2}^{(n)} \equiv 1$ for all $n \geq 0$. Suppose further $0<\omega_{1, k}^{(2 n)} \equiv \omega_{k}$ for $k=1, \ldots, L$. Then the sequence $\left(x^{(n)}\right)$ converges in norm to some point in $C$.

Proof. Since $\omega_{k}>0$ for all $k$, the DS algorithm certainly considers most violated constraints. Now combine Corollary 7.9 and Theorem 7.33.(i).

REMARKS 7.35.

- For $L=1$, Dos Santos's and Polyak's frameworks coincide.

- Dos Santos reports good numerical results on his algorithm. Theorem 7.33.(ii) shows that the qualitative performances of his and Censor and Lent's frameworks are comparable (cf. Theorem 7.18 and Example 7.19).

The polyhedral framework. The polyhedral framework is the special case of the generalized Dos Santos framework, where $\phi_{i, k}$ is the distance function to some polyhedron $C_{i, k}$ for every index $i$ and all $k=1, \ldots, M_{i}$. Throughout this subsection, we investigate this situation.

THEOREM 7.36 (polyhedron framework). In the polyhedral framework, suppose the generalized DS algorithm considers most violated constraints. Suppose further it is intermittent or considers remotest sets. Suppose finally there is some $\epsilon>0$ s.t. $\epsilon \leq \alpha_{i}^{(n)} \leq 2-\epsilon$ and $\epsilon \leq \lambda_{i}^{(n)}$ for all $n$ and every index $i$ active at $n$. Then the sequence $\left(x^{(n)}\right)$ converges linearly to some point in $C$ with a rate independent of the starting point.

Proof. For every index $i$, the $M_{i}$-tuple $\left(C_{i, 1}, \ldots, C_{i, M_{i}}\right)$ is linearly regular (Corollary 5.26). Hence, by Theorem 7.32.(ii), the generalized DS algorithm is linearly focusing. Now each $C_{i}$ is also a polyhedron; thus by Corollary $5.26,\left(C_{1}, \ldots, C_{N}\right)$ is linearly regular. Therefore, the result follows from Theorem 5.7 (for intermittent control) or Theorem 5.8 (if the algorithm considers remotest sets).

REMARK 7.37. If $N=M$, each $C_{i, 1}$ is a halfspace, $\omega_{i, 1}^{(n)} \equiv \omega_{i}>0$, and there is some $\epsilon>0$ s.t. $\epsilon \leq \alpha_{i}^{(n)} \equiv \alpha^{(n)} \leq 2-\epsilon$ for all $n$ and every index $i$, then we recapture Example 6.40 (which is due to De Pierro and Iusem [39]).

We register two more special cases. 
EXAMPLE 7.38 (Merzlyakov's [80, Thm.]). In the polyhedral framework, suppose $X$ is finite dimensional, $N=1$, each $C_{1, i}$ is a halfspace, and the generalized DS algorithm considers most violated constraints. Suppose further $0<\alpha_{1}^{(n)} \equiv \alpha_{1}<2$. Then the sequence $\left(x^{(n)}\right)$ converges linearly to some point in $C$ with a rate independent of the starting point.

REMARK 7.39. Merzlyakov [80] actually considered a more general version, where the $\omega_{1, i}^{(n)}$ need not necessarily sum up to 1 .

EXAMPLE 7.40 (Yang and Murty's [105]). In the polyhedral framework, suppose $X$ is finite dimensional, each $C_{i, k}$ is a halfspace, and there is some $\epsilon>0$ s.t. the generalized DS algorithm satisfies

$$
\omega_{i, k}^{(n)} \begin{cases}=0 & \text { if } x^{(n)} \in C_{i, k}, \\ \geq \epsilon & \text { otherwise }\end{cases}
$$

for all $n$, every index $i$, and all $k=1, \ldots, M_{i}$. Suppose further the relaxation parameters satisfy $0<\alpha_{i}^{(n)} \equiv \alpha<2$ for all $n$ and every index $i$. Then the sequence $\left(x^{(n)}\right)$ converges linearly to some point in $C$ with a rate independent of the starting point whenever one of the following conditions holds.

1. (basic surrogate constraint method: $[105, \S 3]) N=1$.

2. (sequential surrogate constraint method: [105, §4]) The generalized DS algorithm is cyclic.

3. (parallel surrogate constraint method: $[105, \S 5]$ ) There is some $\epsilon^{\prime}>0$ s.t. $x^{(n)} \notin C_{i}$ implies $\lambda_{i}^{(n)} \geq \epsilon^{\prime}$ for all $n$ and every index $i$.

Proof. Obviously, the generalized DS algorithm considers most violated constraints. The first condition is a special case of the second one, which in turn follows from Theorem 7.36. The assumption in the third condition guarantees that the algorithm considers remotest sets; hence, this case is also covered by Theorem 7.36.

Acknowledgments. It is our pleasure to thank Yair Censor, Frank Deutsch, Sjur Flåm, Krzysztof Kiwiel, Adrian Lewis, Alex Simonič, Levent Tunçel, Jon Vanderwerff, and two referees for helpful suggestions.

Final remarks. This attempt to review and unify is necessarily incomplete-it is impossible to keep track of all relevant manuscripts in the field. We thus wish to apologize to the contributors who should have been included here but are not. The manuscript is merely a snapshot of what the authors knew in mid-1993; time, of course, has not stood still. The manuscripts sent to us recently by Combettes [30-37], García-Palomares [55], and Kiwiel [72-75] deal with exciting new generalizations and deserve much attention. A synthesis of a selection of these results may be found in the first author's Ph.D. thesis (Projection Algorithms and Monotone Operators, Simon Fraser University, 1996).

\section{Index.}

active index, 378

active remotest index, 390

Aharoni and Censor's framework, 389

algorithm, 378

almost cyclic algorithm, 382

almost cyclic control, 382

alternating projections, 386

angle, 396

asymptotically regular algorithm, 378

asymptotically regular sequence, 374

attracting mapping, 372

averaged mapping, 370

block algorithm, 382

block control, 382

bounded linear regularity, 393

bounded regularity, 391

boundedly linearly regular, 393 
boundedly regular, 391

Butnariu and Censor's framework, 390

Censor and Elfving's framework, 408

Censor and Lent's framework, 414

Cimmino's method, 405

cone, 372

consideration of remotest sets control, 390

considers most violated constraints, 418

considers only blocks, 382

considers remotest sets, 390

converges actively pointwise, 381

converges linearly, 371

convex feasibility problem, 367

cyclic algorithm, 382

cyclic control, 382

cyclic projections, 401

demiclosedness principle, 370

distance function, 371

Dos Santos's original framework, 420

DS algorithm, 420

Fejér monotone sequence, 375

firmly nonexpansive mapping, 370

fixed points, 370

Flåm and Zowe's framework, 389

focusing algorithm, 380

focusing subgradient algorithm, 412

generalized DS algorithm, 418

halfspace, 406

has constant sets, 385

hyperplane, 403

hyperslab, 409

icr, 372

index, 377

int, 372

intermittent algorithm, 382

intermittent control, 382

intrinsic core, 372

isometry, 370

linear convergence, 371

linear regularity, 393

linearly regular, 393

linearly focusing projection algorithm, 386

linearly focusing subgradient algorithm, 413

linear feasibility problem, 406

Mosco convergence, 385 nonexpansive mapping, 370

overrelaxation, 378

parallel algorithm, 378

parallel control, 378

parallelogram law, 369

Pierra's product space formalization, 397

Polyak's framework, 416

polyhedral framework, 420

polyhedron, 372

positive cone, 410

positive part, 372

projection, 370

projection algorithm, 385

random algorithm, 382

random control, 382

random projections, 386

regular, 391

regularity, 391

relaxation parameter, 377

relaxed firmly nonexpansive mapping, 370

remotest set control, 390

sequence of active remotest indices, 390

sequence of the algorithm, 378

singular algorithm, 378

singular control, 378

Slater point, 413

span, 371

strictly nonexpansive mapping, 370

strict convexity, 369

strongly attractive mapping, 372

strongly focusing projection

algorithm, 386

subgradient algorithm, 411

subgradient index, 411

Tseng's framework, 381

underrelaxation, 378

unrelaxed algorithm, 378

unrelaxed control, 378

von Neumann's alternating projection algorithm, 386

von Neumann/Halperin's framework, 403

weight, 378

weighted algorithm, 378

weighted control, 378 


\section{REFERENCES}

[1] S. Agmon, The relaxation method for linear inequalities, Canad. J. Math., 6 (1954), pp. 382-392.

[2] R. Aharoni, A. Berman, AND Y. Censor, An interior points algorithm for the convex feasibility problem, Adv. Appl. Math., 4 (1983), pp. 479-489.

[3] R. AhARONI AND Y. CENSOR, Block-iterative projection methods for parallel computation of solutions to convex feasibility problems, Linear Algebra Appl., 120 (1989), pp. 165-175.

[4] E. Allen, R. Helgason, J. Kennington, And B. Shetty, A generalization of Polyak's convergence result for subgradient optimization, Math. Programming, 37 (1987), pp. 309-317.

[5] I. AMEMIYA AND T. ANDo, Convergence of random products of contractions in Hilbert space, Acta Sci. Math. (Szeged), 26 (1965), pp. 239-244.

[6] R. ANSORGE, Connections between the Cimmino-method and the Kaczmarz-method for the solution of singular and regular systems of equations, Computing, 33 (1984), pp. 367-375.

[7] J. B. BAIllon, Comportement Asymptotique des Contractions et Semi-Groupes de Contractions, Ph.D. thesis, University of Paris VI, 1978. (In French.)

[8] J. B. BAILLON AND H. BREZIS, Une remarque sur le comportement asymptotique des semigroupes non lineaires, Houston J. Math., 2 (1976), pp. 5-7. (In French.)

[9] J. B. BAILLON, R. E. BRUCK, AND S. REICH, On the asymptotic behaviour of nonexpansive mappings and semigroups in Banach spaces, Houston J. Math., 4 (1978), pp. 1-9.

[10] M. Baronti AND P. L. PAPINI, Convergence of sequences of sets, in Methods of Functional Analysis in Approximation Theory, C. Micchelli, D. Pai, and B. Limaye, eds., Birkhäuser, Basel, 1986, pp. 135155. Internat. Ser. Numer. Math., Vol. 76. Proceedings of the International Conference held at the Indian Institute of Technology, Bombay, December 16-20, 1985.

[11] H. H. BAUSCHKE, The approximation of fixed points of compositions of nonexpansive mappings in Hilbert space, J. Math. Anal. Appl., 1996, to appear.

[12] - A norm convergence result on random products of relaxed projections in Hilbert space, Trans. Amer. Math. Soc., 347 (1995), pp. 1365-1373.

[13] H. H. BAUSCHKE AND J. M. BoRWEIN, On the convergence of von Neumann's alternating projection algorithm for two sets, Set-Valued Anal., 1 (1993), pp. 185-212.

[14] H. H. BAuschke, J. M. BorweIn, AND A. S. LeWIS, The method of cyclic projections for closed convex sets in Hilbert space, Proc. of the Special Session on Optimization and Nonlinear Analysis, Y. Censor and S. Reich, eds., Jerusalem, 1995. Contemp. Math., to appear.

[15] J. M. BorweIn, S. REICH, AND I. SHAFrir, Krasnoselski-Mann iterations in normed spaces, Canad. Math. Bull., 35 (1992), pp. 21-28.

[16] L. M. BREgman, The method of successive projection for finding a common point of convex sets, Soviet Math. Dokl., 6 (1965), pp. 688-692.

[17] F. E. BROWDER, Convergence theorems for sequences of nonlinear operators in Banach spaces, Math. Z., 100 (1967), pp. 201-225.

[18] R. E. BRUCK, Random products of contractions in metric and Banach spaces, J. Math. Anal. Appl., 88 (1982), pp. 319-332.

[19] R. E. BRUCK AND S. REICH, Nonexpansive projections and resolvents of accretive operators in Banach spaces, Houston J. Math., 3 (1977), pp. 459-470.

[20] D. BUTNARIU AND Y. CENSOR, On the behavior of a block-iterative projection method for solving convex feasibility problems, J. Comput. Math., 32 (1990), pp. 79-94.

[21] Y. CENSOR, Row-action methods for huge and sparse systems and their applications, SIAM Rev., 23 (1981), pp. 444-466.

[22] - Iterative methods for the convex feasibility problem, in Convexity and Graph Theory, M. Rosenfeld and J. Zaks, eds., North-Holland, Amsterdam, 1984, pp. 83-91. Proceedings of the Conference on Convexity and Graph Theory, Israel, March 1981. Ann. Discrete Math., Vol. 20.

[23] - Parallel application of block-iterative methods in medical imaging and radiation therapy, Math. Programming, 42 (1988), pp. 307-325.

[24] - On variable block algebraic reconstruction techniques, in Mathematical Methods in Tomography, G. Herman, A. Louis, and F. Natterer, eds., Springer, New York, 1990, pp. 133-140. Proceedings of a Conference held in Oberwolfach, Germany, June 11-15. Lecture Notes in Math., Vol. 1497.

[25] Y. CEnsor, M. D. Altschuler, AND W. D. Powlis, On the use of Cimmino's simultaneous projections method for computing a solution of the inverse problem in radiation therapy treatment planning, Inverse Problems, 4 (1988), pp. 607-623.

[26] Y. Censor AND T. Elfving, New methods for linear inequalities, Linear Algebra Appl., 42 (1982), pp. 199-211.

[27] Y. CENSOR AND G. T. HERMAN, On some optimization techniques in image reconstruction from projections, Appl. Numer. Math., 3 (1987), pp. 365-391.

[28] Y. CENSOR AND A. Lent, Cyclic subgradient projections, Math. Programming, 24 (1982), pp. 233-235. 
[29] G. Cimmino, Calcolo approssimate per le soluzioni dei sistemi di equazioni lineari, La Ricerca scientifica ed il Progresso tecnico nell' Economia nazionale (Roma), 9 (1938), pp. 326-333. Consiglio Nazionale delle Ricerche. Ministero dell' Educazione nazionale.

[30] P. L. CombetTes, The foundations of set theoretic estimation, Proc. IEEE, 81 (1993), pp. 182-208.

[31] - Convex set theoretic image recovery by extrapolated iterations of parallel subgradient projections, IEEE Trans. on Image Processing, 1996, to appear.

[32] - Inconsistent signal feasibility problems: Least-squares solutions in a product space, IEEE Trans. Signal Processing, 42 (1994), pp. 2955-2966.

[33] - Construction d'un point fixe commun à une famille de contractions fermes, C. R. Acad. Sci. Paris Sér. I Math., 320 (1995), pp. 1385-1390.

[34] - The Convex Feasibility Problem in Image Recovery, Vol. 95 of Advances in Imaging and Electron Physics, Academic Press, New York, 1995.

[35] - Hilbertian convex feasibility problem: Convergence of projection methods, Appl. Math. Optim., 1996, to appear.

[36] P. L. COMBETTES AND H. PuH, Extrapolated projection method for the euclidean convex feasibility problem, Tech. report, City University of New York, 1993.

[37] - Iterations of parallel convex projections in Hilbert space, Numer. Funct. Anal. Optim., 15 (1994), pp. 225-243.

[38] G. Crombez, Image recovery by convex combinations of projections, J. Math. Anal. Appl., 155 (1991), pp. 413-419.

[39] A. R. DE PIERro AND A. N. IUSEM, A simultaneous projections method for linear inequalities, Linear Algebra Appl., 64 (1985), pp. 243-253.

[40] - A finitely convergent "row-action" method for the convex feasibility problem, Appl. Math. Optim., 17 (1988), pp. 225-235.

[41] - On the asymptotic behaviour of some alternate smoothing series expansion iterative methods, Linear Algebra Appl., 130 (1990), pp. 3-24.

[42] F. DEUTSCH, Rate of convergence of the method of alternating projections, in Parametric Optimization and Approximation, B. Brosowski and F. Deutsch, eds., Birkhäuser, Basel, 1983, pp. 96-107. Internat. Ser. Numer. Math., Vol. 76.

[43] The angle between subspaces of a Hilbert space, in Approximation Theory, Wavelets and Applications, S. P. Singh, ed., Kluwer Academic, 1995, pp. 107-130.

[44] - The method of alternating orthogonal projections, in Approximation Theory, Spline Functions and Applications, S. Singh, ed., Kluwer Academic, 1992, pp. 105-121. Proceedings of a Conference held in the Hotel villa del Mare, Maratea, Italy between April 28, 1991 and May 9, 1991.

[45] F. DEUTSCH AND H. HUNDAL, The rate of convergence for the method of alternating projections II, J. Math. Anal. Appl., to appear.

[46] B. DJAFARI RouHANI, Asymptotic behaviour of almost nonexpansive sequences in a Hilbert space, J. Math. Anal. Appl., 151 (1990), pp. 226-235.

[47] L. T. Dos SANTOS, A parallel subgradient method for the convex feasibility problem, J. Comput. Appl. Math., 18 (1987), pp. 307-320.

[48] J. M. DYE AND S. REICH, Unrestricted iterations of nonexpansive mappings in Hilbert space, Nonlinear Anal., 18 (1992), pp. 199-207.

[49] P. P. EgGermont, G. T. HERMAN, and A. LENT, Iterative algorithms for large partitioned linear systems, with applications to image reconstruction, Linear Algebra Appl., 40 (1981), pp. 37-67.

[50] I. Ekeland And R. Temam, Convex Analysis and Variational Problems, North-Holland, Amsterdam, 1976.

[51] L. ElSNER, I. KOLTRACHT, AND M. NEUMANN, Convergence of sequential and asynchronous nonlinear paracontractions, Numer. Math., 62 (1992), pp. 305-319.

[52] I. I. EREMIN, Fejér mappings and convex programming, Siberian Math. J., 10 (1969), pp. 762-772.

[53] S. D. FLÅM AND J. ZowE, Relaxed outer projections, weighted averages and convex feasibility, BIT, 30 (1990), pp. 289-300.

[54] K. FRIEDRICHS, On certain inequalities and characteristic value problems for analytic functions and for functions of two variables, Trans. Amer. Math. Soc., 41 (1937), pp. 321-364.

[55] U. García-Palomares, Parallel projected aggregation methods for solving the convex feasibility problem, SIAM J. Optim., 3 (1993), pp. 882-900.

[56] A. GENEL AND J. LindEnSTRAuSs, An example concerning fixed points, Israel J. Math., 22 (1975), pp. 81-86.

[57] K. GoEBEL AND W. A. KIRK, Iteration processes for nonexpansive mappings, in Topological Methods in Nonlinear Functional Analysis, S. Singh, S. Thomeier, and B. Watson, eds., American Mathematical Society, 1983, pp. 115-123. Contemp. Math., Vol. 21.

[58] - Topics in Metric Fixed Point Theory, Cambridge University Press, 1990. Cambridge Stud. Adv. Math., Vol. 28.

[59] R. GORDON, R. BENDER, AND G. T. HERMAN, Algebraic reconstruction techniques (ART) for three-dimensional electron microscopy and X-ray photography, J. Theoret. Biol., 29 (1970), pp. 471-481. 
[60] L. G. GUBIN, B. T. POLYAK, AND E. V. RAIK, The method of projections for finding the common point of convex sets, Comput. Math. Math. Phys., 7(6) (1967), pp. 1-24.

[61] I. HALPERIN, The product of projection operators, Acta Sci. Math. (Szeged), 23 (1962), pp. 96-99.

[62] B. HALPERN, Fixed points of nonexpanding maps, Bull. Amer. Math. Soc., 73 (1967), pp. 957-961.

[63] G. T. Herman, Image Reconstruction from Projections, Academic Press, New York, 1980.

[64] G. T. Herman, A. Lent, AND P. H. LuTZ, Relaxation methods for image reconstruction, Comm. ACM, 21 (1978), pp. 152-158.

[65] G. T. Herman, H. Levkowitz, H. K. Tuy, And S. MCCormick, Multilevel image reconstruction, in Multiresolution Image Processing and Analysis, A. Rosenfeld, ed., Springer, New York, 1984, pp. 121-135. Based on a workshop held in Leesburg, VA, July 19-21, 1982. Springer Ser. Inform. Sci., Vol. 12.

[66] A. J. HoffmAn, On approximate solutions of systems of linear inequalities, J. Research of the National Bureau of Standards, 49 (1952), pp. 263-265.

[67] R. B. Holmes, Geometric Functional Analysis and its Applications, Springer-Verlag, New York, 1975.

[68] A. D. Ioffe And V. M. Tinomirov, Theory of Extremal Problems, North-Holland, Amsterdam, 1979. Stud. Math. Appl., Vol. 6.

[69] V. I. IsTRATESCU, Fixed Point Theory, D. Reidel, Boston, MA, 1981.

[70] G. J. JAMESON, Topology and Normed Spaces, Chapman and Hall, London, UK, 1974.

[71] S. Kaczmarz, Angenäherte Aufösung von Systemen linearer Gleichungen, Bulletin internationel de l'Académie Polonaise des Sciences et des Lettres. Classe des Sciences mathématiques et naturelles. Séries A: Sciences mathématiques, (1937), pp. 355-357. Cracovie, Imprimerie de l'Université.

[72] K. C. KIWIEL, Block-iterative surrogate projection methods for convex feasibility problems, Linear Algebra Appl., 215 (1995), pp. 225-260.

[73] — - The efficiency of subgradient projection methods for convex optimization, part I: General level methods, SIAM J. Control Optim., 34 (1996), pp. 660-676.

[74] - The efficiency of subgradient projection methods for convex optimization, part II: Implementations and extensions, SIAM J. Control Optim., 34 (1996), pp. 677-697.

[75] K. C. KIWIEL AND B. LOPUCH, Surrogate projection methods for finding fixed points of firmly nonexpansive mappings, SIAM J. Optim., to appear.

[76] E. Klein AND A. C. Thompson, Theory of Correspondences, Wiley-Interscience, New York, 1984.

[77] P.-L. Lions, Approximation de points fixes de contractions, Comptes Rendus des Séances de l'Académie des Sciences, Séries A-B, Paris, 284 (1977), pp. 1357-1359.

[78] J. MANDEL, Convergence of the cyclic relaxation method for linear inequalities, Math. Programming, 30 (1984), pp. 218-228.

[79] S. MARUSTER, The solution by iteration of nonlinear equations in Hilbert spaces, Proc. Amer. Math. Soc., 63 (1977), pp. 69-73.

[80] Y. Merzlyakov, On a relaxation method of solving systems of linear inequalities, Comput. Math. Math. Phys., 2 (1963), pp. 504-510.

[81] J. J. MOREAU, Un cas des convergence des iterées d'une contraction d'un éspace hilbertien, Comptes Rendus des Séances de l'Académie des Sciences, Séries A-B, Paris, 286 (1978), pp. 143-144. (In French.)

[82] U. Mosco, Convergence of convex sets and of solutions of variational inequalities, Adv. Math., 3 (1969), pp. 510-585.

[83] T. S. MOTZKIn AND I. J. SchoENBERG, The relaxation method for linear inequalities, Canad. J. Math., 6(1954), pp. 393-404.

[84] Z. OPIAL, Weak convergence of the sequence of successive approximations for nonexpansive mappings, Bull. Amer. Math. Soc., 73 (1967), pp. 591-597.

[85] G. PIERRA, Decomposition through formalization in a product space, Math. Programming, 28 (1984), pp. 96-115.

[86] B. T. PolyaK, Minimization of unsmooth functionals, Comput. Math. Math. Phys., 9 (1969), pp. 14-29.

[87] H. RÅDSTRÖM, An embedding theorem for spaces of convex sets, Proc. Amer. Math. Soc., 3 (1952), pp. 165-169.

[88] A. W. Roberts and D. E. Varberg, Convex Functions, Academic Press, New York, 1973.

[89] D. SCHOTT, A general iterative scheme with applications to convex optimization and related fields, Optimizaton, 22 (1991), pp. 885-902.

[90] H. F. Senter AND W. G. Dotson, JR., Approximating fixed points of nonexpansive mappings, Proc. Amer. Math. Soc., 44 (1974), pp. 375-380.

[91] M. I. SEZAN, An overview of convex projections theory and its applications to image recovery problems, Ultramicroscopy, 40 (1992), pp. 55-67.

[92] N. Z. SHOR, Minimization Methods for Non-Differentiable Functions, Springer-Verlag, Berlin, 1985. Springer Ser. Comput. Math., Vol. 3.

[93] A. Simonič, personal communication.

[94] K. T. SMith, D. C. Solmon, AND S. L. WAGner, Practical and mathematical aspects of the problem of reconstructing objects from radiographs, Bull. Amer. Math. Soc., 83 (1977), pp. 1227-1270. 
[95] H. STARK, ED., Image Recovery: Theory and Application, Academic Press, Orlando, FL, 1987.

[96] K. TANABE, Projection method for solving a linear system of linear equations and its applications, Numer. Math., 17 (1971), pp. 203-214.

[97] M. R. TRUMMER, Reconstructing pictures from projections: On the convergence of the ART algorithm with relaxation, Computing, 26 (1981), pp. 189-195.

[98] — SMART-an algorithm for reconstructing pictures from projections, Z. Angew. Math. Phys., 34 (1983), pp. 746-753.

[99] - A note on the ART of relaxation, Computing, 33 (1984), pp. 349-352.

[100] P. TSENG, On the convergence of the products of firmly nonexpansive mappings, SIAM J. Optim., 2 (1992), pp. 425-434.

[101] M. TsukADA, Convergence of best approximations in a smooth Banach space, J. Approx. Theory, 40 (1984), pp. 301-309.

[102] M. A. VIERGEVER, Introduction to discrete reconstruction methods in medical imaging, in Mathematics and Computer Science in Medical Imaging, M. Viergever and A. Todd-Pokropek, eds., Berlin, 1988, Springer-Verlag, pp. 43-65. NATO Advanced Science Institute Series F: Computer and Systems Sciences, Vol. 39. Proceedings, held in Il Ciocco, Italy, September 21-October 4, 1986.

[103] J. von NeUmann, Functional Operators, Vol. II. The Geometry of Orthogonal Spaces, Princeton University Press, Princeton, NJ, 1950. Ann. Math. Stud., Vol. 22. Reprint of mimeographed lecture notes first distributed in 1933.

[104] R. WitTMann, Approximation of fixed points of nonexpansive mappings, Arch. Math. (Basel), 58 (1992), pp. 486-491.

[105] K. YANG AND K. G. MurTY, New iterative methods for linear inequalities, J. Optim. Theory Appl., 72 (1992), pp. 163-185.

[106] D. C. Youla, Mathematical theory of image restoration by the method of convex projections, in Image Recovery: Theory and Applications, H. Stark, ed., Academic Press, Orlando, FL, 1987, pp. 29-77.

[107] - On deterministic convergence of iterations of relaxed projection operators, J. Vis. Commun. Image Rep., 1 (1990), pp. 12-20.

[108] D. C. Youla AND H. WEBB, Image reconstruction by the method of convex projections: Part 1-theory, IEEE Trans. Medical Imaging, MI-1 (1982), pp. 81-94.

[109] E. H. ZaRANTONELlo, Projections on convex sets in Hilbert space and spectral theory, in Contributions to Nonlinear Functional Analysis, E. Zarantonello, ed., Academic Press, New York, 1971, pp. 237-424. University of Wisconsin. Mathematics Research Center. Publication No. 27. 